LA-14314-MS

Approved for public release;

distribution is unlimited.

\title{
Energy Harvesting for
}

\section{Structural Health Monitoring Sensor Networks}

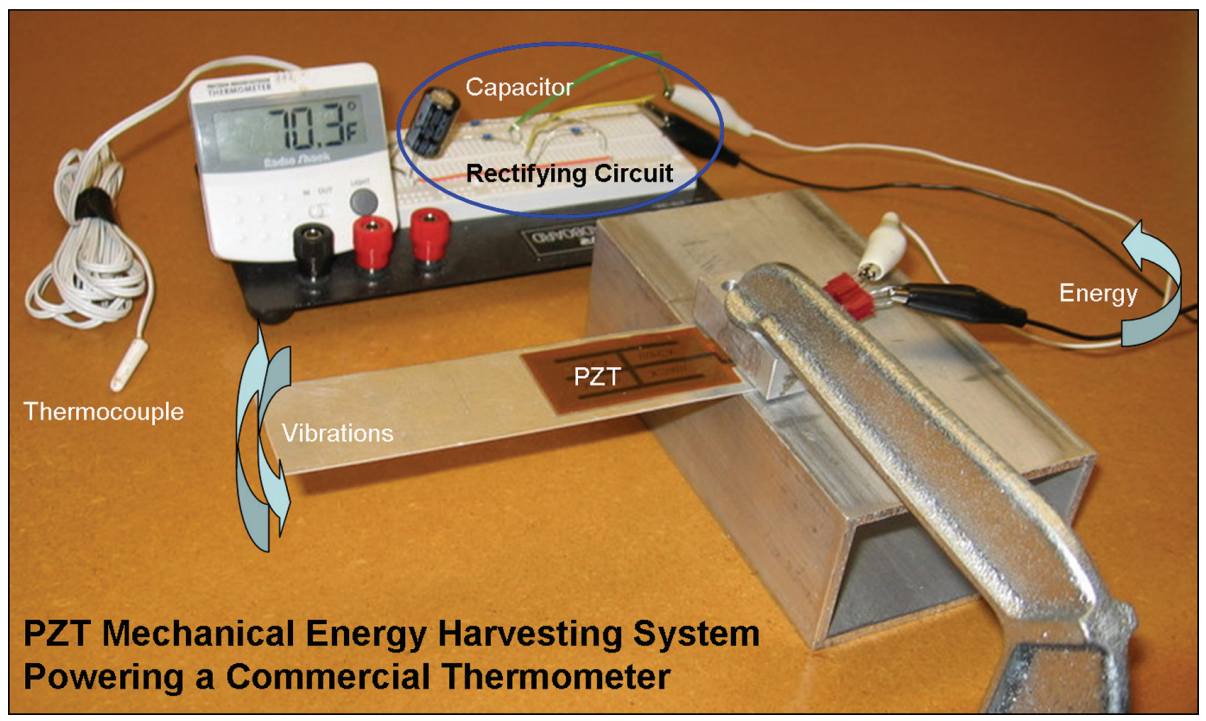


About the cover-Energy Harvesting Example: A piezoelectric patch converts ambient mechanical vibration into useful electrical energy. The electrical charge from the piezoelectric material is first rectified and then stored in a capacitor until sufficient energy is accumulated to power a commercially available thermocouple. The capacitor becomes sufficiently charged in less than $10 \mathrm{~s}$ of low-ampitude vibration.

Los Alamos National Laboratory, an Affirmative Action/ Equal Opportunity Employer, is operated by Los Alamos National Security, LLC, for the National Nuclear Security Administration of the U.S. Department of Energy under contract DE-AC52-06NA25396.

\section{MUSS \\ National Nuclear Security Administration}

This report was prepared as an account of work sponsored by an agency of the U.S. Government. Neither Los Alamos National Security, LLC, the U.S. Government nor any agency thereof, nor any of their employees make any warranty, express or implied, or assume any legal liability or responsibility for the accuracy, completeness, or usefulness of any information, apparatus, product, or process disclosed, or represent that its use would not infringe privately owned rights. Reference herein to any specific commercial product, process, or service by trade name, trademark, manufacturer, or otherwise does not necessarily constitute or imply its endorsement, recommendation, or favoring by Los Alamos National Security, LLC, the U.S. Government, or any agency thereof. The views and opinions of authors expressed herein do not necessarily state or reflect those of Los Alamos National Security, LLC, the U.S. Government, or any agency thereof. Los Alamos National Laboratory strongly supports academic freedom and a researcher's right to publish; as an institution, however, the Laboratory does not endorse the viewpoint of a publication or guarantee its technical correctness. 
LA-14314-MS

Issued: February 2007

\title{
Energy Harvesting for
}

\section{Structural Health Monitoring Sensor Networks}

\author{
G. Park \\ C. R. Farrar \\ M. D. Todd* \\ W. Hodgkiss** \\ T. Rosing $\dagger$
}

* Department of Structural Engineering, University of California, San Diego, La Jolla, CA 92093-0085.

** Department of Electrical and Computer Engineering, University of California, San Diego, La Jolla CA 92093-0701.

$\dagger$ Department of Computer Science and Engineering, University of California, San Diego, La Jolla, CA 92093-0114.

\section{Los Alamos}





\section{CONTENTS}

LIST OF FIGURES .............................................................................................. vii

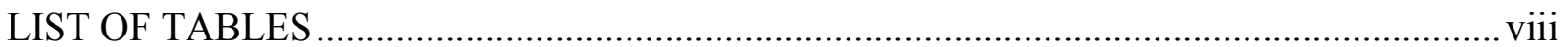

ENERGY HARVESTING FOR STRUCTURAL HEALTH MONITORING SENSOR

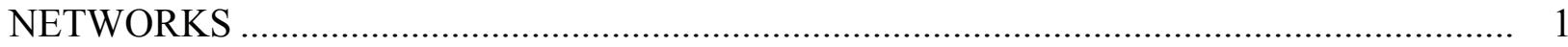

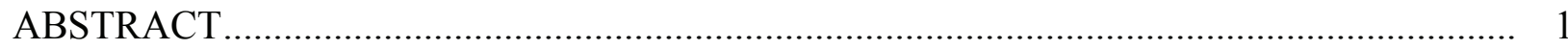

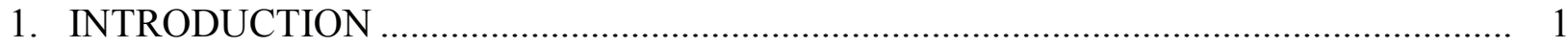

1.1. The Structural Health Monitoring Process ........................................................ 2

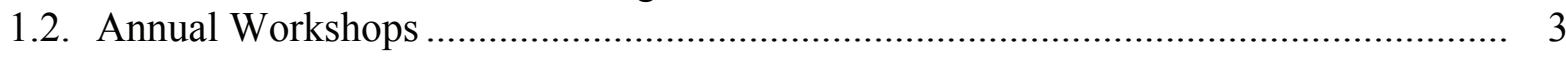

2. SENSING SYSTEM DESIGN CONSIDERATIONS FOR SHM ................................. 4

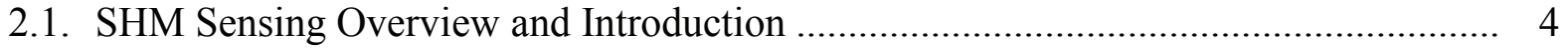

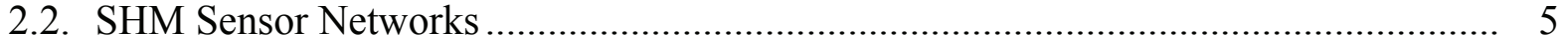

2.3. Sensor Modalities in Current SHM System Use ............................................. 6

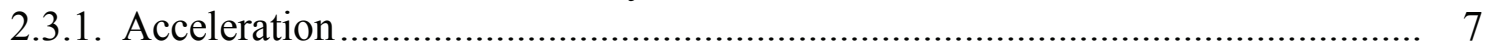

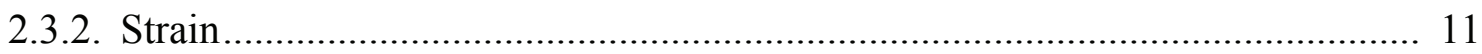

2.3.3. Fiber-Optic Strain Sensing ................................................................... 13

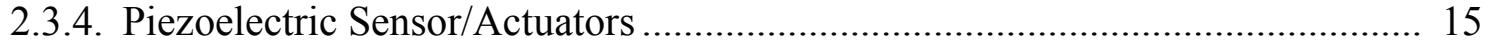

3. CURRENT SHM SENSOR NETWORK PARADIGMS ............................................. 17

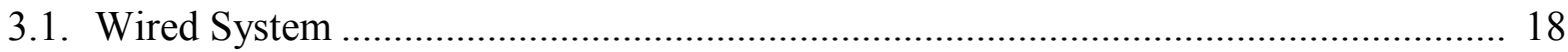

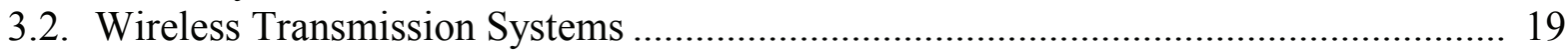

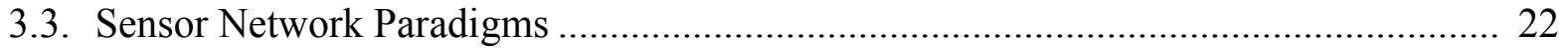

3.3.1. Sensor Arrays Directly Connected to Central Processing Hardware ................ 22

3.3.2. Decentralized Sensing and Processing with Hopping Connection..................... 22

3.3.3. Decentralized Active Sensing and Processing with Hybrid Connection........... 23

3.4. Future Sensing Network Paradigms ..................................................................... 25

3.5. Practical Implementation Issues for SHM Sensing Networks ................................. 26

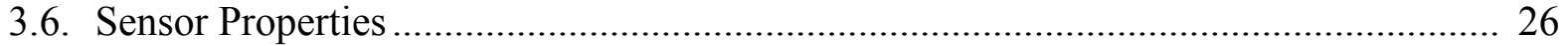

3.7. Sensor Calibration and Ruggedness ................................................................ 26

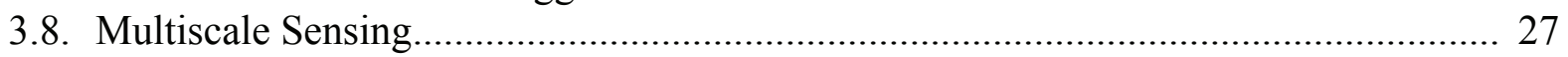

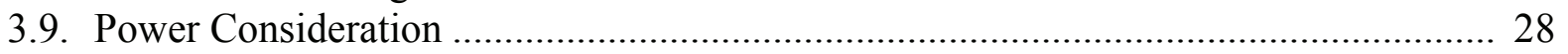


4. ENERGY DEMANDS ASSOCIATED WITH SHM SENSING SYSTEMS.................... 28

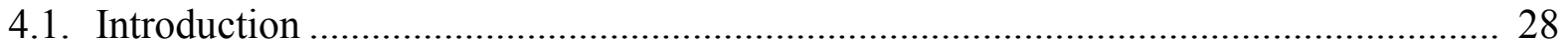

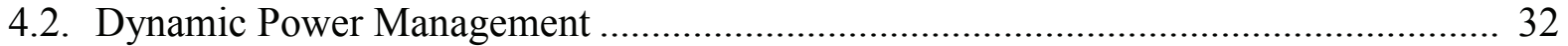

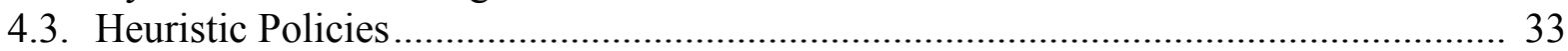

4.3.1. Timeout Policies .................................................................................... 33

4.3.2. Predictive Policies .................................................................................. 34

4.4. Stochastic Policies .................................................................................................. 34

4.5. Operating System and Cross-Layer Dynamic Power Management ........................... 36

4.6. Dynamic Voltage Scaling ..................................................................................... 37

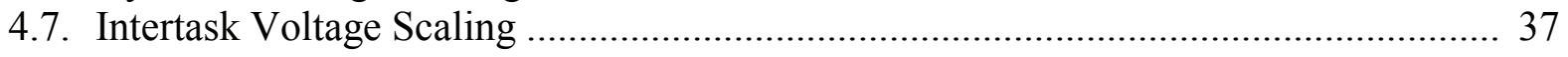

4.8. Intra-Task Voltage Scaling ........................................................................... 38

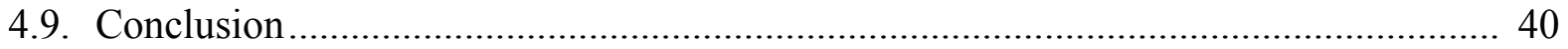

5. ENERGY HARVESTING METHODS AND APPLICATIONS FOR SHM ................... 40

5.1. Converting Mechanical Vibration to Electrical Energy ............................................ 41

5.2. Converting Thermal Energy to Electrical Energy ................................................. 49

5.3. RF Energy Harvesting .................................................................................. 51

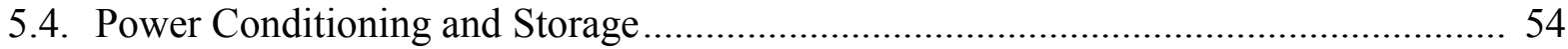

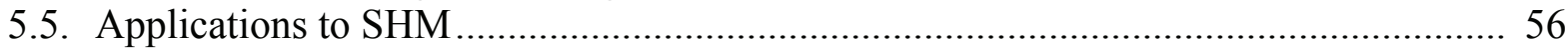

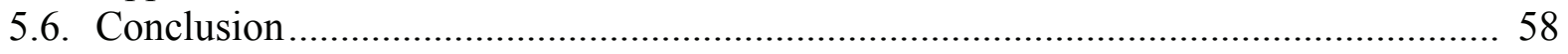

6. FUTURE RESEARCH NEEDS AND CHALLENGES ............................................... 58

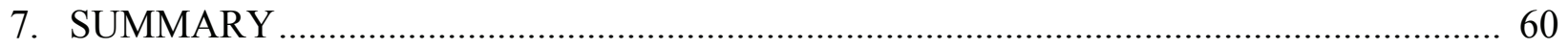

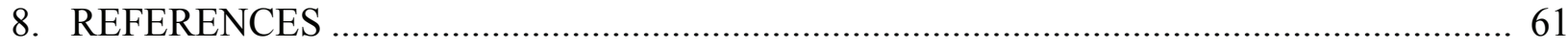

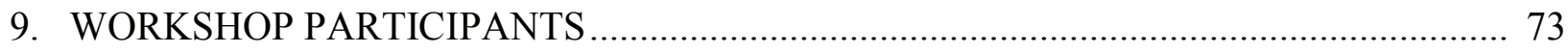

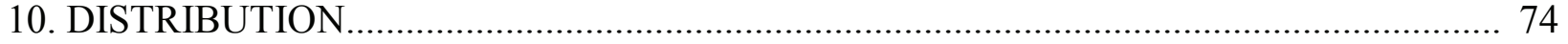




\section{List of Figures}

Figure 2.1. General sensor network architecture.............................................................. 6

Figure 2.2. Conventional piezoelectric accelerometer design.................................................. 7

Figure 2.3. Accelerometer transfer function between structure and user................................ 8

Figure 2.4. Conventional piezoelectric accelerometer signal processing. ............................... 9

Figure 2.5. 'Typical' single-axis MEMS accelerometer design (top) with two realizations integrated with CMOS circuitry (bottom, left and right)...................................... 10

Figure 2.6. Primary signal processing methods for foil strain gages. .................................... 12

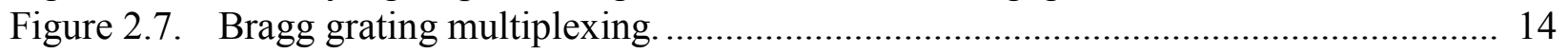

Figure 2.8. Fiber Bragg grating architecture using a tunable Fabry-Perot filter...................... 15

Figure 2.9. Some typical piezoelectric sensor/actuators from www.piezo.com (left), www.msiusa.com (center), and NASA Langley (right)......................................... 16

Figure 3.1. Conventional wired data acquisition system..................................................... 18

Figure 3.2. An active sensing system designed specifically for SHM.................................. 19

Figure 3.3. Mote sensing board mounted in programming bay. ............................................ 20

Figure 3.4. The wireless communication board displayed on the prototype SHM system....... 21

Figure 3.5. Decentralized wireless SHM system employing hopping

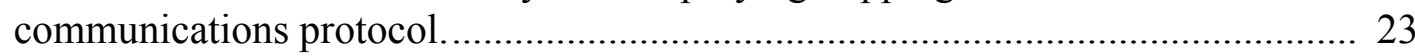

Figure 3.6. Decentralized wireless, active SHM system with energy harvesting and hybrid connection........................................................................................ 24

Figure 3.7. Relay-based hardware with optional manual controls included. ........................... 24

Figure 3.8. Active sensing network that includes energy harvesting and that is interrogated by an unmanned robotic vehicle...................................................... 25

Figure 4.1. Battery capacity vs processor performance. ........................................................ 28

Figure 4.2. Power consumption of two different embedded system designs........................... 29

Figure 4.3. Dynamic voltage scaling for a single processor. .............................................. 30

Figure 4.4. Dynamic power management for a single device............................................... 31

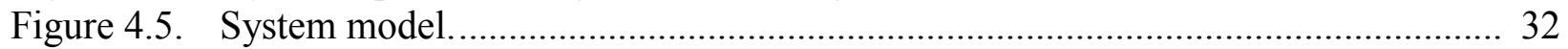

Figure 4.6. WLAN idle-state arrival tail distribution.......................................................... 35

Figure 5.1. Schematic and results of energy harvesting shoe. .................................................. 43

Figure 5.2. Energy harvesting "eel" concept............................................................................ 44

Figure 5.3. The fabricated microscale piezoelectric generator. ………................................. 45

Figure 5.4. A piezoelectric generator with a proof mass........................................................ 46

Figure 5.5. An electromagnetic generator......................................................................... 47

Figure 5.6. A prototype of sensor node with piezoelectric energy harvester........................... 48

Figure 5.7. Schematic of the Seebeck effect. ......................................................................... 49

Figure 5.8. A schematic of RF energy delivery system for a down-well pipe......................... 52

Figure 5.9. RF energy delivery test setup......................................................................... 54

Figure 5.10. Schematic of energy harvesting circuit................................................................. 54

Figure 5.11. Implementation of self-powered sensors for damage detection. ............................ 56

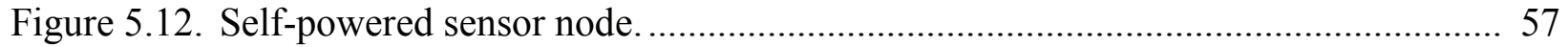

Figure 5.13. Diagram of self-powered sensor system......................................................... 58 


\section{List of Tables}

Table 5.1. Comparison of energy sources. (Source: Roundy [86])................................. 42

Table 5.2. Energy harvesting demonstrated capabilities................................................ 42

Table 5.3. Comparison elements between electromagnetic and piezoelectric systems.......... 48 


\title{
Energy Harvesting for Structural Health Monitoring Sensor Networks
}

\author{
Gyuhae Park, ${ }^{1}$ Charles R. Farrar, ${ }^{1}$ Michael D. Todd, ${ }^{2}$ William Hodgkiss, ${ }^{3}$ Tajana Rosing ${ }^{4}$
}

\begin{abstract}
This report has been developed based on information exchanges at a 2.5-day workshop on energy harvesting for embedded structural health monitoring (SHM) sensing systems that was held June 28-30, 2005, at Los Alamos National Laboratory. The workshop was hosted by the LANL/UCSD Engineering Institute (EI). This Institute is an education- and research-focused collaboration between Los Alamos National Laboratory (LANL) and the University of California, San Diego (UCSD), Jacobs School of Engineering. A Statistical Pattern Recognition paradigm for SHM is first presented and the concept of energy harvesting for embedded sensing systems is addressed with respect to the data acquisition portion of this paradigm. Next, various existing and emerging sensing modalities used for SHM and their respective power requirements are summarized, followed by a discussion of SHM sensor network paradigms, power requirements for these networks and power optimization strategies. Various approaches to energy harvesting and energy storage are discussed and limitations associated with the current technology are addressed. This discussion also addresses current energy harvesting applications and system integration issues. The report concludes by defining some future research directions and possible technology demonstrations that are aimed at transitioning the concept of energy harvesting for embedded SHM sensing systems from laboratory research to fielddeployed engineering prototypes.
\end{abstract}

\section{INTRODUCTION}

This report has been developed based on information exchanges at a 2.5-day workshop on energy harvesting for embedded structural health monitoring (SHM) sensing systems that was held June 28-30, 2005, at Los Alamos National Laboratory. The workshop was hosted by the LANL/UCSD Engineering Institute (EI). This Institute is an education- and research-focused collaboration between Los Alamos National Laboratory (LANL) and the University of California, San Diego (UCSD), Jacobs School of Engineering. The technical focus of this Institute is damage

\footnotetext{
${ }^{1}$ The Engineering Institute, Los Alamos National Laboratory, Los Alamos, New Mexico 87545.

${ }^{2}$ Department of Structural Engineering, University of California, San Diego, La Jolla, CA 92093-0085.

${ }^{3}$ Department of Electrical and Computer Engineering, University of California, San Diego, La Jolla, CA 92093-0701.

${ }^{4}$ Department of Computer Science and Engineering, University of California, San Diego, La Jolla, CA 92093-0114.
} 
prognosis (DP), a multidisciplinary engineering science concerned with assessing the current condition and predicting the remaining life of aerospace, civil, and mechanical infrastructure. Structural Health Monitoring, which is the process of identifying damage in structures and tracking its evolution, is one of the technologies necessary to perform DP.

\subsection{The Structural Health Monitoring Process}

Structural health monitoring (SHM) is the process of detecting damage in structures. The goal of SHM is to improve the safety and reliability of aerospace, civil, and mechanical infrastructure by detecting damage before it reaches a critical state. To achieve this goal, technology is being developed to replace qualitative visual inspection and time-based maintenance procedures with more quantifiable and automated damage assessment processes. These processes are implemented using both hardware and software with the intent of achieving more cost-effective condition-based maintenance. A more detailed general discussion of SHM can be found in [1].

The authors believe that all approaches to SHM, as well as all traditional nondestructive evaluation procedures (e.g., ultrasonic inspection, acoustic emissions, active thermography) can be cast in the context of a statistical pattern recognition problem [2]. Solutions to this problem require four steps: (1) Operational evaluation, (2) Data acquisition, (3) Feature extraction, and (4) Statistical modeling for feature classification.

A necessary first step to developing an SHM capability is Operational Evaluation. This part of the SHM solution process attempts to answers four questions regarding the implementation of an SHM system: (1) What are the life safety and/or economic justifications for monitoring the structure? (2) How is damage defined for the system being monitored? (3) What are the operational and environmental conditions under which the system of interest functions? (4) What are the limitations on acquiring data in the operational environment? Operational evaluation defines, and to the greatest extent possible quantifies, the damage that is to be detected. It also defines the benefits to be gained from deployment of the SHM system. This process also begins to set limitations on what will be monitored and how to perform the monitoring as well as tailoring the monitoring to unique aspects of the system and unique features of the damage that is to be detected.

The Data Acquisition portion of the SHM process involves selecting the excitation methods; the sensor types, numbers, and locations; and the data acquisition/storage/processing/transmittal hardware. The actual implementation of this portion of the SHM process will be application specific. A fundamental premise regarding data acquisition and sensing is that these systems do not measure damage. Rather, they measure the response of a system to its operational and environmental loading or the response to inputs from actuators embedded with the sensing system. Depending on the sensing technology deployed and the type of damage to be identified, the sensor readings may be more or less directly correlated to the presence and location of damage. Data interrogation procedures (feature extraction and statistical modeling for feature classification) are the necessary components of an SHM system that convert the sensor data into information about the structural condition. Furthermore, to achieve successful SHM, the data acquisition system will have to be developed in conjunction with these data interrogation procedures. 
A damage-sensitive feature is some quantity extracted from the measured system response data that is correlated with the presence of damage in a structure. Ideally, a damage-sensitive feature will change in some consistent manner with increasing damage level. Identifying features that can accurately distinguish a damaged structure from an undamaged one is the focus of most SHM technical literature [3, 4]. Fundamentally, the Feature Extraction process is based on fitting some model, either physics-based or data-based, to the measured system response data. The parameters of these models or the predictive errors associated with these models then become the damage-sensitive features. An alternate approach is to identify features that directly compare the sensor waveforms or spectra of these waveforms measured before and after damage. Many of the features identified for impedance-based and wave propagation-based SHM studies fall into this category $[5,6,7,8]$.

The portion of the structural health monitoring process that has received the least attention in the technical literature is the development of statistical models to enhance the damagedetection process. Statistical modeling for feature classification is concerned with the implementation of the algorithms that analyze the distributions of the extracted features in an effort to determine the damage state of the structure. The algorithms used in statistical model development usually fall into three general categories: (1) Group Classification, (2) Regression Analysis, and (3) Outlier Detection. The appropriate algorithm to use will depend on the ability to perform supervised or unsupervised learning. Here, supervised learning refers to the case where examples of data from damaged and undamaged structures are available. Unsupervised learning refers to the case where data are only available from the undamaged structure. The statistical models are typically used to answer a series of questions regarding the presence, location, type, and extent of damage.

Inherent in the data acquisition, feature extraction, and statistical modeling portions of the SHM process are data normalization, cleansing, fusion, and compression. As it applies to SHM, data normalization is the process of separating changes in sensor reading caused by damage from those caused by varying operational and environmental conditions [9]. Data cleansing is the process of selectively choosing data to pass on to, or reject from, the feature selection process. Data fusion is the process of combining information from multiple sensors in an effort to enhance the fidelity of the damage-detection process. Data compression is the process of reducing the dimensionality of the data, or the feature extracted from the data, in an effort to facilitate efficient storage of information and to enhance the statistical quantification of these parameters. These four activities can be implemented in either hardware or software and usually a combination of the two approaches is used.

\subsection{Annual Workshops}

A new component to the EI is an annual workshop that focuses on various aspects of the broad area of DP. Among the important topic areas identified for discussions are sensing and data acquisition, data interrogation procedures, and predictive modeling as these topics relate to DP. The 2005 workshop focus was on energy harvesting as it relates to embedded networked sensing for SHM. As the sensor network hardware evolves, the possibility of embedding these networks in all types of aerospace, civil, and mechanical infrastructure is becoming both technically and economically feasible. Such networks can provide the dense array of sensors necessary to detect incipient damage, which in turn will allow maintenance to be performed 
before the system performance and/or safety are compromised. Ideally, such embedded sensing systems will contain sensors, actuators (for active sensing), a processing capability, memory, telemetry and a sensing system diagnostic capability. However, the concept of "embedded" sensing cannot be fully realized if the systems will require access to AC power or if batteries have to be periodically replaced. Therefore, there is a need to harvest and store ambient sources of energy (mechanical or thermal) in an effort to make these embedded systems as autonomous as possible. Although energy harvesting for large-scale alternative energy generation using wind turbines and solar cells is mature technology, the development of energy harvesting technology on a scale appropriate for small embedded sensing systems is still in the developmental stages, particularly when applied to SHM sensing systems. This report will summarize the state-of-theart in energy harvesting as it has been applied to SHM embedded sensing systems.

\section{SENSING SYSTEM DESIGN CONSIDERATIONS FOR SHM}

\subsection{SHM Sensing Overview and Introduction}

Within the SHM paradigm presented previously, the first line of attack is clearly the establishment of an appropriate sensor network that can adequately observe the system dynamics for suitable signal processing and feature extraction. Sensor networks, generally speaking, contain three main components: the sensing unit itself, communications, and computation (hardware and, as appropriate, software control and processing algorithms) [10].

The goal of any SHM sensor network system is to make the sensor reading as directly correlated with, and as sensitive to, damage as possible. At the same time, one also strives to make the sensors as independent as possible from all other sources of environmental and operational variability, and, in fact, independent from each other (in an information sense) to provide maximal data from minimal sensor array outlay. To best meet these requirements, the following design parameters must be defined, as much as possible, a priori:

- Types of data to be acquired,

- Sensor types, numbers, and locations,

- Bandwidth, sensitivity, and dynamic range,

- Data acquisition/telemetry/storage system,

- Power requirements,

- Sampling intervals (continuous monitoring versus monitoring only after extreme events or at periodic intervals),

- Processor/memory requirements, and

- Excitation source needs (for active sensing). 
Fundamentally, there are five issues that control the selection of hardware to address these sensor system design parameters:

- The length scales on which damage is to be detected,

- The time scale on which damage evolves,

- Effects of varying and/or adverse operational and environmental conditions on the sensing system,

- Power availability, and

- Cost.

In addition, the feature extraction, data normalization and statistical modeling portions of the process can greatly influence the definition of the sensing system properties. Before such decisions can be made two important questions must be addressed. First, one must answer the question, "What is the damage to be detected?" The answer to this question must be provided in as quantifiable a manner as possible and address issues such as (i) type of damage (e.g., crack, loose connection, corrosion); (ii) threshold damage size that must be detected; (iii) probable damage locations; and (iv) anticipated damage growth rates. The more specific and quantifiable this definition, the more likely it is that the sensor budget can be optimized to produce a system that has the greatest possible fidelity for damage detection. Second, an answer must be provided to the question, "What are the environmental and operational sources of variability that must be taken into account?" To answer this question, one will not only have to have some ideas about the physical sources per se, but one will also have to have thought about how to accomplish data normalization. Typically, data normalization will be accomplished through some combination of sensing system hardware and data interrogation software. However, these hardware and software approaches will not be optimal if they are not done in a coupled manner.

\subsection{SHM Sensor Networks}

From the discussion in Section 2.1, it is clear that the ability to convert sensor data into structural health information is directly related to the coupling of the sensor system hardware with the data interrogation procedures in an integrated network. A very generalized sensor network paradigm is shown in Figure 2.1. The most common general approach in this function is a conventional wired sensor network, where some number of a given transducer type (e.g., accelerometers) are connected via conductive cabling to a centralized data processing and multiplexing unit. Each sensor is effectively independent of other sensors in the network - each sensor has its own cabling - and controlled synchronized interrogation of the entire network is achieved only through the central unit. This interrogation is typically only passive as well, meaning that the sensors individually provide information to the central unit, but the central unit cannot pass information back to an individual sensor. In applications demanding control or feedback, actuator arrays (e.g., piezoelectric actuators) can take the place of some of the passive sensor arrays, though typically each node (sensor or actuator) is still individually wired and connected to a central unit. 


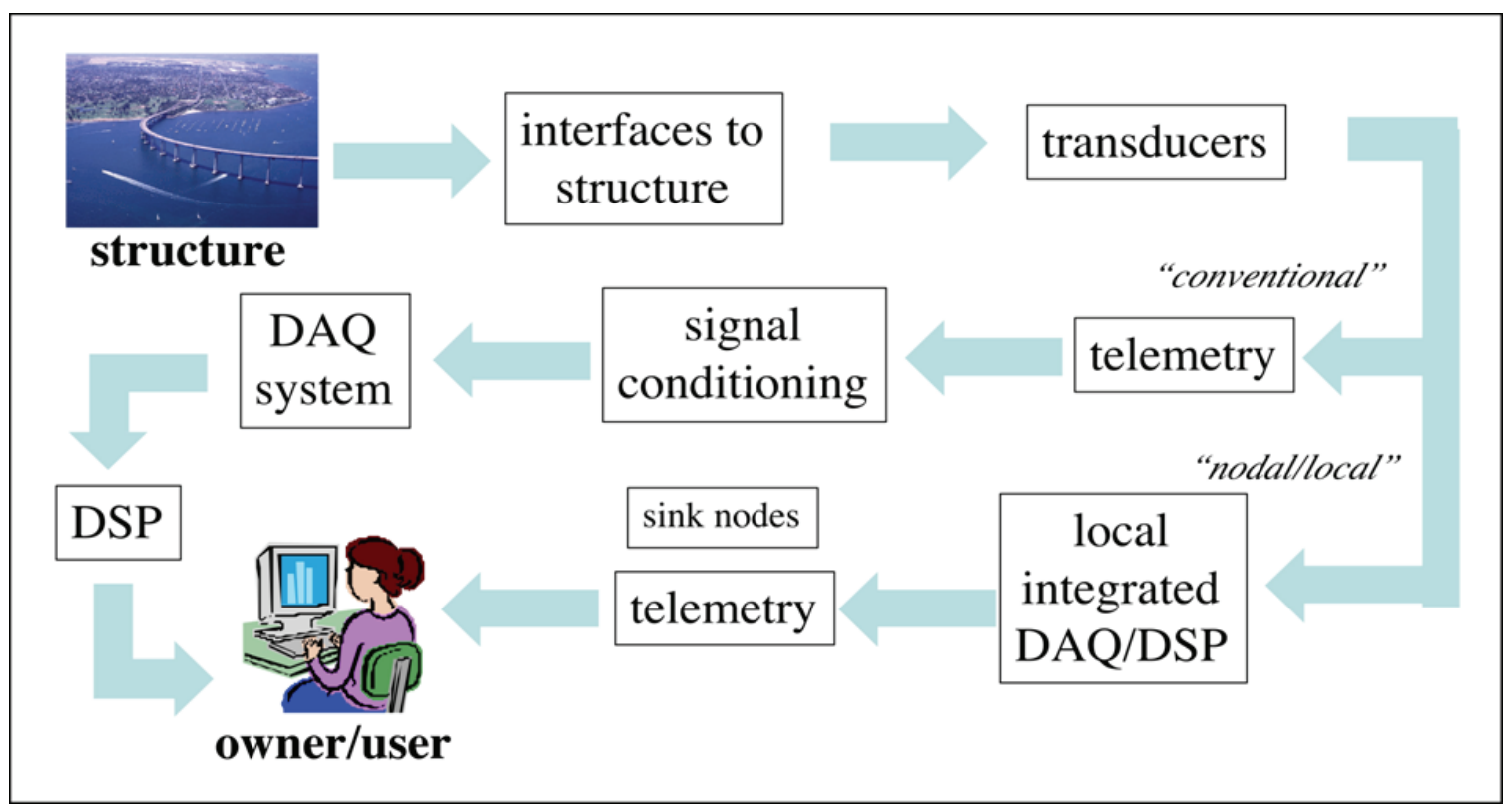

Figure 2.1. General sensor network architecture.

While the majority of sensor networks in use today employ a wired architecture, development and deployment of wireless sensor networks has exploded in recent years. Wireless communication protocols are now standardized with such protocols as IEEE 802.11 through 802.15, and bandwidths are now approaching that of conventional wired networks. Furthermore, increases in chip real estate and processor production capability have reduced the power requirements for both computing and communication. In fact, sensing, communication, and computing can now be performed on a single chip, reducing the cost further and permitting economically viable high-density sensor networks. All of these advances have yielded wireless sensor networks that increasingly meet the original visions for these networks: (1) a large number of individual sensor nodes, densely deployed in possibly random configurations in the sensing environment; (2) the capability for self-organization and near-neighbor awareness so that information exchange between an individual node and a user may be achieved via point-to-point hopping protocols; (3) cooperation between sensor nodes, where they use local processing capability to perform data fusion or other computational duties and then transmit only required or partially processed data onward.

\subsection{Sensor Modalities in Current SHM System Use}

The sensing component (transducer) refers to the actual transduction mechanism that converts a physical field (such as acceleration) into a measurable form (usually an electrical potential difference). If the sensing system involves actuation, then the opposite is required, i.e., a voltage command is converted into a physical field (usually displacement). The most common measurements currently made for SHM purposes by far are, in order of use:

1. Acceleration (with piezoelectric, piezoresistive, capacitive or fiber-optic accelerometers).

2. Strain (resistive foil, fiber-optic, or piezoelectric patch gages).

3. Lamb wave/impedance (piezoelectric patches). 


\subsubsection{Acceleration}

Making local acceleration measurements using some form of accelerometer is by far the most common approach today. This situation is primarily the result of the relative maturity and commercial availability of accelerometer hardware, such as a piezoelectric accelerometer. A cutaway view of a piezoelectric accelerometer is shown in Figure 2.2. These devices are designed such that an external displacement input to the housing inertially induces shape changes in a piezoelectric crystal.

Piezoelectric crystals are made from ferroelectric materials (such as naturally occurring quartz or man-made lead zirconate titanate, PZT) that induce electric charge (electric dipoles at the molecular level) when mechanically strained. The inertially induced strain on the crystal is contained within a damping block and an inertial seismic mass for frequency response tuning, and the resulting charge is picked up by wiring. Several components, as shown on the left of Figure 2.3, actually comprise the overall transfer function between host structure and data acquisition system (the network), including structure/transducer coupling (usually epoxy, wax, or direct bolted connection), the housing design itself, the seismic mass, the crystal, the contact wires, and the cabling. All of these have inherent sources of variability, as shown in italics in Figure 2.3.

These accelerometers are designed to be used within a conventional wired network, and each individual sensor output voltage must be transferred to a centralized data acquisition unit containing appropriate charge amplification, analog-to-digital converters, signal processing (e.g., antialiasing or filtering), and demultiplexing. There are two general designs for the piezoelectric sensor: high and low impedance [11]. High-impedance designs require a charge amplifier or external impedance converter for charge-to-voltage conversion. The charge amplifier consists of a high-gain inverting voltage amplifier with a field-effect transistor at its input for high insulation resistance. Low-impedance designs use the same piezoelectric sensing element as high-impedance units, but they also incorporate a miniaturized built-in charge-tovoltage converter. They also require an external power supply coupler, which provides the constant current excitation required for linear operation over a wide voltage range and also decouples the bias voltage from the output. Both the power into and the signal out of the sensor are transmitted over this cable.

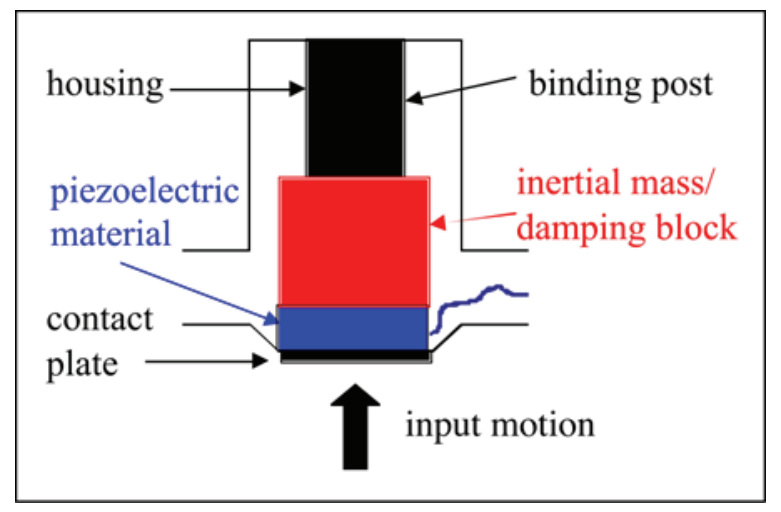

Figure 2.2. Conventional piezoelectric accelerometer design. 


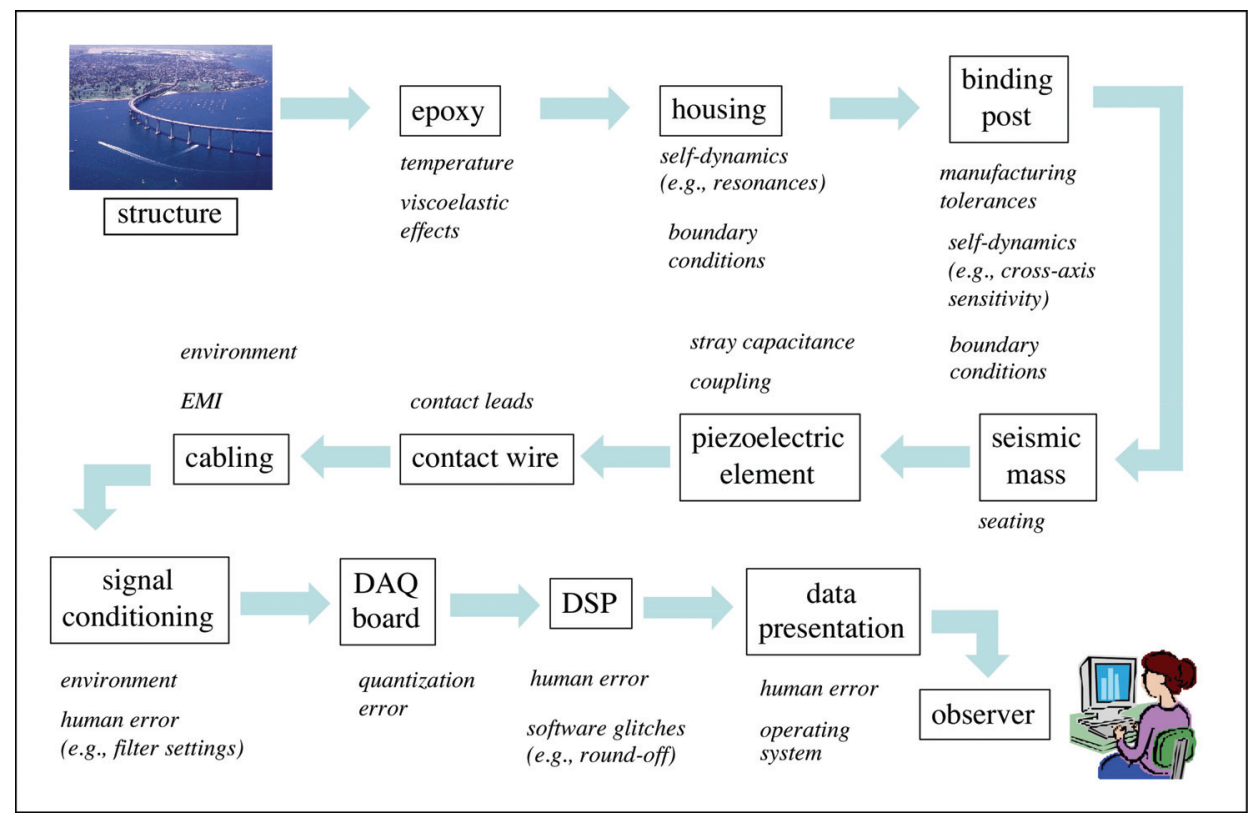

Figure 2.3. Accelerometer transfer function between structure and user.

Figure 2.4 shows these designs. In high-impedance systems, time constant, gain, and signal normalization are all controlled with an external charge amplifier. High-impedance systems also usually have longer time constants that allow easy short-term static calibration, and they have a wider operating temperature range resulting from no built-in electronics. In some designs, an alternative method for charge processing utilizes an external impedance converter, which exploits the high temperature range of the sensor and offers the convenience and costeffectiveness of a coupler (as compared to a charge amplifier).

In general, low-impedance systems are less versatile than high-impedance systems because the sensors have an internally fixed range and time constant. However, for applications where bandwidth and operating temperature range are well-defined and constant, low-impedance systems may cost less and can be used with general-purpose cables in environments where high humidity and/or contamination degrade the high insulation resistance required for highimpedance sensors. Also, the ability to tolerate longer cable lengths between sensor and signal conditioner and compatibility with a wide range of signal display devices are further advantages of low-impedance sensors (as far more such devices are already impedance-matched with such sensors).

These piezoelectric measuring systems are active electrical systems, meaning that the crystals produce an electrical output only when they experience a change in load; this means they cannot detect true static response. Essentially the sensor behaves as a single-degree-of-freedom under-damped oscillator. This behavior means that the sensor is subject to resonance phenomena (frequency-dependent sensitivity in the resonance band, phase distortion, etc.) and operates well as an accelerometer in a finite frequency band below the resonant region, but not all the way to DC. As mentioned, these systems also have characteristic time constants, which are defined to be the discharge time of the corresponding AC circuit. Time constants are usually partially selectable by resistors in the control circuit, although this depends on the design (low-impedance vs high-impedance). These sensors can be designed to measure responses up to many $\mathrm{kHz}$ with micro-g sensitivity. 


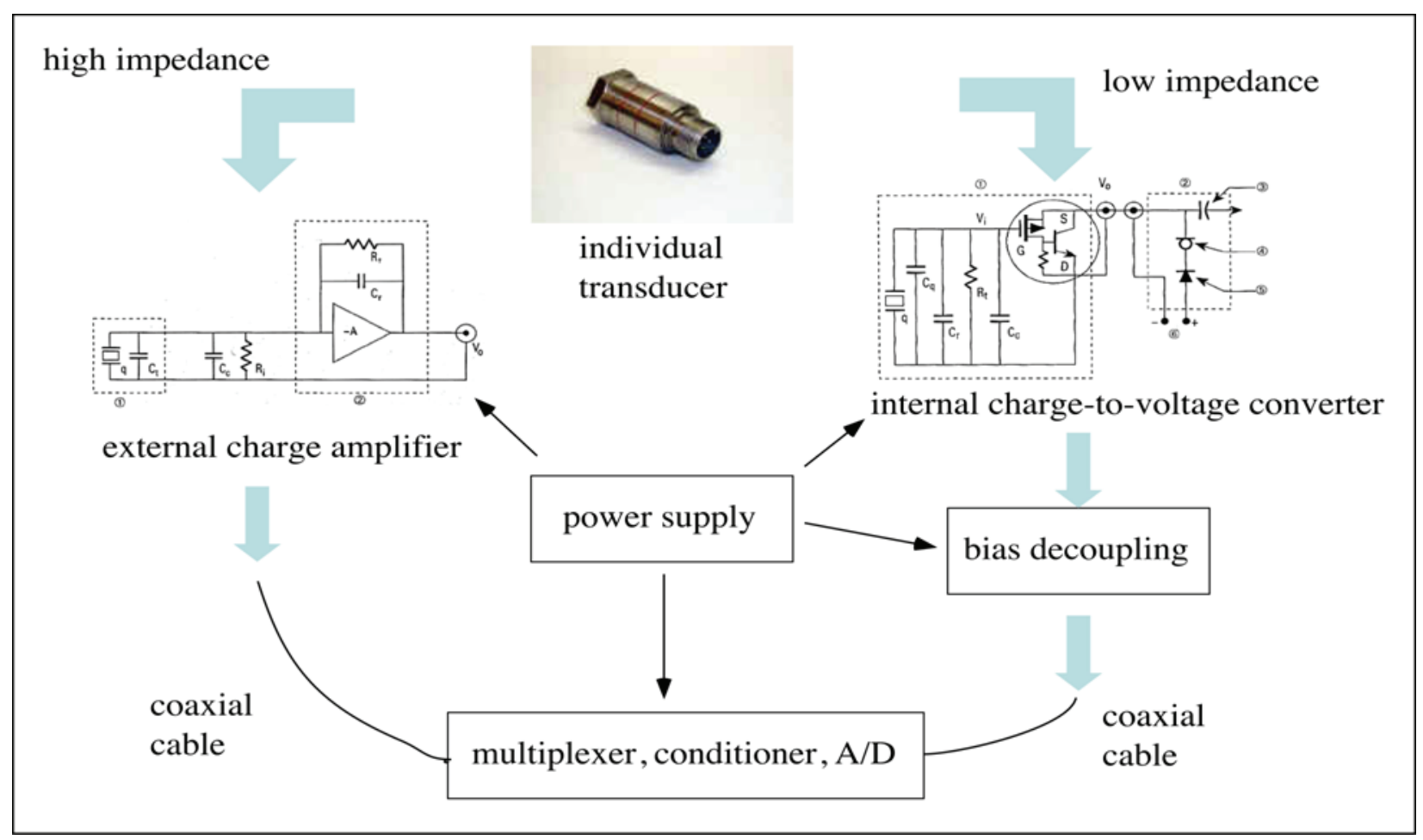

Figure 2.4. Conventional piezoelectric accelerometer signal processing.

Although there are numerous materials that exhibit the piezoelectric effect, quartz is typically preferred in sensor designs because of the following properties:

- Elastic stress limit of about $140 \mathrm{MPa}(20 \mathrm{ksi})$;

- Temperature resistance greater than $900^{\circ} \mathrm{F}$;

- Extreme rigidity, high linearity, minimal hysteresis, and constant sensitivity over a wide frequency and temperature range; and

- Ultrahigh insulation resistance (on the order of $1 \mathrm{k} \Omega$ ), allowing reliable low-frequency measurements $(<1 \mathrm{~Hz})$.

Although the majority of measurements made with these quartz-based sensors are acceleration, the transfer functions for measuring force and pressure are very similar, and only minor modifications are required to measure these kinetic quantities. Quartz piezoelectric sensors consist essentially of thin slabs or plates cut in a precise orientation to the crystal axes, depending on the application, because the crystals respond in both transverse and shear to applied load. The shear cut is used for multicomponent force and acceleration measuring sensors. Other specialized cuts include the transverse cut for some pressure sensors and the polystable cut for high-temperature pressure sensors. The finely lapped quartz elements are assembled either singly or in stacks and preloaded in some manner. The quartz package generates a charge signal that is directly proportional to the sustained force (and thus, acceleration). 
The energy consumed by these devices themselves is very small because of their passive nature, but the centralized multiplexing, amplification, and signal conditioning units required to obtain usable raw data can often have power requirements that approach $1 \mathrm{~W}$. A typical 4-channel power supply delivers $3-30 \mathrm{~mA}$ of current at $30 \mathrm{~V}$, equating to $0.9 \mathrm{~W}$ in the largest case; power requirements go up with large channel counts so that very large $(\sim 100)$ accelerometer arrays may have power requirements measuring tens of watts.

Accelerometer designs have also taken advantage of manufacturing, fabrication, and microelectronics developments at the micrometer scale that have led to the microelectromechanical systems (MEMS) revolution. The fabrication of MEMS devices begins with a very thin film growth (usually epitaxial silicon, a silicon oxide or nitride, a polysilicon complex, or even a metal) upon a silicon substrate. Specific dopants are usually introduced to control layer properties. Lithography (for deposition) and etching (for targeted removal) techniques are then used to implant desired mechanical patterns into the layer using photomasks. Significantly advanced surface micromachining processes have even produced three-dimensional structure fabrication [12]. These techniques, along with continuing complementary-symmetry metal-oxide semiconductor (CMOS) development at $60 \mathrm{~nm}$ and $45 \mathrm{~nm}$, have allowed for the development of numerous MEMS system-on-a-chip (SOC) sensor solutions.

MEMS accelerometers have clearly been the most widely developed MEMS SOC modality, with millions used per year by the automotive industry alone for airbag deployment systems. MEMS accelerometers consist of the same basic components as any accelerometer, namely a proof (inertial) mass suspended somehow by elastic elements (springs). The top of Figure 2.5 shows this design conceptually, with two realizations integrated with CMOS circuitry below it. Often a wafer layer (or subset thereof) itself is the proof mass, coupled by thin 'beams' (the springs) to small capacitive plates that transform deflection-induced capacitance shifts to a voltage. This capacitive design is by the far the most widely used, although other MEMS accelerometer designs include piezoresistive, ferroelectric, optical, and tunneling [13]. Multiaxis versions of these designs, particularly the capacitive one, are commercially available.
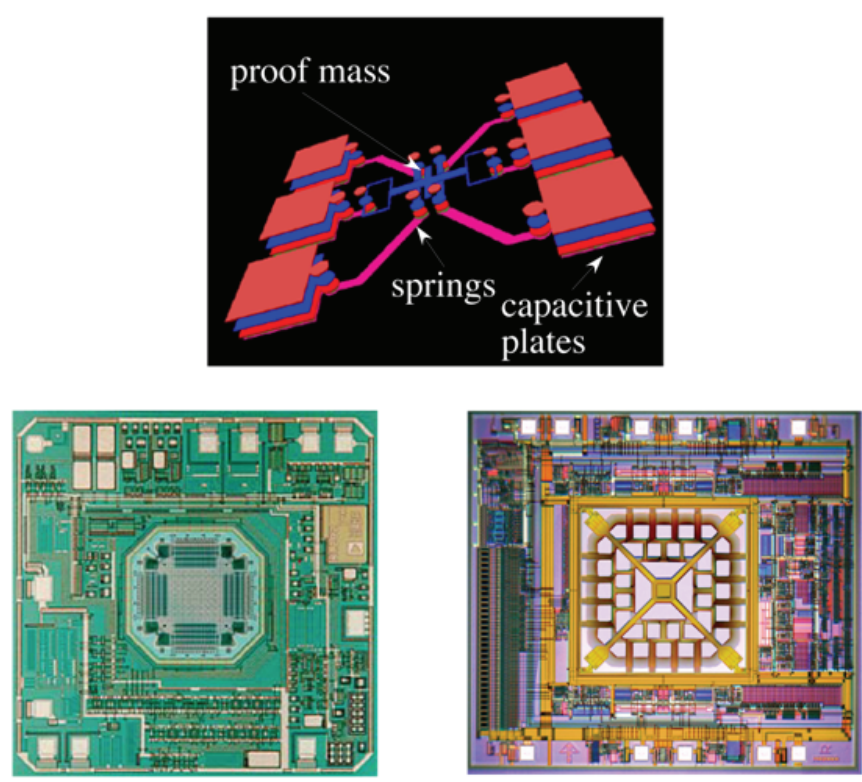

Figure 2.5. 'Typical' single-axis MEMS accelerometer design (top) with two realizations integrated with CMOS circuitry (bottom, left and right). 
Performance characteristics for MEMS accelerometers are comparable to those of conventional accelerometers, although their very small length scale makes them susceptible to Johnston noise arising from Brownian motion (usually neglected in larger sensors). Vacuum packaging of the device usually minimizes sensitivity to this noise. By tuning the resonant frequency and damping characteristics, MEMS accelerometers have performed with wide dynamic ranges (nano- $g$ to hundreds of kilo- $g$ in shock-specific designs), noise floors approaching $25 \mathrm{ng} / \mathrm{Hz}^{1 / 2}$, and bandwidths into the $\mathrm{kHz}$ range (prior to first resonance). This performance is typically achieved with power usage in the $\mathrm{mW}$ range, depending on signal conditioning. Nonetheless, MEMS accelerometer use in SHM applications is significantly less than conventional accelerometer use, primarily because of the comparative lack of commercialization (availability) and ruggedness/robustness issues for many field applications.

\subsubsection{Strain}

Second to measurements of acceleration for SHM is the measurement of strain. Strain is a nondimensional measure of an object's deformation resulting from an applied stress. More formally, strain is defined as the displacement per unit length of the object, and strain gages have a finite gage length that serves as this normalizing factor. As such, the strain gage actually gives an average strain reading over the length of the gage. Like accelerometers, strain gages are a mature technology. The most common strain gage technology is the resistive kind: Lord Kelvin in 1856 first noted that some metallic conductors' resistive properties change as a function of applied strain, and this effect was put into practical use by the 1930s. In practice, other properties such as capacitance or inductance also change with applied strain, and variations on the resistive theme have been commercialized as well, though their sensitivity to other measurands, mounting requirements, and complex circuitry have limited their application. Bulk optical methods, taking advantage of interference patterns produced by optical flats, are very accurate and highly sensitive, but the technique is delicate and cannot withstand industrial applications in many cases.

The typical foil resistive-type gage consists of a wire grid network (the resistor) embedded on an elastic layer, which in turn is bonded with an epoxy layer onto the object of interest. When the object deforms under load, the deformation is transferred to the wire network, causing resistive changes in the material. Typical materials include copper-nickel alloys, nickel-chrome alloys, platinum alloys (usually tungsten), nickel-iron alloys, or nickel-chrome alloys, foils, or semiconductor materials. The most popular alloys used for strain gages are copper-nickel alloys and nickel-chrome alloys. Piezoresistive effects in semiconductor materials such as germanium and silicon are much larger (up to 100 times more sensitive than metallic gages) and such semiconductor gages may be made much smaller than metallic ones, but the response is very nonlinear (up to $20 \%$ deviation from linearity) and the temperature sensitivity is much larger. Two further improvements in strain gage technology include the thin-film strain gage and diffused semiconductor gages. With thin-film gages, an electrical insulating material is directly deposited onto the object surface, and then the strain gage gets deposited onto the insulation layer; vacuum deposition or sputtering techniques are used to molecularly bond the layers, eliminating the need for an epoxy. The diffused semiconductor gages eliminate bonding agents altogether through photolithography masking techniques and solid-state diffusion of boron to molecularly bond the resistance elements. Electrical leads are directly attached to the grid. Despite these alternative piezoresistive designs, bonded metallic resistance strain gages have the best reputation and are the most widely used. They are relatively inexpensive, can achieve 
overall accuracy of better than $\pm 0.10 \%$, are available in a short gage length, have only moderate thermal sensitivity, and have good response. They may be operated in temperature regions from cryogenic to those found in jet engine turbines. Bonded resistance strain gages can be used to measure both static and dynamic strain, as the inherent time constants in the material are negligibly small.

The changes in the resistive characteristics of metallic foil gages to applied strain are very small, so signal processing is required to convert these changes into voltages that are detectable and able to be processed by normal data acquisition systems. One of the most popular detection circuits is the Wheatstone bridge, shown to the left in Figure 2.6. The resistor network is arranged such that the transfer function gives zero net voltage drop across the terminals when $R_{1} / R_{3}=R_{\mathrm{G}} / R_{2}$; thus, when the resistor corresponding to $R_{\mathrm{G}}$ (denoting the placement of the strain gage) senses a resistance change (ostensibly due to applied mechanical strain), the bridge becomes unbalanced, yielding a potential difference across the output terminals. This network is very sensitive to small changes in $R_{\mathrm{G}}$. Other resistors may be made gages as well, depending on the application; various combinations of resistors will go unbalanced to account for effects such as temperature fluctuations and tension/compression differences. A Chevron bridge, shown in the middle of Figure 2.6, is a multiple-channel configuration that switches between bridge-arm networks periodically to compensate for imbalances.

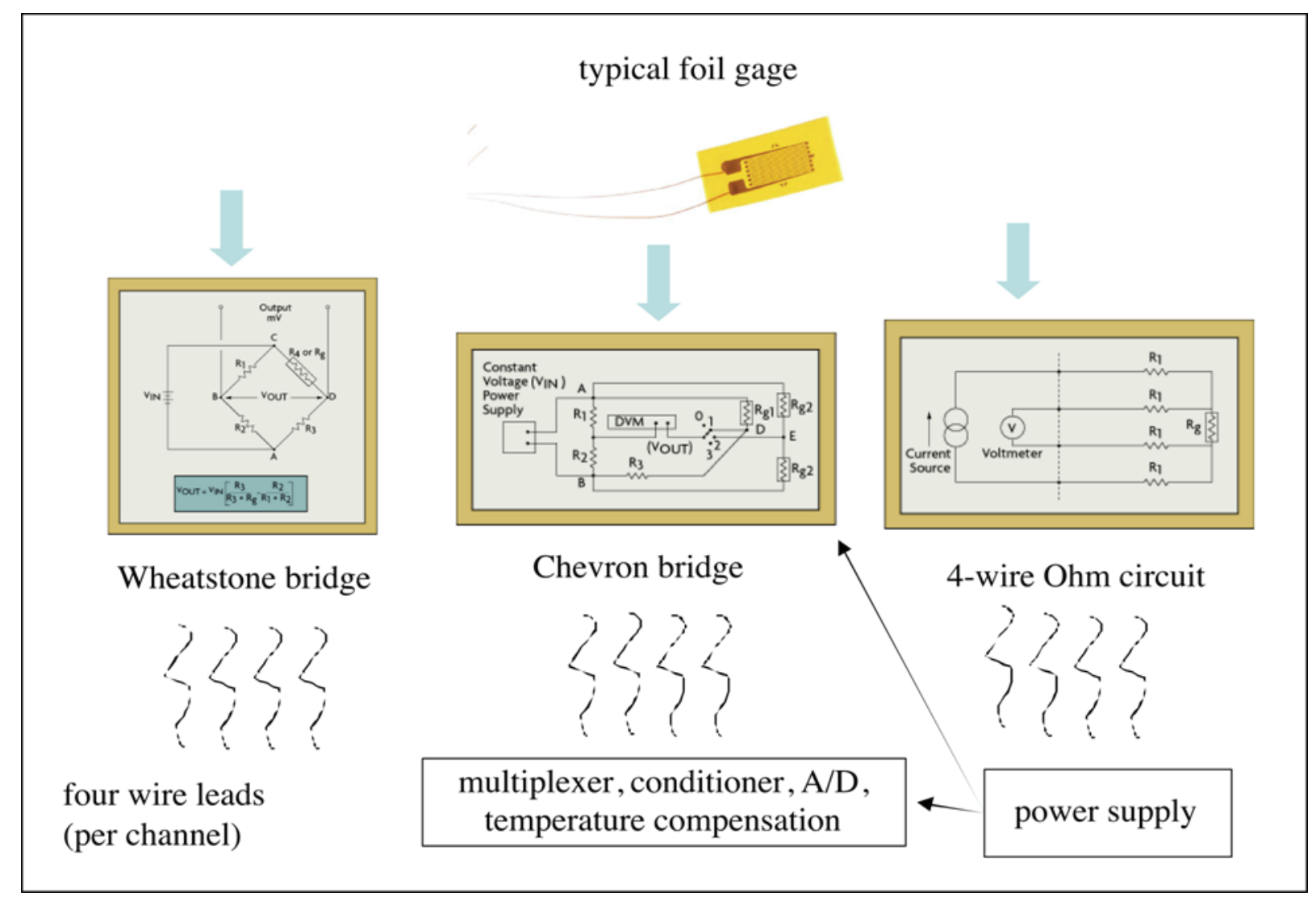

Figure 2.6. Primary signal processing methods for foil strain gages. 
A 4-wire Ohm circuit is also shown to the right in Figure 2.6. A 4-wire Ohm circuit installation might consist of a voltage measurement device (typically, the data acquisition unit), a current source, and four lead resistors in series with a gage resistance $R_{\mathrm{G}}$. A small $(\sim 1 \mathrm{~mA})$ current is supplied to the circuit, and the voltage drop across $R_{\mathrm{G}}$ is compared to the absolute resistance value computed at the data acquisition system from the values of current and voltage.

A baseline characterization is usually done by first measuring the value of gage resistance in an unstrained condition and then making a second measurement with known strain applied. The difference in the measured gage resistances divided by the unstrained resistance gives a fractional value of the strain, which may be combined with the gage factor for $R_{\mathrm{G}}$ to get strain. This design is also suitable for automatic voltage offset compensation. The voltage is first measured when there is no current flow and compared to the voltage reading when current is flowing. The resulting difference is then used to compute the gage resistance. Because of their sensitivity, four-wire strain gages are typically used to measure low frequency dynamic strains. Resolution on the order of a few microstrain is typical with these bridge circuits. Foil gage systems consume power at a level very commensurate with piezoelectric accelerometers; this is typically about $1 \mathrm{~W}$ for 3-4 channels, although the number depends on the specific input impedance of the bridge circuit being used.

\subsubsection{Fiber-Optic Strain Sensing}

Although foil resistive gages dominate current market usage, the last several years have witnessed an explosion of commercially available fiber-optic solutions to strain measurement. The fiber-optic communications revolution of the late 1990s led to great improvement in component technologies at lower costs, and the sensor development community piggy-backed on these advances. The two dominant fiber-optic technologies are direct fiber interferometry and fiber Bragg gratings (FBGs) [14]. The former method is older, but its relative complexity (despite being several orders of magnitude more sensitive than foil gages) and low multiplexing capability have limited its use to specialized military (and some industrial) applications. Most commercial systems today take advantage of FBG technology.

The FBGs are intrinsic structures that may be photowritten into the fiber. Silica glass with germanium doping is absorptive in the ultraviolet range, and such irradiation of the fiber core will cause an essentially permanent change in the refractive properties of the core. If the irradiation is performed in a spatially periodic fashion, then a series of gratings (a periodic change in refractive index) is introduced in the fiber core, and these act like a local bandstop optical filter. The physical periodicity is chosen so that when broadband light in the infrared range $(\sim 1300-1600 \mathrm{~nm})$ is propagated down the fiber core, a narrowband component (width $\sim 0.2 \mathrm{~nm}$ ) is rejected and reflected. The central wavelength of this narrowband component is directly proportional to the spacing in the FBG at that location so that as spacing physically changes (due to strain), the reflected wavelength will shift in proportion. In this way, tremendous multiplexing may be achieved: several FBGs may be individually photowritten into a single optical fiber with each one written to reflect at a unique wavelength (Figure 2.7). 


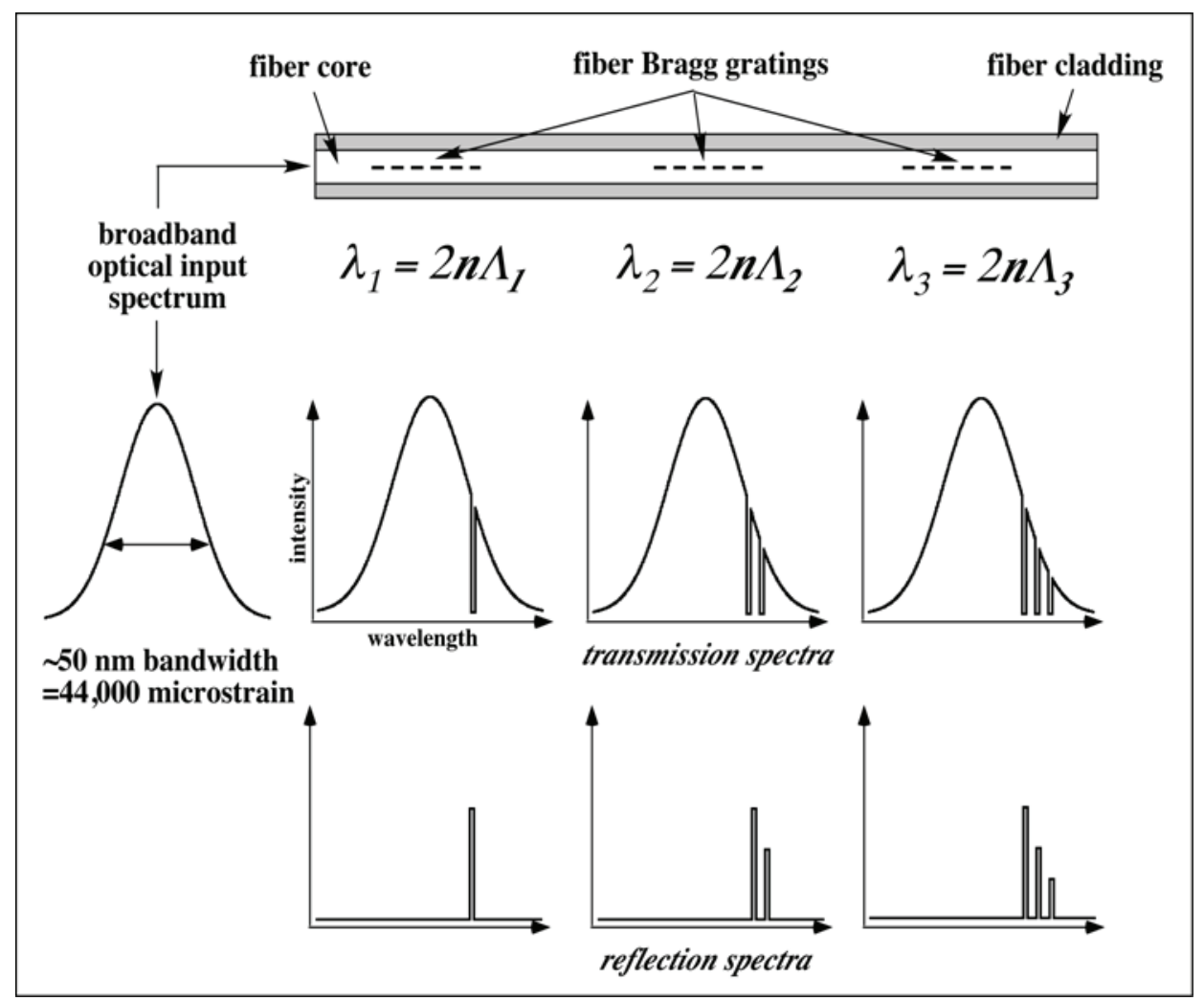

Figure 2.7. Bragg grating multiplexing.

Thus, tracking the individual reflected wavelength shifts allows a direct measurement of strain at the various locations. There are several architectures possible for doing this interrogation, but a dominant approach is shown in Figure 2.8. Here broadband light is inserted in an FBG array via a superluminescent diode, and the reflections from the array are coupled back through a Fabry-Perot filter. This device passes only a narrowband wavelength of light (designed to be commensurate with the FBG reflection width of $\sim 0.2 \mathrm{~nm}$ ) dependent upon the spacing between mirrors in the device. This spacing, and thus the passband, is controlled by applying a rapidly stepped voltage to a piezoelectric driver controlling the mirrors. The passed light signal is sent to a photodetector and differentiated; the zero-crossings of the differentiated signal correspond to the peak wavelengths of the reflected light, and correlation between the ramp voltage level and shifts in the zero-crossings results in obtaining the strain for each FBG sensor. The resolution of the voltage ramp and the spectral range of the filter primarily determine the optimal strain resolution, which has been demonstrated on the order of less than 1 microstrain; in some variations on this architecture, strain resolutions on the order of tens of nanostrain have been reported [15]. This resolution is several orders of magnitude better than the best foil resistive gage, but the costs of FBG systems (and specifically, the FBGs themselves, which are $\sim \$ 150$ per sensor) have limited deep market penetration. However, because FBG systems are insensitive to electromagnetic interference, do not create a spark source, are extremely lightweight and nonintrusive, and are highly multiplexible, many application areas, particularly in aerospace structural monitoring, are emerging. Furthermore, FBGs may be coupled with mechanical transducers to measure other fields such as acceleration, pressure, velocity, or temperature. 


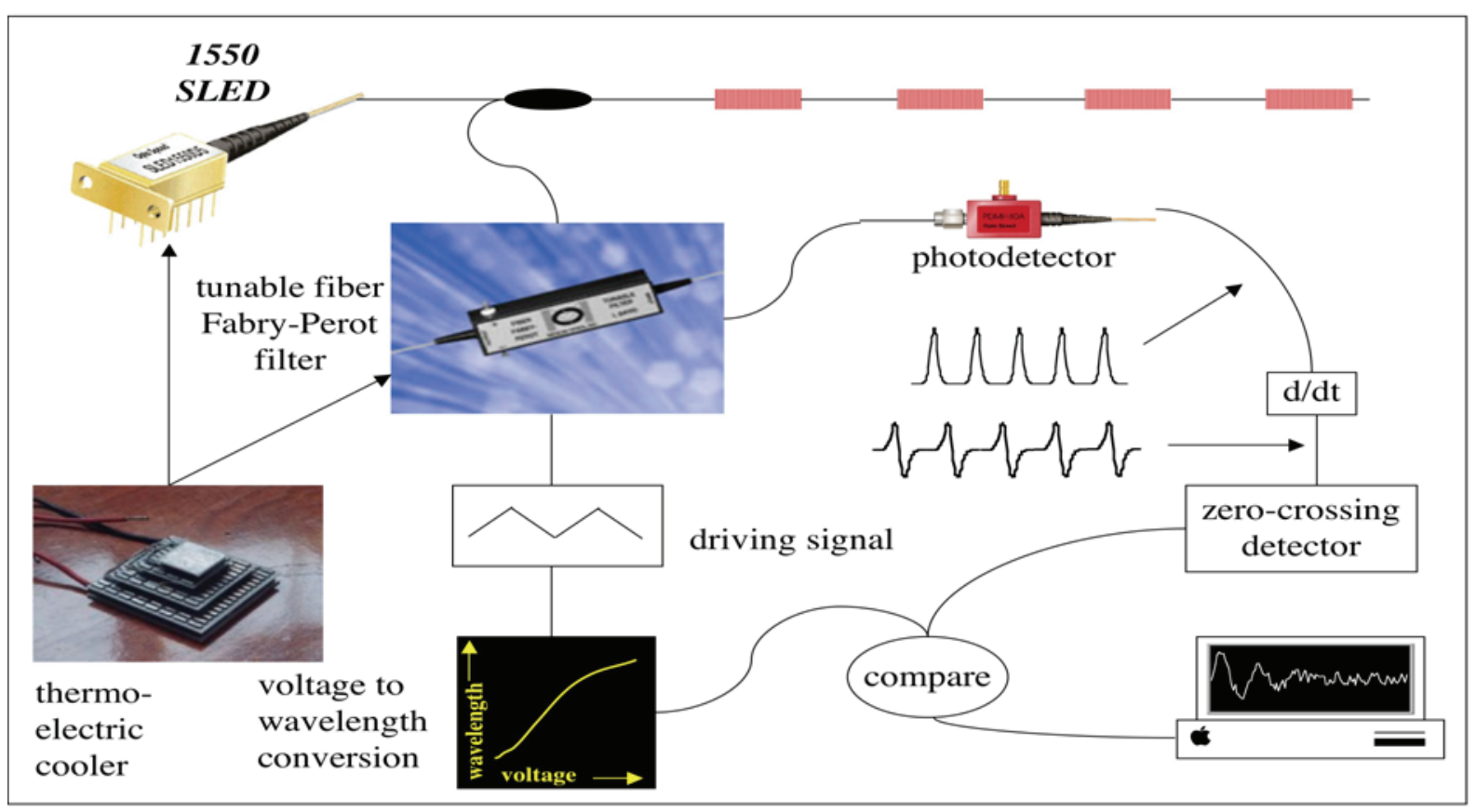

Figure 2.8. Fiber Bragg grating architecture using a tunable Fabry-Perot filter.

Power requirements for fiber-optic systems are usually larger than for conventional strain gage systems. The largest consumer in the fiber system shown in Figure 2.8, for example, is the thermoelectric cooler. This device is used to temperature-control the Fabry-Perot filter to ensure accurate voltage-to-wavelength conversion. While it is not required for some applications, in those for which it is required, it consumes energy at the rate of approximately 3-5 watts, depending on control demands imposed by the environment. The filter and SLED optical source used typically require power levels below $1 \mathrm{~W}$.

\subsubsection{Piezoelectric Sensor/Actuators}

The piezoelectric effect described above works both ways. The direct effect refers to a charge being produced when the material is strained, which is how the crystals used in the accelerometers work. However, the converse effect is also true: when a voltage is applied to the material, the material will deform proportionally to the applied potential difference, and this allows such materials to be used as both sensors (direct effect) and actuators (converse effect). These materials, usually ceramic- or polymer-based, may be fabricated into a variety of shapes amenable for various applications; some examples are shown in Figure 2.9.

Because these devices may be used in both modes, active sensing for SHM is possible. The devices discussed in sections 2.3.1-2.3.3 cannot be used as vibration excitation sources, but piezoelectric devices may be used to create local excitation to actively probe a structure. Arrays of these devices may be configured to induce local motion, and the same array is also used to measure the response. In the actuation mode, the free strain levels produced in typical piezoelectric materials such as lead zirconate titanate (PZT) are on the order of $0.1 \%-0.2 \%$, although newer relaxor ferroelectric crystals may produce strains on the order of $1 \%$ [16]. 


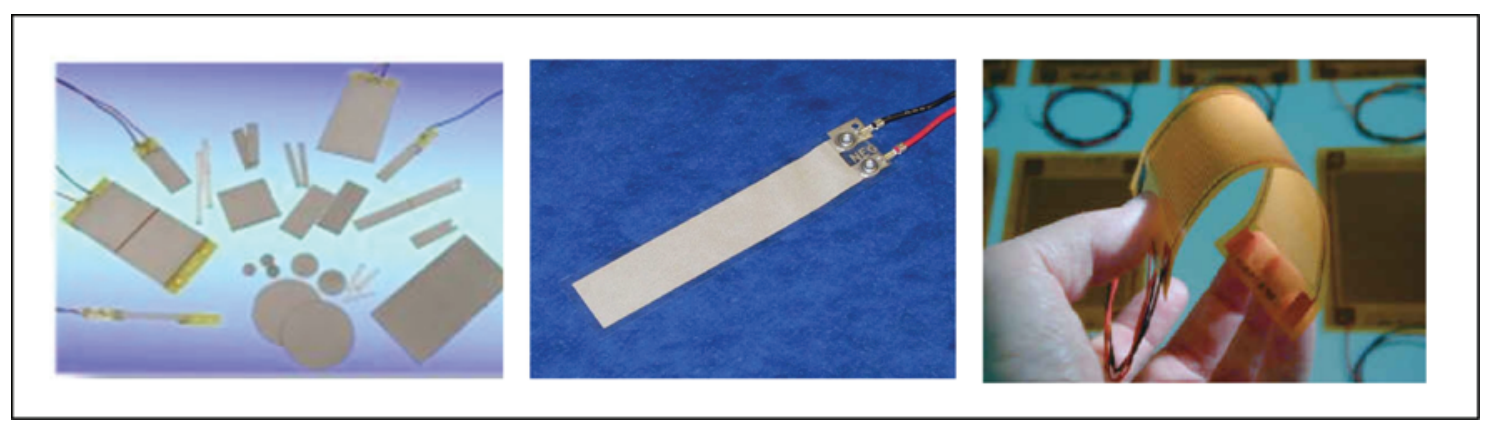

Figure 2.9. Some typical piezoelectric sensorlactuators from www.piezo.com (left), www.msiusa.com (center), and NASA Langley (right).

The performance of piezoelectric actuators can be characterized by their load lines (force/deflection curves). These curves are affected greatly by the inherent actuator design architecture, the material used, and the host system itself. For most applications, the voltage/output relationship is linear, and the optimal transfer of energy from the actuator to the host mechanical system is maximized when the stiffness of the actuator and the host are matched [17]. For a given applied voltage, the actuator displacement is reduced as the load is increased until the blocking force is reached at zero deflection. Addition of sensor elements in parallel or series modifies these curves, with a series arrangement compromising force for deflection and vice versa.

The electromechanical coupling property of piezoelectric materials allows one to design and deploy active-sensing systems in which the structure in question is locally excited by a known and repeatable input and the corresponding responses are measured by the same excitation source. The employment of a known input facilitates subsequent signal processing of the measured output data in SHM. Examples of using piezoelectric materials in the areas of active and local SHM sensing are Lamb wave propagations $[6,7,8,18,19]$ and the impedance-based structural health monitoring methods [5].

A typical SHM sensing system consists of a local computing processor, a signal conditioner, analog-to-digital (A/D) converters, and telemetry. An active-sensing system, however, requires additional components including a digital-to-analog (D/A) converter and a waveform generator. Because of the local-excitation and local-sensing nature, this system puts further demands on the use of much higher speed A/D converters, additional memory, and possibly multiplexers in order to control and manage a network of piezoelectric transducers. These extra components would inevitably demand more energy to complete an active-sensing SHM process.

In the passive sensing mode, piezoelectric transducers would consume much less energy, compared to accelerometers or strain gauges, because they do not require any electrical peripherals such as signal conditioning and amplification units. Any A/D converters could measure the charge output from a piezoelectric transducer, although this low power consumption characteristic will be modified if one needs to use charge amplifiers or voltage follower circuits to improve the signal-to-noise ratio, depending on the application or frequency range of interest.

For actuation, the average power requirement for the piezoelectric capacitive loading can be readily derived from lumped equivalent circuits as

$$
P=2 \pi f C V_{r m s}^{2}
$$


where $P$ is the average power requirement, $f$ is frequency, $C$ is the capacitive value of piezoelectric material, and $V_{r m s}$ is the RMS of the applied voltage. Under this relation, typical Lamb wave propagation approaches using a 6-mm disc-type piezoelectric patch (approximately $1 \mathrm{nF}$ ) would require around $300 \mathrm{~mW}$ of average power to launch $500 \mathrm{kHz} \mathrm{Lamb}$ waves, although the overall power demand will become much higher if one considers the peak power requirement or the energy consumed by a waveform generator with a D/A converter. It should be noted that this amount is dedicated to the actuation only, hence this level of power will be an additional energy requirement to the typical power consumption of passive sensing systems.

One of the primary ways to characterize actuation architectures is the amplification scheme employed (e.g., how the induced motion may be optimized and amplified): externally leveraged, internally leveraged, or frequency leveraged [20]. Externally leveraged actuators rely on an external mechanical component for actuation, and some examples of designs include flexurehinged, Cymbal, bimorph-based double-amplifier, flextensional, pyramid, and X-frame. This architecture usually achieves reasonable deflection amplification with linear behavior while retaining very stiff characteristics. Internally leveraged actuators differ from externally leveraged actuators in that they generate amplified strokes through the internal structure design without external components, with examples including (but not limited to) stack, bender, Rainbow, C-block, and Thunder. Because these designs rely on internal constraints, the stroke response is usually nonlinear, but it is greatly enhanced by the increased compliance of the design. Frequency-leveraged actuators utilize an alternating control signal to generate motion; the strain output is amplified by using the frequency performance of the actuator to move it in one direction in a series of small steps. Some designs include inchworm and ultrasonic motors. This architecture trades the speed of the piezoelectric material for (theoretically) infinite stroke, limited only by the actuator physical track and frictional effects.

\section{CURRENT SHM SENSOR NETWORK PARADIGMS}

Sensing systems for SHM consist of some or all of the following components:

- Transducers that convert changes in the field variable of interest (e.g., acceleration, strain, temperature) to changes in an electrical signal (e.g., voltage, impedance, resistance),

- Actuators that can be used to apply a prescribed input to the system (e.g., PZT tranducers bonded to the surface of a structure),

- Analog-to-digital (A/D) converters that transfer the analog electrical signal into a digital signal that can subsequently be processed on a computer. For the case where actuators are used, a digital-to-analog (D/A) converter will also be needed to change the prescribed digital signal to an analog voltage that can be used to control the actuator,

- Signal conditioning,

- Power,

- Telemetry,

- Processing, and

- Memory for data storage. 
The number of sensing systems available for SHM is enormous and these systems vary quite a bit depending upon the specific SHM activity. Two general types of SHM sensing systems are described below.

\subsection{Wired System}

Here wired SHM systems are defined as ones that telemeter data over direct wire connections from the transducers to the central data analysis facility, as shown schematically in Figure 3.1. In some cases the central data analysis facility is then connected to the internet such that the processed information can be monitored at a subsequent remote location. There is a wide variety of such systems. At one extreme are peak-strain or peak-acceleration sensing devices that notify the user when a certain threshold in the measured quantity has been exceeded. A more sophisticated system often used for condition monitoring of rotating machinery is a piezoelectric accelerometer with built-in charge amplifier connected directly to a hand-held, single-channel fast-Fourier-transform (FFT) analyzer. Here the central data storage and analysis facility is the hand-held FFT analyzer. Such systems cost on the order of a few thousand dollars. At the other extreme are custom designed systems with hundreds of data channels containing numerous types of sensors that cost on the order of multiple millions of dollars such as that deployed on the Tsing Ma bridge in China [21].

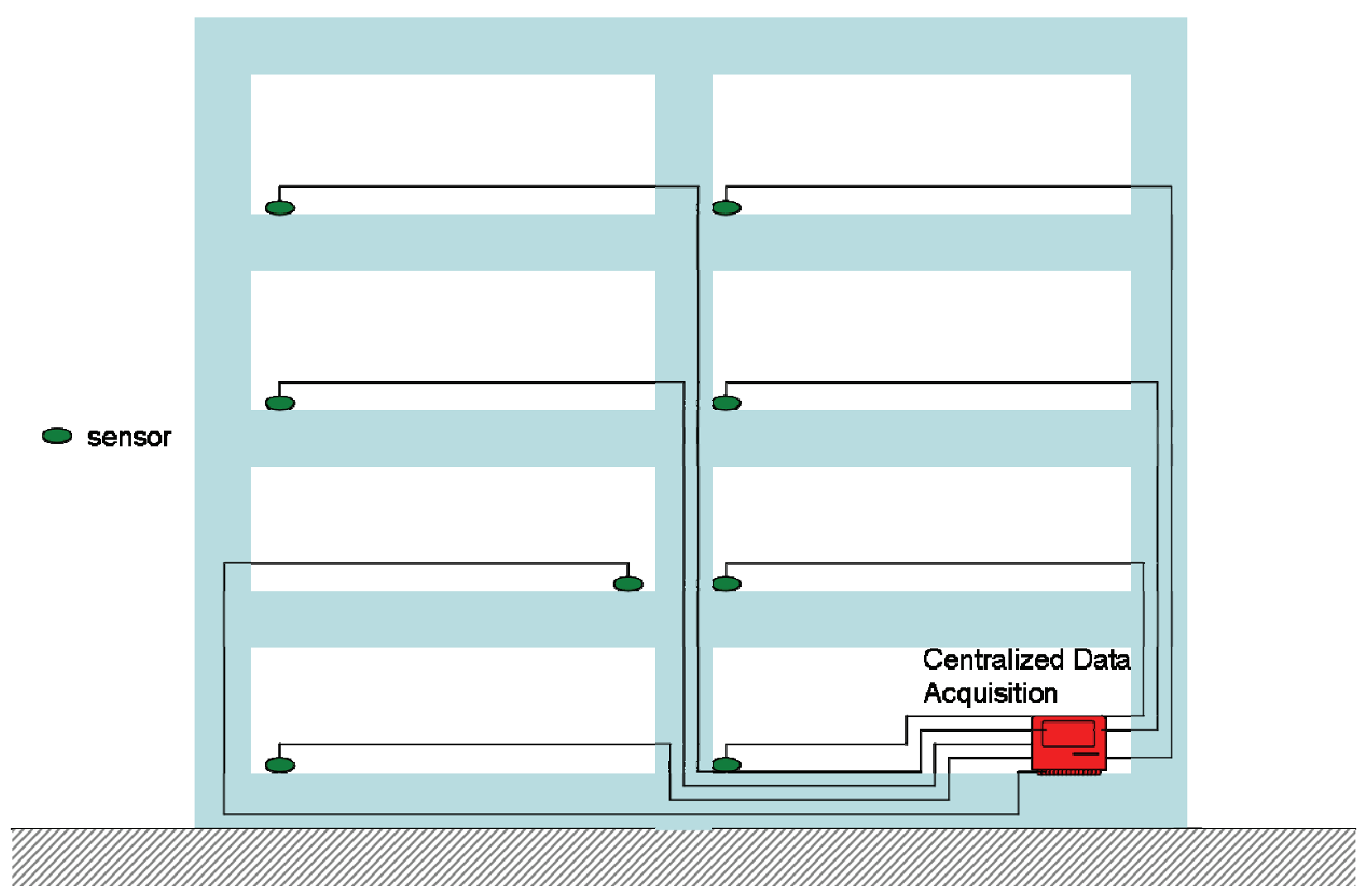

Figure 3.1. Conventional wired data acquisition system. 
There is a wide range of commercially available wired systems, some of which have been developed for general-purpose data acquisition and others that have been specifically developed for SHM applications. Those designed for general-purpose data acquisition typically can interface with a wide variety of transducers and also have the capability to drive actuators. The majority of these systems have integrated signal conditioning, data processing and data storage capabilities. The majority of these systems run off of $\mathrm{AC}$ power. Those designed to run off of batteries typically have a limited number of channels and they are limited in their ability to operate for long periods of time.

One wired system that has been specifically designed for SHM applications consists of an array of PZT patches embedded in Mylar sheet that is bonded to a structure [22]. The PZT patches can be used as either actuators or sensors. Damage is detected, located, and in some cases quantified by examining the attenuation of signals between different sensor-actuator pairs or by examining the characteristics of waves reflected from the damage. An accompanying PC is used for signal conditioning, A/D and D/A conversion, data analysis, and display of final results. The system, which runs on AC power, is shown in Figure 3.2.

\subsection{Wireless Transmission Systems}

Tanner et al. [23] adapted an SHM algorithm to the limitations of off-the-shelf wireless sensing and data processing hardware because of the focus toward a proof of concept rather than designing a field installable product. A wireless sensing system of "motes" running the TinyOS operating system developed at UC Berkeley was chosen because of their ready-made wireless communication capabilities. A mote consists of modular circuit boards integrating a sensor, microprocessor, A/D converter, and wireless transmitter, all of which run off of two AA batteries. A significant reduction in power consumption can be achieved by processing the data locally and only transmitting the results.

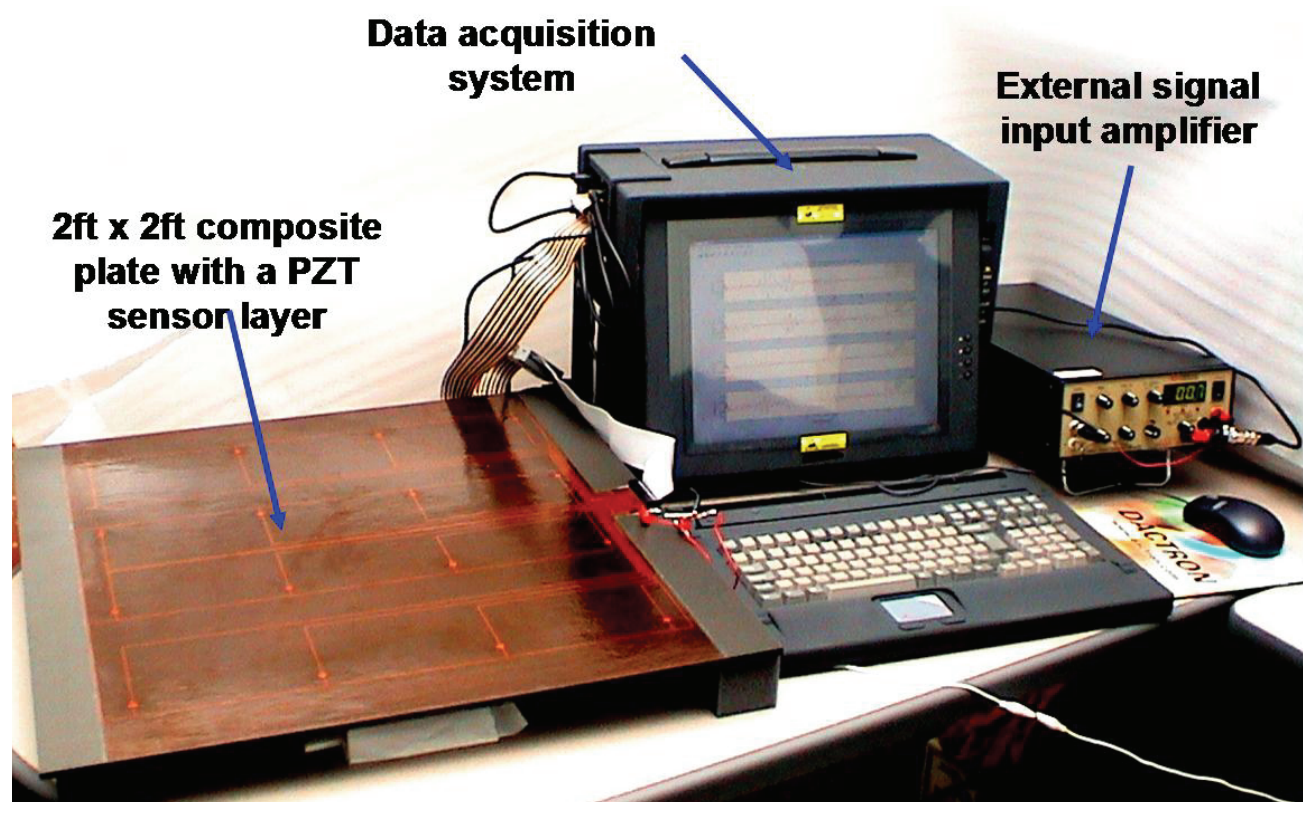

Figure 3.2. An active sensing system designed specifically for SHM. 
The core of the processor board is a 4-MHz ATMEL AVR 90LS8535 microprocessor with $8 \mathrm{~KB}$ of flash program memory and 512 bytes of RAM. A 10-bit A/D converter is included in this microprocessor. This converter is capable of sampling eight channels, but only by sequentially multiplexing the channels. A two-axis accelerometer mounted on a circuit board is integrated with the board as a sensing device. The processor board also contains three light emitting diodes (LED) and a short-range 916-MHz radio transmitter. Structural health monitoring algorithms were written on a PC and compiled into a binary image file that was downloaded into the flash program memory on the processing board. A binary result could then either be shown on the mote's LEDs or transmitted wirelessly to a base station. The system was demonstrated using a small portal structure with damage induced by loss of pre-load in a bolted joint. The tested mote system is shown in Figure 3.3. However, the processor proved to be very limited, allowing only the most rudimentary data interrogation algorithms to be implemented.

Lynch et al. [24] presented hardware for a wireless peer-to-peer SHM system. Using off-theshelf components, the authors couple sensing circuits and wireless transmission with a computational core, allowing decentralized collection, analysis, and broadcast of a structure's health. The final hardware platform includes two microcontrollers for data collection and computation connected to a spread spectrum wireless modem. The software is tightly integrated with the hardware and includes the wireless transmission module, the sensing module, and application module. The application module implements the time series based SHM algorithm. This integrated data interrogation process requires communication with a centralized sever to retrieve model coefficients. The object of the close integration of hardware and software with the dual microcontrollers strives for a power efficient design.

\section{Programming bay}

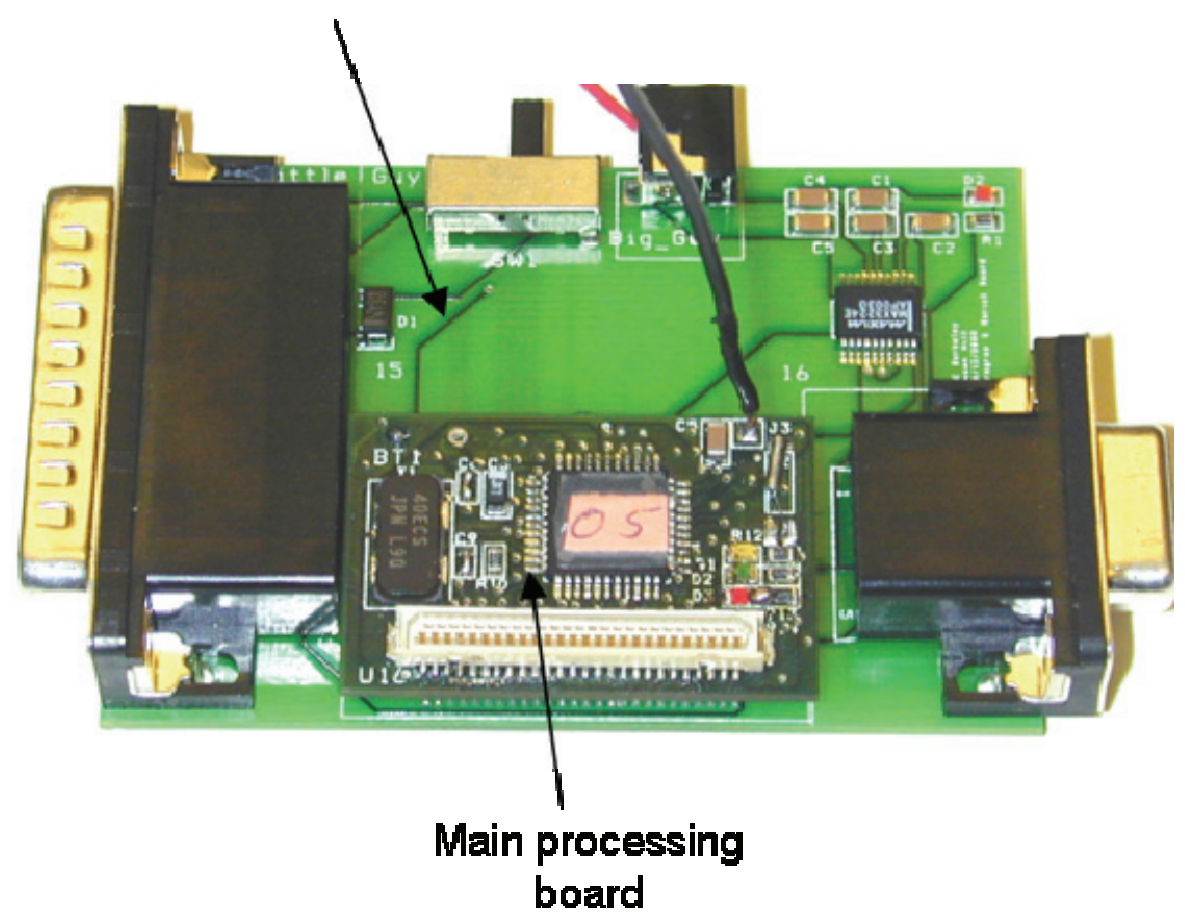

Figure 3.3. Mote sensing board mounted in programming bay. 
Spencer et al. [25] provide a state-of-the-art review of current "smart sensing" technologies that includes the compiled summaries of wireless work in the SHM field using small, integrated sensors and processor systems. A smart sensor is here defined as a sensing system with an embedded microprocessor and wireless communication. Many smart sensors covered in this article are still in the stage that simply sense and transmit data. The mote platform is discussed as an impetus for development of the next generation of SHM systems and a new generation of motes is also outlined. The authors also raised the issue that current smart sensing approaches scale poorly to systems with densely instrumented arrays of sensors that will be required for future SHM systems.

In order to develop a truly integrated SHM system, the data interrogation processes must be transferred to embedded software and hardware that incorporates sensing, processing, and the ability to return a result either locally or remotely. Most off-the-shelf solutions currently available or in development have a deficit in processing power that limits the complexity of the software and SHM process that can be implemented. Also, many integrated systems are inflexible because of tight integration between the embedded software, the hardware, and sensing.

To implement computationally intensive SHM processes, Farrar et al. selected a singleboard computer as a compact form of true processing power [26]. Also included in the integrated system is a digital signal processing board with six $\mathrm{A} / \mathrm{D}$ converters providing the interface to a variety of sensing modalities. Finally, a wireless network board is integrated to provide the ability for the system to relay structural information to a central host, across a network, or through local hardware. Figure 3.4 shows the prototype of this sensing system. Each of these hardware parts is built in a modular fashion and loosely coupled through a transmission control protocol over the internet. By implementing a common interface, changing or replacing a single component does not require a redesign of the entire system. By allowing processes developed in the Graphical Linking and Assembly of Syntax Structure (GLASS) client to be downloaded and run directly in the GLASS node software, this system becomes the first hardware solution where new processes can be created and loaded dynamically. This modular nature does not lead to the most power-optimized design, but instead achieves a flexible development platform that is used to find the most effective combination of algorithms and hardware for a specific SHM problem. Optimization for power is of secondary concern and will be the focus of follow-on efforts [26].

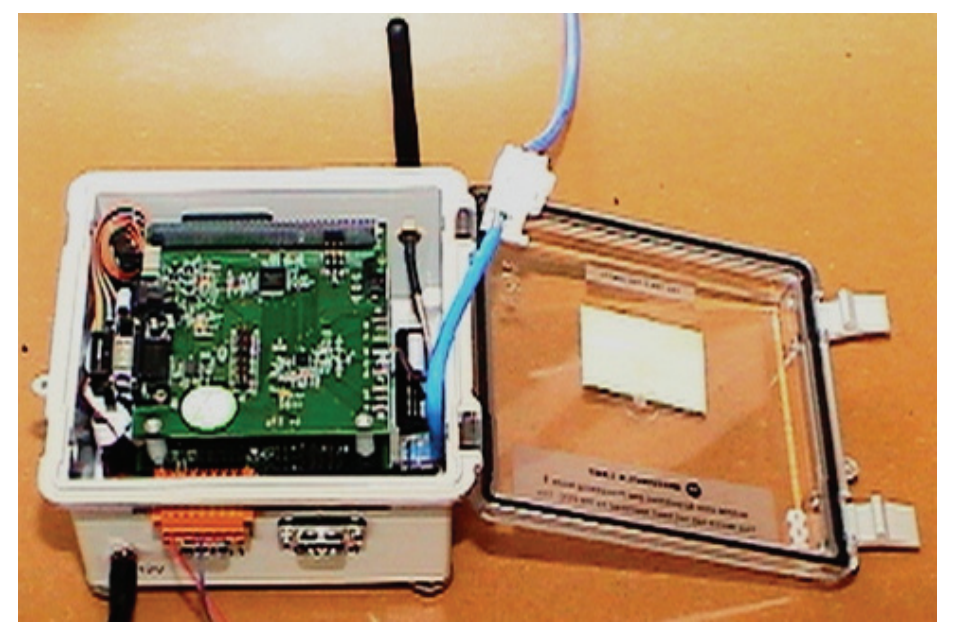

Figure 3.4. The wireless communication board displayed on the prototype SHM system. (Farrar et al. [26]) 


\subsection{Sensor Network Paradigms}

The sensor systems discussed in the previous section have led to three types of sensor network paradigms that are either currently being used for structural health monitoring or are the focus of current research efforts in this field. These paradigms are described below. Note that the illustrations of these systems show them applied to a building structure. However, these paradigms can be applied to a wide variety of aerospace, civil, and mechanical systems, and the building structure application is simply used for comparison purposes.

\subsubsection{Sensor Arrays Directly Connected to Central Processing Hardware}

Figure 3.1 shows a sensor network directly connected to the central processing hardware. Such a system is the most common one used for structural health monitoring studies. The advantage of this system is the wide variety of commercially available off-the-shelf systems that can be used for this type of monitoring and the wide variety of transducers that can typically be interfaced with such a system. For SHM applications, these systems have been used in both a passive and active sensing manner. A limitation of such systems is that they are difficult to deploy in a retrofit mode because they usually require $\mathrm{AC}$ power, which is not always available. Also, these systems are one-point failure sensitive as one wire can be as long as a few hundred meters. In addition, the deployment of such systems can be challenging with potentially over $75 \%$ of the installation time attributed to the installation of system wires and cables for larger scale structures such as those used for long span bridges [27]. Furthermore, experience with fielddeployed systems has shown that the wires can be costly to maintain because of general environmental degradation and damage caused by things such as rodents and vandals.

\subsubsection{Decentralized Sensing and Processing with Hopping Connection}

The integration of wireless communication technologies into SHM methods has been widely investigated in order to overcome the limitations of wired sensing networks. Wireless communication can remedy the cabling problem of the traditional monitoring system and significantly reduce the maintenance cost. The schematic of the decentralized wireless monitoring system, which is summarized in detail by Spencer et al. [25] and Lynch and Loh [28], is shown in Figure 3.5.

From the large-scale SHM practice, however, several very serious issues arise with the current design and deployment scheme of the decentralized wireless sensing networks [25, 27]. First, the current wireless sensing design usually adopts ad-hoc networking and hopping that results in a problem referred to as data collision. Data collision is a phenomenon that results from a network device receiving several simultaneous requests to store or retrieve data from other devices on the network. With increasing numbers of sensors, a sensor node located close to the base station would experience tremendous data transmission, possibly resulting in a significant bottleneck. Because the workload of each sensor node cannot be evenly distributed, the chances of data collision increase with expansion of the sensing networks. In addition, this decentralized wireless sensing network scales very poorly in active-sensing system deployment. Because active sensors can serve as actuators as well as sensors, the time synchronization between multiple sensor/actuator units will be a challenging task. Furthermore, the cost of implementing such a system into a large-scale structure is extremely prohibitive for increasing numbers of active sensors. The cost of current decentralized wireless sensor nodes is at least two orders of magnitude greater than that of an active sensor, which can usually be obtained for less than five 


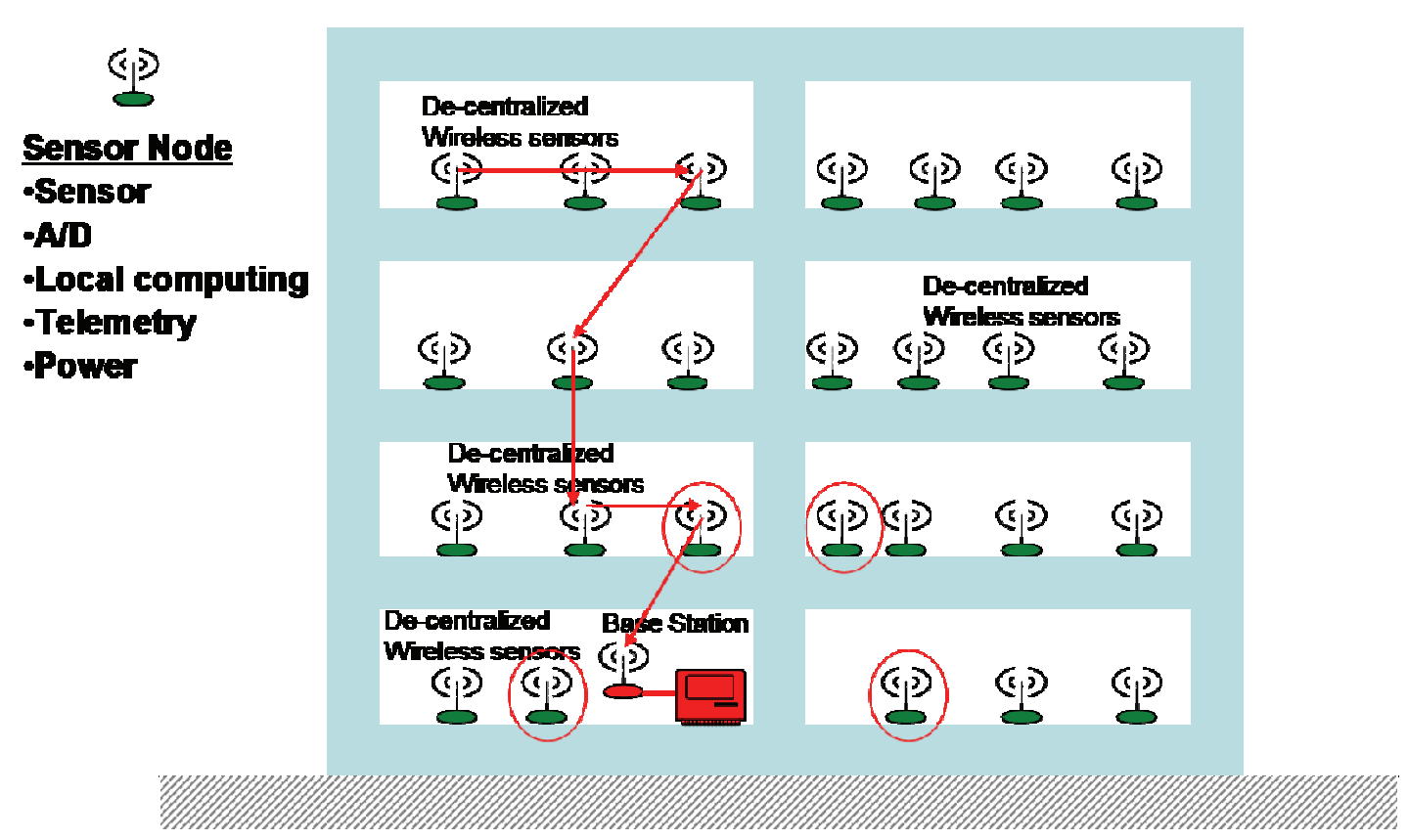

Figure 3.5. Decentralized wireless SHM system employing hopping communications protocol.

dollars. Because of the processor scheduling or sharing, the use of multiple channels with one sensor node would reduce the sampling rate, which provides neither a practical nor equitable solution for active-sensing techniques that typically adopt higher frequency ranges. Therefore, in real-life applications, the current design scheme could turn out to be a very expensive operation.

\subsubsection{Decentralized Active Sensing and Processing with Hybrid Connection}

The hybrid connection network advantageously combines the previous two networks, as illustrated in Figure 3.6. At the first level, several sensors are connected to a relay-based piece of hardware, which can serve as both a multiplexer and general-purpose signal router, shown in Figure 3.6 as a black box. This device will manage the distributed sensing network, control the modes of sensing and actuation, and multiplex the measured signals. The device can also be expandable by means of daisy-chaining. At the next level, multiple pieces of this hardware are linked to a decentralized data control and processing station. This control station is equipped with data acquisition boards, on-board computing processors, and wireless telemetry, which is similar to the architecture of current decentralized wireless sensors. This device will perform duties of a relay-based hardware control system, including data acquisition, local computing, and transmission of the necessary results of the computation to the central system. At the highest level, multiple data processing stations are linked to a central monitoring station that delivers a damage report back to the user. Hierarchal in nature, this sensing network can efficiently interrogate large numbers of distributed sensors and active sensors, while maintaining an excellent sensor-cost ratio because only a small number of data acquisition and telemetry units is necessary. This hierarchal sensing network is especially suitable for active-sensing SHM techniques, and it is being substantially investigated by Dove et al. [29]. In their study, the expandability or the sensing network was of the utmost importance for significantly larger numbers of active sensors, as the number of channels on a decentralized wireless sensor is limited because of the processor sharing and scheduling. The prototype of the "Blackbox" is illustrated in Figure 3.7. 

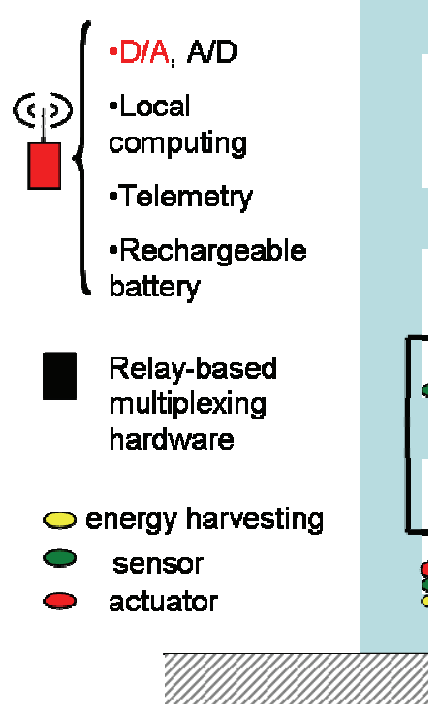

Figure 3.6. Decentralized wireless, active SHM system with energy harvesting and hybrid connection

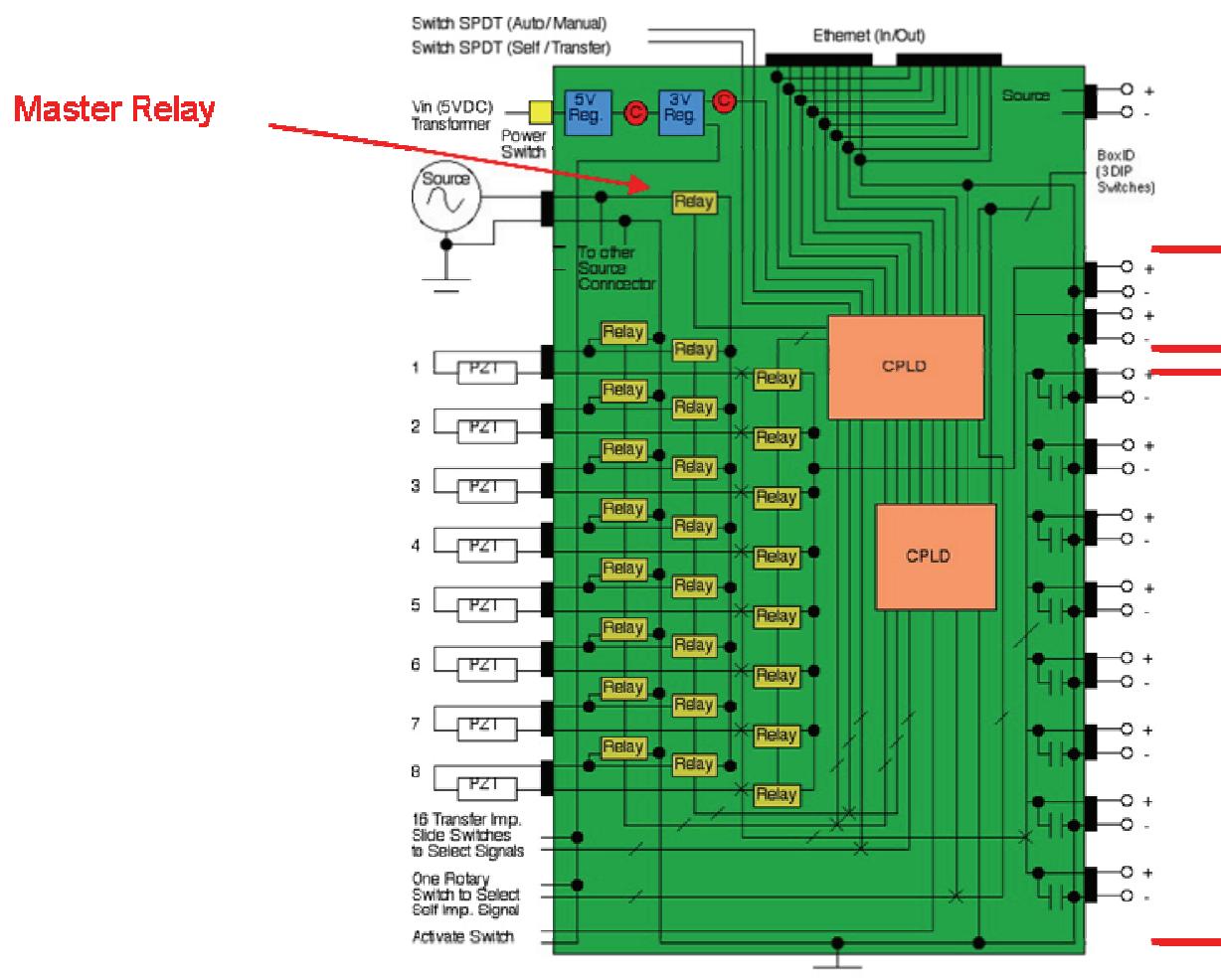

Multiplexed Channels

Direct Output Channels

Figure 3.7. Relay-based hardware with optional manual controls included. 


\subsection{Future Sensing Network Paradigms}

The sensing network paradigms described in the previous section have one characteristic in common. The sensing system and associated power sources are installed at fixed locations on the structural system. As stated, the deployment of such sensing systems can be costly and the power source may not be always available. A new, efficient future sensing network is currently being investigated by UCSD and LANL researchers by integrating active radio-frequency identification (RFID) sensing technology and remote interrogation platforms based on either robots or unmanned aerial vehicles (UAVs) to assess damage in structural systems. This approach involves using an unmanned mobile host node (delivered via UAV or robot) to generate an RF signal near sensors that have been embedded on the structure. The sensors measure the desired response (impedance, strain, etc.) at critical areas on the structure and transmit the signal back to the mobile host again via the RF communications. This "wireless" communications capability draws power from the RF energy transmitted between the host and sensor nodes and uses it to both power the sensing circuit and transmit the signal back to the host (see Figure 3.8). The host itself, with embedded computing circuitry, may cost on the order of $\$ 1000$, but only one such host will be needed to interrogate an entire sensor array placed on the structure. This research takes traditional sensing networks to the next level, as the mobile hosts (such as UAVs) will fly to a known critical infrastructure based upon a GPS locator, deliver required power, and then begin to perform an inspection without human intervention. The mobile hosts will search for the sensors on the structure and gather critical data needed to perform the structural health evaluation. This project will tailor a specialized UAV made of lightweight composite materials that will weigh less than $55 \mathrm{lbs}$ in order get FAA approval for flying in populated air spaces and be able to access tight spaces. This integrated technology will be directly applicable to rapid structural condition assessment of buildings and bridges after an earthquake. Also, this technology may be adapted and applied to damage detection in a variety of other civilian and defense-related structures such as pipelines, naval vessels, hazardous waste disposal containers, and commercial aircraft.

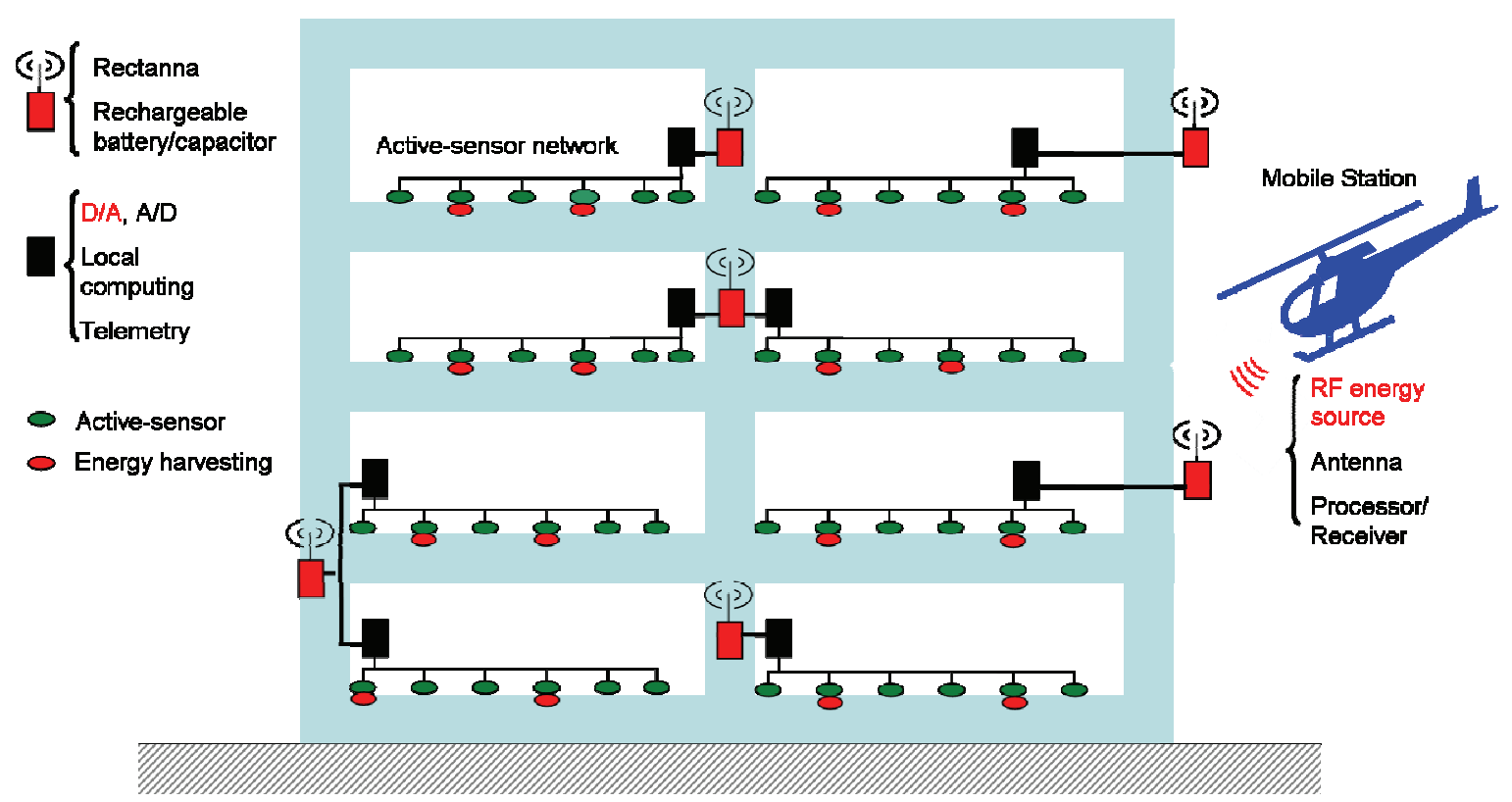

Figure 3.8. Active sensing network that includes energy harvesting and that is interrogated by an unmanned robotic vehicle. 


\subsection{Practical Implementation Issues for SHM Sensing Networks}

Major concerns in the current sensing network development community are long-term reliability and sources of power. Other concerns are the abilities of the sensing systems to capture local and system-level responses, that is, the need to capture responses on widely varying length and time scales and to archive data in a consistent, retrievable manner for long-term analysis. These challenges are nontrivial because of the tendency for each technical discipline to work more or less in isolation. Therefore, an integrated systems engineering approach to the damage detection process and regular, well-defined routes of information dissemination are essential. The subsequent portions of this section will address specific sensing system issues associated with SHM.

\subsection{Sensor Properties}

One of the major challenges of defining sensor properties is that these properties need to be defined a priori and typically cannot be changed easily once a sensor system is in place. These properties of sensors include bandwidth, sensitivity (dynamic range), number, location, stability, reliability, cost, telemetry, etc. To address this challenge, a significantly coupled analytical and experimental approach to the sensor system deployment should be used, in contrast to the current ad hoc procedures used for most current damage detection studies. This strategy should yield considerable improvements. First, critical failure modes of the system can be well defined and, to some extent, quantified using high-fidelity numerical simulations or from previous experiences before the sensing system is designed. The high-fidelity numerical simulations/experiences can be used to define the required bandwidth, sensitivity, sensor location, and sensor number. Additional sensing requirements can also be ascertained if changing operational and environmental conditions are included in the models so as to determine how these conditions affect the damage detection process.

Another potential level of integration between modeling and sensing resides in the integration of software and hardware components. Once the actuation and sensing capability has been selected, their location has been optimized, and the specification of the data acquisition system have been met, it may be advantageous to integrate model output and sensing information as much as possible. For example, surrogate models can be programmed on local digital signal processing (DSP) chips and their predictions can be compared to sensor output in real time. One obvious benefit would be to minimize the amount of communication by integrating the analysis capability with real-time sensing. In an integrated approach, features can be extracted from sensing information and numerical simulation. Test-analysis comparison and parameter estimation can then be performed locally, which would greatly increase the efficiency of damage detection.

\subsection{Sensor Calibration and Ruggedness}

Most sensors are calibrated at a specialized calibration facility. This type of calibration is expected to endure but to be supplemented by self-checking and self-calibrating sensors. Calibration raises several important issues. It is not clear just what forms of calibration are essential and what are superfluous. Some measurements are acceptable with $20 \%$ error, especially if sensor-to-sensor comparisons are accurate within a few percent. In other scenarios, 
absolute accuracies better than $1 \%$ are required. The calibration community needs to address these issues, including both precision (for example, how to calibrate a 32-bit digitizer over its entire dynamic range) and flexibility (calibration of a precise sensor vs calibration of a coarse sensor).

Confidence and robustness in the sensors are prime considerations for SHM. If this part of the system is compromised, the overall confidence in the system performance is undermined. For sensors implemented for SHM, several durability considerations emerge:

- The nontrivial problem of sensor selection for extreme environments, e.g., in service turbine blades.

- Sensors being less reliable than the part. For example, reliable parts may have failure rates of 1 in 100,000 over several years' time. Sensors are often small, complex assemblies, so sensors may fail more often than the part sensed. Loss of sensor signal then falsely indicates part failure, not sensor failure.

- Sensors may fail through outright sensor destruction while the part sensed endures.

False indications of damage or damage precursors are extremely undesirable. If this occurs often, the sensor is either overtly or implicitly ignored. Recently several studies have focused on issues of sensor validation $[30,31]$.

\subsection{Multiscale Sensing}

Depending on the size and location of the structural damage and the loads applied to the system, the adverse effects of the damage can be immediate or it may take some time before the system's performance is altered. In terms of length scales, all damage begins at the material level and then, under appropriate loading conditions, progresses to component and system level damage at various rates. In terms of time scales, damage can accumulate incrementally over long periods of time, such as that associated with fatigue or corrosion damage accumulation. Damage can also occur on much shorter time scales as a result of scheduled discrete events such as aircraft landings and from unscheduled discrete events such as enemy fire on a military vehicle. Therefore, the most fundamental issue that must be addressed when developing a sensing system for SHM is the need to capture the structural response on widely varying length and time scales. Sensors with a high frequency range tend to be more sensitive to local response and therefore to damage. This requires a sensor with a large bandwidth. Typically, as the bandwidth goes up, the sensitivity goes down. Also, it is harder to excite higher frequencies, thus the excitation needs to be very local as is possible with piezoelectric actuators.

The sensing systems that are able to capture the responses over varying length and time scales have not been substantially investigated by researchers, although it is quite possible to use the same piezoelectric patches in both active (high frequency) and passive (lower-order global) modes. When used in the passive mode, the sensors detect strain resulting from ambient loading conditions and can be used to monitor the global response of a system. In the active mode the same sensors can be used to detect and locate damage on a local level using relatively higher frequency ranges. 


\subsection{Power Consideration}

A major consideration in using a dense sensor array is the problem of providing power to the sensors. This demand leads to the concept of "information as a form of energy." Deriving information costs energy. If the only way to provide power is by direct connections, then the need for wireless protocols is eliminated, as the cabled power link can also be used for the transmission of data. Hence, the development of micropower generators is a key factor for the development of the hardware if wireless communication is to be used. A possible solution to the problem of localized power generation are technologies that enable harvesting ambient energy to power the instrumentation [32]. Forms of energy that may be harvested include thermal, vibration, acoustic, and solar. Although this is new technology, the overriding consideration of reliability still exists, as it does with any monitoring system. With two-way communication capability, the local sensing and processing units also can put themselves "to sleep" and go "off-line" for energy conservation and they can resume operation when a "wake-up" signal is broadcast.

\section{ENERGY DEMANDS ASSOCIATED WITH SHM SENSING SYSTEMS}

\subsection{Introduction}

Embedded system design is characterized by a tradeoff between a need for good performance and low power consumption. Proliferation of wireless sensing devices has stressed even more the need for energy minimization as battery capacity has improved very slowly (by a factor of 2 to 4 over the last 30 years), while computational demands have drastically increased over the same time frame, as shown in Figure 4.1.

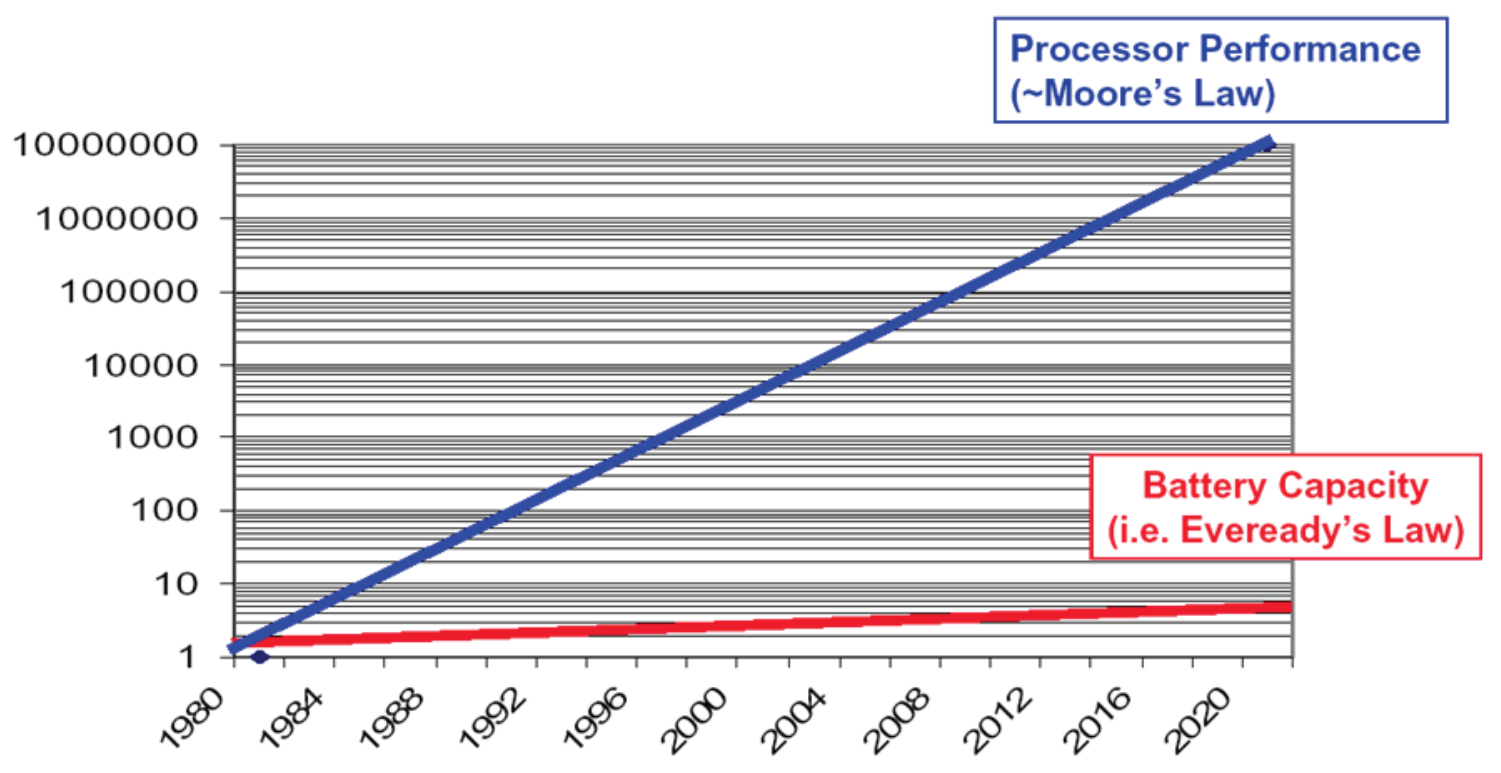

Figure 4.1. Battery capacity vs processor performance. 
Since the introduction of wireless computing, the demands on battery lifetime have grown even more. In fact, in most of today's embedded sensing devices, the wireless connectivity consumes a large fraction of the overall energy consumption. Figure 4.2 shows a power consumption breakdown for a small sensor node (top of the figure) and a larger embedded device based on a Strong ARM processor $(200 \mathrm{MHz})$ coupled with a wireless local area network (WLAN) for communication [33]. On small sensor nodes, as much as $90 \%$ of the overall system power consumption can go to wireless communication, while on the larger devices, such as the one shown at the bottom of Figure 4.2, the wireless takes approximately $50 \%$ of the overall power budget. In both cases, the second most power hungry device is the processor. Therefore, in order to achieve long battery lifetimes, optimization of both computing and communication energy consumption is critically important.

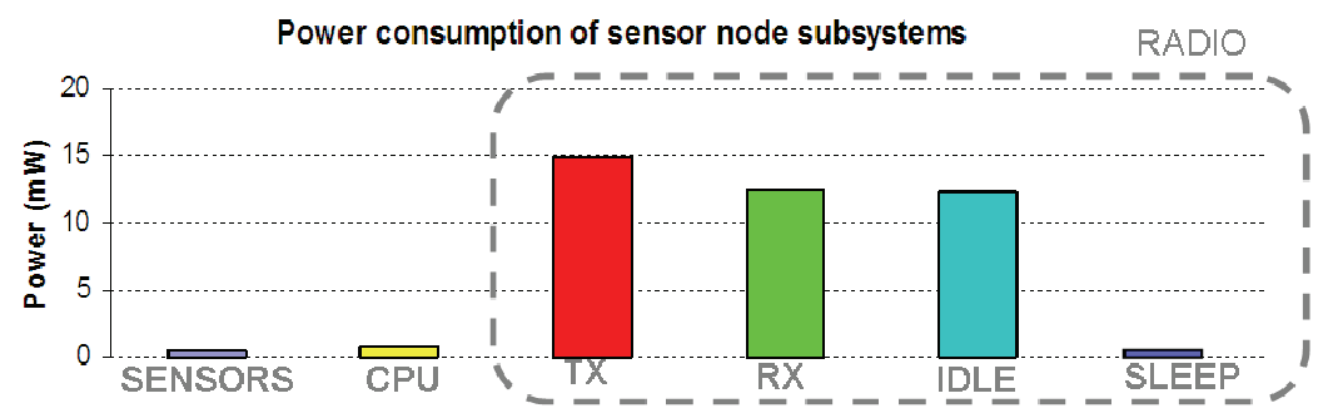

Energy breakdown for audio: Strong ARM CPU and WLAN

\section{Encode Decode}

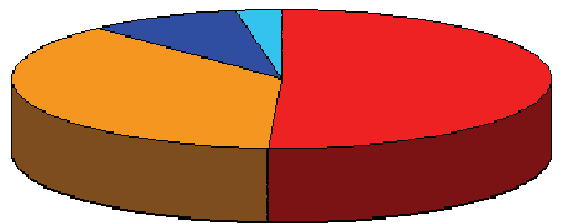

Receive
Transmit
Energy breakdown for video: Strong ARM CPU and WLAN

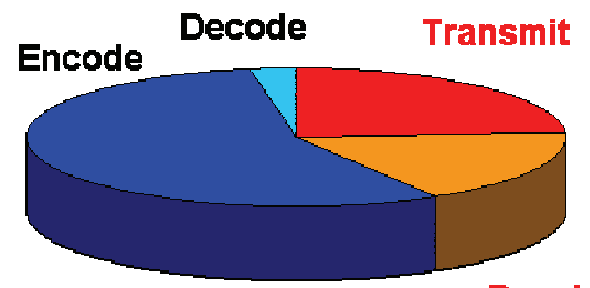

Receive

Figure 4.2. Power consumption of two different embedded system designs.

Better low-power circuit design techniques have helped to lower the power consumption $[34,35,36]$. On the other hand, managing power dissipation at higher levels can considerably decrease energy requirements and thus increase battery lifetime and lower packaging and cooling costs [37, 38]. Two different approaches for lowering the power consumption at the system level have been proposed: dynamic voltage scaling, primarily targeted at the processing elements, and dynamic power management, which can be applied to all system components.

$$
\begin{aligned}
& P_{d y n} \propto f V_{d d}^{2} \\
& f \propto\left(V_{d d}-V_{\text {treshold }}\right)^{2} / V_{d d}
\end{aligned}
$$


Embedded sensing systems are designed to be able to deliver peak performance when needed, but most of the time their components operate at utilizations less than $100 \%$. One way of lowering the power consumption is by slowing down the execution, and, when appropriate, also lowering the component's voltage of operation. This power reduction is done with dynamic voltage scaling (DVS) algorithms. The primary motivation comes from the observation that dynamic power consumption, $P_{d y n}$, is directly proportional to the frequency of operation, $f$, and the square of the supply voltage, $V_{d d}^{2}$ [see Equation (1)]. Frequency, in turn, is a linear function of $V_{d d}$, [see Equation (2)], so decreasing the voltage results in a cubic decrease in the power consumption. Clearly, decreasing the voltage also lowers the frequency of operation, which, in turn, lowers the performance of the design. Figure 4.3 shows the effect of DVS on power and performance of a processor. Instead of having longer idle period, the central processing unit $(\mathrm{CPU})$ is slowed down to the point where it completes the task in time for the arrival of the next processing request while at the same time saving quite a bit of energy. DVS algorithms are typically implemented at the level of an operating system (OS) scheduler. Thus, instead of using only performance as a way to guide decisions on which task should be scheduled at what point in time, now reduction of energy is also considered. There have been a number of voltage scaling techniques proposed for real-time systems. Early work typically assumed that the tasks run at their worst-case execution time (WCET), while the later research work relaxes this assumption and suggests a number of heuristics for prediction of task execution time.

In contrast to DVS, system-level dynamic power management (DPM) decreases the energy consumption by selectively placing idle components into lower power states. DVS can only be applied to CPUs, while DPM can be used to reduce the energy consumption of wireless communication, CPUs, and all other components that have low power states. While slowing down the CPU with DVS can provide quite a bit of power savings, applying DPM typically increases the savings by at least a factor of 10 and in many systems by significantly more than that. On the other hand, changing processor speed happens relatively quickly, while the transitions in and out of sleep states can be quite costly in terms of both energy and performance. Figure 4.4 shows both power and performance overheads incurred during the transition. At a minimum the device needs to stay in the low-power state for long enough (defined as the breakeven time, $T_{B E}$ ) to recuperate the cost of transitioning. The break-even time, as defined in Equation (3), is a function of the power consumption in the active state, $P_{\text {on }}$, the amount of power consumed in the low power state, $P_{\text {sleep }}$, and the cost of the transition in terms of both time, $T_{t r}$, and power, $P_{p r}$.

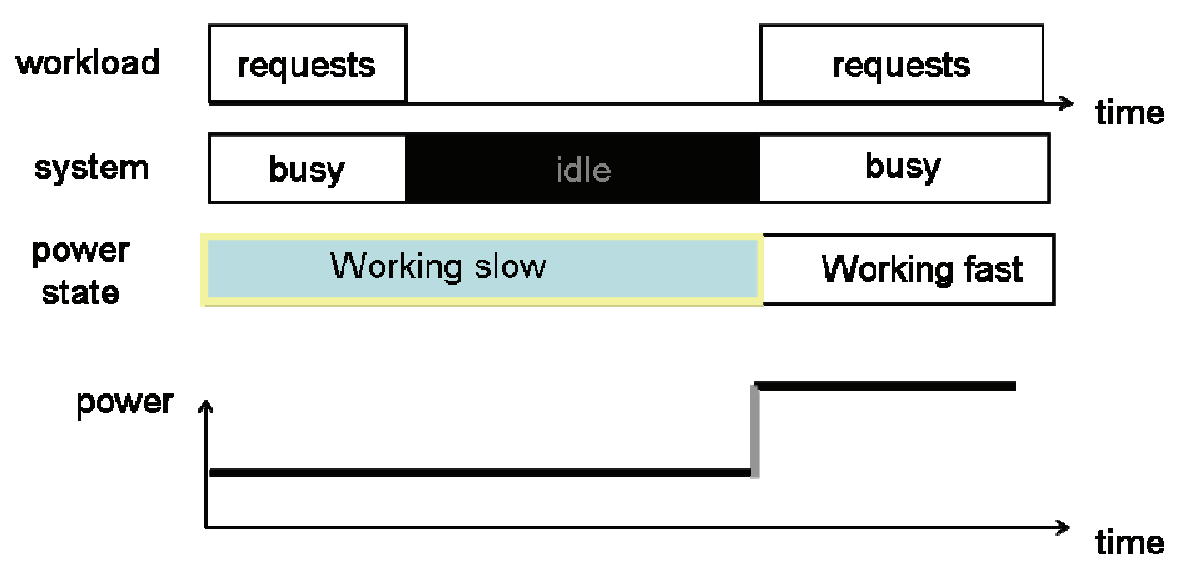

Figure 4.3. Dynamic voltage scaling for a single processor. 


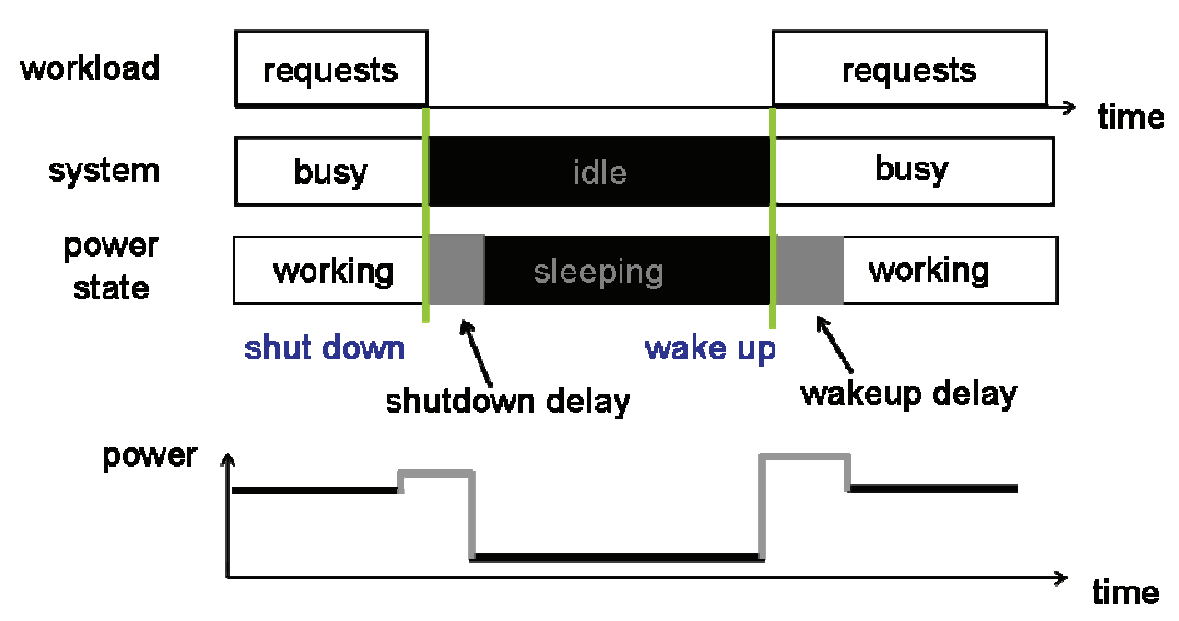

Figure 4.4. Dynamic power management for a single device.

$$
T_{B E}=T_{t r}+T_{t r} \frac{P_{t r}-P_{o n}}{P_{o n}-P_{\text {sleep }}}
$$

If it were possible to predict ahead of time the exact length of each idle period, the ideal power management policy would place a device in the sleep state only when an idle period would be longer than the break-even time. Unfortunately, in most real systems such perfect prediction of idle periods is not possible. As a result, one of the primary tasks DPM algorithms have is to predict when the idle period will be long enough to amortize the cost of transition to a low power state and to select the state to transition to. Three classes of policies can be defined: timeout based, predictive, and stochastic. Timeout policy is implemented in most operating systems. The drawback of this policy is that it wastes power while waiting for the timeout to expire. Predictive policies developed for interactive terminals $[39,40]$ force the transition to a low power state as soon as a component becomes idle if the predictor estimates that the idle period will last long enough. An incorrect estimate can cause both performance and energy penalties. Both timeout and predictive policies are heuristic in nature, and thus do not guarantee optimal results. In contrast, approaches based on stochastic models can guarantee optimal results. Stochastic models use distributions to describe the times between arrivals of user requests (interarrival times), the length of time it takes for a device to service a user's request, and the time it takes for the device to transition between power states. The optimality of stochastic approaches depends on the accuracy of the system model and the algorithm used to compute the solution.

Finally, much recent work has looked at combining DVS and DPM into a single power management implementation. Shorter idle periods are more amenable to DVS, while longer ones are more appropriate for DPM. Thus, a combination of the two approaches is needed for optimal results. The rest of this chapter provides an overview of state-of-the-art dynamic power management and dynamic voltage scaling algorithms that can be used to reduce the power consumption of processing and communication in wireless sensing devices. 


\subsection{Dynamic Power Management}

Dynamic power management (DPM) techniques selectively place system components into low-power states when they are idle. A managed-power system can be modeled as a power state machine, where each state is characterized by the power consumption and the performance. In addition, state transitions have power and delay costs. Usually, lower power consumption also implies lower performance and longer transition delay. When a component is placed into a lowpower state, such as a sleep state, it is unavailable for the time period spent there, as well as during the transition time between the states. The transitions between states are controlled by commands issued by a power manager (PM) that observes the workload of the system and decides when and how to force power state transitions. The power manager makes state transition decisions according to the power management policy. The choice of the policy that minimizes power under performance constraints (or maximizes performance under power constraints) is a constrained optimization problem.

The system model for power management therefore consists of three components: the user, the device, and the queue, as shown in Figure 4.5. The user, or the application that accesses each device by sending requests, can be modeled with a request interarrival time distribution. When one or more requests arrive, the user is said to be in the active state, otherwise it is in the idle state. Figure 4.5 shows three different power states for the device: active, idle, and sleep. Often the device will have multiple active states, which can be differentiated by the frequency and voltage of operation. Similarly, there can be multiple inactive states-both idle, each potentially corresponding to a specific frequency of operation in the active state (e.g., XScale CPU), and sleep states. Service time distribution describes the behavior of the device in the active state. When the device is in either the idle or the sleep state, it does not service any requests. Typically, the transition to the active state is shorter from the idle state, but the sleep state has lower power consumption. The transition distribution models the time taken by the device to transition between its power states. The queue models a buffer associated with each device. The combination of interarrival time distribution (incoming requests to the buffer) and service time distribution (requests leaving the buffer) can be used to fully characterize the behavior of the queue.

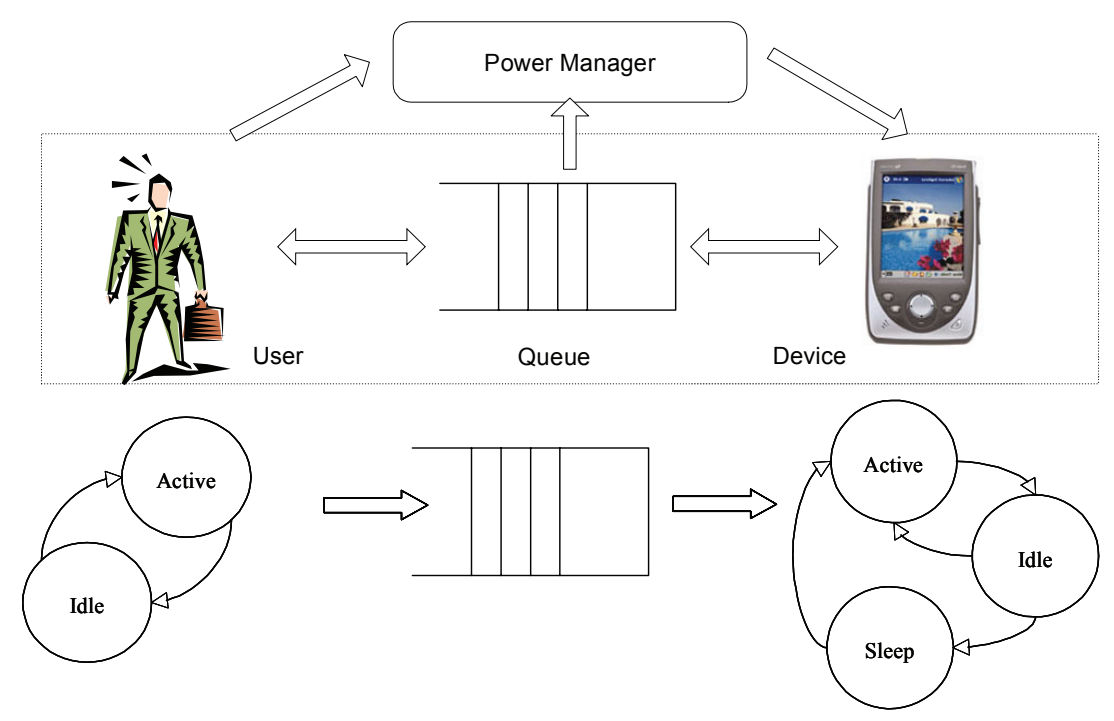

Figure 4.5. System model. 
The power manager's job can consist of a number of tasks: (1) tracking and modeling of incoming service requests with a goal of predicting when longer idle periods occur; (2) traffic shaping-buffering the traffic so larger idle periods are created that enable the device to stay asleep for a longer time period; (3) deciding when and to what sleep state a device should transition to; and (4) making a decision as to when a device should wake up to process requests. In this section we provide an overview of DPM policies that address various combinations of these tasks.

\subsection{Heuristic Policies}

Most commercial power management implementations focus only on deciding when a device should go to sleep. The cost of transition between the active and the sleep state typically determines how aggressive a policy can be. When the cost is reasonably low, or is at least not perceivable by the users, then policies transition a device to sleep as soon as it becomes idle. For example, " $\mu$ Sleep" has been introduced as a way of reducing the idle power consumption of HP's IPAQ platform [41]. IBM's wristwatch enters standby state as soon as it becomes idle [42]. Intel's QuickStart technology puts a processor to sleep between the keystrokes. In all three cases the cost of waking up is not perceivable by the user.

When the cost of transition to a low power state is significant, then commercial applications typically implement policies based on timeout. For example, HP's IPAQ uses a timeout value to decide when the display should be dimmed. The timeout value is usually either fixed at design time or can be set by a user.

\subsubsection{Timeout Policies}

Timeout policies assume that if the incoming workload has an idle period that is longer than some timeout value $T_{t o}$, then there is a very high likelihood that the idle period will be long enough to justify going to a sleep state. Therefore, the total length of the idle time needs to be longer than $T_{t o}+T_{b e}$. Timeout policies waste energy during the timeout period, as they keep a device in the active state until the timeout expires. Therefore, shorter timeout values are better for saving energy while waiting to transition to sleep. On the other hand, if the timeout value is too long, then there is a good chance that the rest of the idle period is not long enough to amortize the cost of transition to sleep, so the overall cost is actually then higher than it would have been if the device had not gone to sleep at all. A good example of this situation is setting too short of a timeout on a hard drive. Typical hard drives can take up to a few seconds to spin up from sleep. The spinning up process can cost more than twice the power consumption of the active state. Thus, if the timeout value is only a few hundred milliseconds, chances are that a number of times, just as the hard drive spins down, it'll have to immediately spin back up, thus causing large performance and energy overhead. Therefore, the selection of the timeout value has to be done with a good understanding of both device characteristics and the typical workloads. A study of hard drive traces is presented in [43]. One of the major conclusions is that timeout values on the order of multiple seconds are appropriate for many hard drives. This timeout value for hard disks can be shown to be within a factor of two of the optimal policy using competitive ratio analysis [44]. 
Although most commercial implementations have a single fixed timeout value, a number of studies have designed methods for adapting the timeout value to changes in the workloads [45, 46, 47, 48]. Machine learning techniques [46], along with models based on economic analysis [47, 48] have been employed as ways to adapt the timeout value. In another study adaptive policy learns the distribution of idle periods and based on that it selects which sleep state is most appropriate [49]. Competitive analysis is then used to show how close the adaptive policy is to optimum. Adaptive timeout policies also suffer from wasting energy while waiting for the timeout to expire, but hopefully the amount of energy wasted is lower as the timeout value is more closely fine tuned to the changes in the workload.

\subsubsection{Predictive Policies}

Predictive policies attempt to not only predict the length of the next idle period by studying the distribution of request arrivals but also to do so with enough accuracy to be able to transition a device into a sleep state with no idleness. In addition, some predictive policies also wake up a device from the sleep state in anticipation of service request arrival. When the prediction of timing and the length of the idle period is correct, then predictive policies provide a solution with no overhead. On the other hand, if the prediction is wrong, the potential cost can be quite large. If a power manager transitions a device to sleep expecting a long idle period but the idle period is actually short, the overall cost in terms of both energy and performance can be quite large. On the other hand, if the manager predicts an idle period that is short, then it'll wake up a device before it is needed and thus waste energy the same way standard timeout policies do. The quality of idle period prediction is the keystone of these policies. A study on prediction of idleness in hard drives is presented in [50]. Policies based on study of idle period prediction are presented in both $[50,51]$. Two policies are introduced in [51]: the first one is based on a regression model of idle periods, while the second one is based on the analysis of a length of a previous busy period. Exponential averaging is used to predict the idle period length in [52]. Analysis of user interface interactions is used to guide a decision on when to wake up a given component [53]. In multicore designs a signal has been proposed that can be used to notify the system components of an impending request arrival and of instances when no more requests are expected [54]. These signals enable both predictive wakeup and predictive sleep. Both timeout and predictive policies have one thing in common - they are heuristic. None of these policies can guarantee optimality. In contrast, policies that use stochastic models to formulate and solve the power management problem can provide optimal solutions within restrictive assumptions made when developing the system model.

\subsection{Stochastic Policies}

Stochastic policies can loosely be categorized into time- and event-driven and are based on the assumption that all distributions modeling the system are memoryless (e.g., geometric and exponential distributions) or that some distributions are history dependent. Power management policies can be classified into two categories by the manner in which decisions are made: discrete time (or clock based) and event driven. In addition, policies can be stationary (the same policy applies at any point in time) or nonstationary (the policy changes over time). For both discrete time and event-driven approaches, optimality of the algorithm can be guaranteed since the underlying theoretical model is based on Markov chains. An overview of stochastic DPM policies presented to date follows. 
Benini et al. [55] formulated a probabilistic system model using stationary discrete-time Markov decision processes (DTMDP). They rigorously formulate the policy optimization problem and showed that it can be solved exactly and in polynomial time in the size of the system model. The DTMDP approach requires that all state transitions follow stationary geometric distributions, which is not true in many practical cases. Nonstationary user request rates can be treated using an adaptive policy interpolation procedure presented in [56]. A limitation of both stationary and adaptive DTMDP policies is that decision evaluation is repeated periodically, even when the system is idle, thus wasting power. For example, for a $10-\mathrm{W}$ processor, the DTMDP policy with an evaluation period of $1 \mathrm{~s}$ would waste as much as $1800 \mathrm{~J}$ of energy from the battery during a 30-min break. The advantage of the discrete time approach is that decisions are re-evaluated periodically so the decision can be reconsidered, thus adapting better to arrivals that are not truly geometrically distributed.

An alternative to the DTMDP model is a continuous-time Markov decision process (CTMDP) model [57]. In a CTMDP, the power manager (PM) issues commands upon event occurrences instead of at discrete time settings. As a result, more energy can be saved since there is no need to continually re-evaluate the policy in the low power state. Results are guaranteed optimal, assuming that the exponential distribution accurately describes the system behavior. Unfortunately, in many practical cases the transition times may be distributed according to a more general distribution. Figure 4.6 shows a tail distribution of wireless LAN (WLAN) service request interarrival times. The plot highlights that for longer idle times of interest to power management, the exponential distribution shows a very poor fit, while a heavy-tailed distribution, such as Pareto, is a much better fit to the data. As a result, in real implementation the results can be far from optimal [58]. Work presented in [59] uses series and parallel combinations of exponential distributions to approximate general distribution of transition times. Unfortunately, this approach is very complex and does not give a good approximation for the "bursty" behavior observed in real systems $[58,60]$.
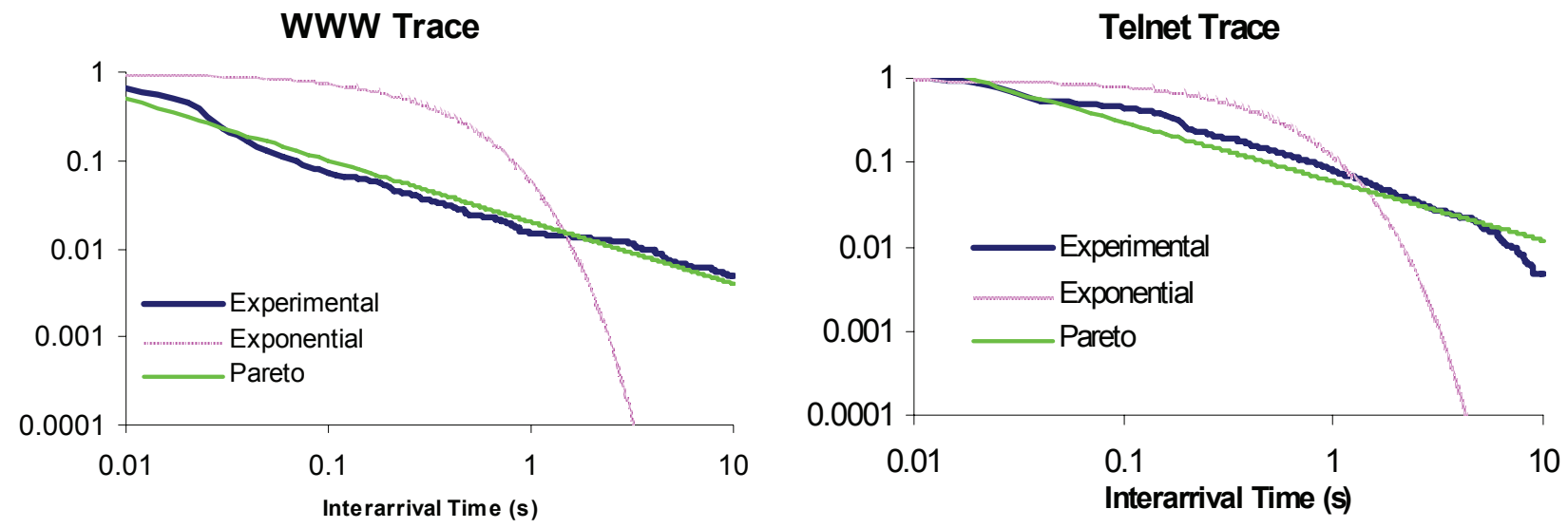

Figure 4.6. WLAN idle-state arrival tail distribution. 
The time-indexed semi-Markov decision process (TISMDP) model has been introduced to solve the DPM optimization problem without the restrictive assumptions of memoryless distribution for system transitions [58]. The power management policy optimization is solved exactly and in polynomial time with guaranteed optimal results. Large savings are measured with the TISMDP model, as it handles general user request interarrival distributions and makes decisions in an event-driven manner. The resulting DPM policy is event driven and can be implemented as a randomized timeout. The value of randomization depends on the parameters of the policy derived from TISMDP optimization. The policy itself is in the form of a distribution that provides the probability of transitioning into a sleep state for every timeout value. Renewal theory has been used in [54] for joint optimization of both DPM and DVS on multicore systemson-a-chip. As in TISMDP, the renewal model allows for modeling a general transition distribution. Probabilistic model checking has been employed to verify the correctness of stochastic policies [61]. Although both TISMDP and renewal models limit the restriction of memoryless distributions only, they do require that all transitions be statistically independent and stationary. An approach presented in [48] removes the stationary assumption by learning the interarrival distribution online. Although this approach does not guarantee optimalization, the quality of estimation has been evaluated using competitive analysis.

\subsection{Operating System and Cross-Layer Dynamic Power Management}

Most power management policies are implemented as a part of an operating system. Intel, Microsoft and Toshiba created an Advanced Configuration and Power Interface specification (ACPI) that can be used to implement power management policies in Windows OS [62]. ACPI gives a structure for implementing DPM but does not actually provide any policies beyond simple timeouts for specific devices. In contrast, NemesisOS and ECOSystem both use pricing mechanisms to efficiently allocate systems' hardware resources based on energy consumption. Both of these approaches use high-level approximations for the energy cost of access to any given hardware resource, thus incurring significant inaccuracies. Their implementation for a different system requires that both kernel- and hardware-specific functions be changed. Another OS-level approach is to compute an equivalent utilization for each hardware component and then to transition a device to sleep when its utilization falls below a predefined threshold [63]. A few energy-aware software implementations integrate information available at multiple system layers to be able to manage energy more efficiently $[64,65]$.

All DPM policies discussed thus far do not perform any traffic reshaping of the incoming workload. Thus their primary goal is to evaluate for a given idle period if a device should transition to sleep. Various buffering mechanisms have been suggested by a number of researchers as a way to enhance the availability of longer idle periods suitable for power management. Buffering for streaming multimedia applications has been proposed as a methodology to lower energy consumption in wireless network interface cards [66]. Similar techniques have been used to help increase the idle times available for spinning down the hard disk power [67]. A general buffer management methodology based on the idea of inventory control has been proposed in [68]. An advantage of this approach is that it can be applied to multiple devices in a single system. When combined with OS-level scheduling, the adaptive workload buffering can be used for both power management and voltage scaling. Shorter idle times created by adaptive buffering can be used to slow down a device, while the longer ones are 
more appropriate for transitions to sleep. In the next section we provide an overview of dynamic voltage scaling methodologies.

\subsection{Dynamic Voltage Scaling}

Two types of voltage scheduling algorithms can be defined for systems with clearly definable task deadlines: intra-task dynamic voltage scaling (DVS) and intertask DVS. Both types of algorithms utilize slack times by slowing down the processor. Intratask DVS algorithms use the available slack time for the task currently running, while intertask DVS provides the extra slack time to the upcoming tasks. The next two sections describe various DVS algorithms that have been presented. A more detailed overview can be found in [69].

\subsection{Intertask Voltage Scaling}

Intertask DVS algorithms (1) run a current task; (2) when the task is completed, calculate the maximum allowable execution time for the next task; (3) assign the supply voltage for the next task; and (4) run the next task. Most intertask DVS algorithms differ during step (2) in computing the maximum allowed time for the next task. A generic intertask DVS algorithm consists of two parts: slack estimation and slack distribution. In distributing slack times, most algorithms use a greedy approach, where all the slack times are given to the next activated task. Slack times generally come from two sources: static slack times are the extra times available for the next task that can be identified statically, while dynamic slack times are caused from runtime variations of task execution. One of the most commonly used static slack estimation methods is to compute the maximum constant speed, which is defined as the lowest possible clock speed that guarantees the feasible schedule of a task set. For example, in earliest deadline first (EDF) scheduling, if the worst-case processor utilization (WCPU) of a given task set is lower than $100 \%$ under the maximum speed, the task set can be scheduled with a new maximum speed that is a fraction of the original speed proportional to the current utilization. The maximum constant speed can be statically calculated as well for rate monotonic (RM) scheduling [70, 71].

Three widely used techniques for estimating dynamic slack times have been proposed: stretching-to-NTA, priority-based slack stealing, and utilization updating. Stretching-to-NTA is based on a slack between the deadline of the current task and the arrival time of the next task (the next task Aarrival time, or NTA). Even though a given task set is scheduled with the maximum constant speed, since the actual execution times of tasks are usually much less than their WCETs, the tasks usually have dynamic slack times. One simple method to estimate the dynamic slack time is to use the arrival time of the next task [70]. The execution of a single task can then be stretched to the NTA. When multiple tasks are activated, there can be several alternatives in stretching options. For example, the dynamic slack time may be given to a single task or distributed equally to all activated tasks.

Priority-based slack stealing allows lower priority tasks to steal the available slack time left from the higher priority tasks. It is also possible for a higher-priority task to utilize the slack times from completed lower-priority tasks. However, the latter type of slack stealing is computationally expensive to implement precisely. Therefore, the existing algorithms are based on heuristics $[72,73]$. The utilization updating techniques estimate the required processor performance at the current scheduling point by recalculating the expected worst-case processor 
utilization using the actual execution times of completed task instances [73]. When the processor utilization is updated, the clock speed can be adjusted accordingly. This method is very simple to implement because only the processor utilization for completed tasks has to be updated at each scheduling point.

\subsection{Intra-Task Voltage Scaling}

Five classes of intra-task DVS algorithms have been proposed that differentiate on where within the program the voltage and clock frequency should be scaled: segment-based, pathbased, memory-aware, stochastic, and hybrid. At a specific program point, two kinds of slack times can be identified: backward slack and forward slack. While the backward slack is generated from the early completion of executed program segments, the forward slack is generated when there is a change to the estimated remaining workload. Segment-based DVS utilizes the backward slack times, while the path-based DVS exploits the forward slack times based on the program's control flow.

Segment-based DVS techniques partition a task into several segments [74, 75]. After executing a task segment, they adjust the clock speed and supply voltage to efficiently exploit the remaining slack time. A key problem of segment-based DVS is how to divide an application into segments. Automatically partitioning an application code is not trivial. One solution is to use both the compiler and the operating system to adapt performance and reduce energy consumption of the processor. Collaborative DVS [76] uses such an approach and provides a systematic methodology to partition a program into segments considering branch, loop, and procedure calls. The compiler annotates the application program with power management hints based on program structure and estimated worst-case performance. The operating system periodically invokes a power management point to change the processor's performance based on the timing information from the power management hints. This collaborative approach has the advantage that the lightweight hints can collect accurate timing information for the operating system without actually changing performance. Further, the periodicity of performance/energy adaptation can be controlled independently of power management hints to better balance the high overhead of adaptation. Programs can also be partitioned based on data types. For example, the required decoding time for each frame in an MPEG decoder can be separated into two parts [76], a frame-dependent (FD) part and a frame-independent (FI) part. Each of these parts then uses the appropriate DVS strategy depending on the available slack times.

Path-based DVS [77] consists of two key steps: (1) predicting the execution path of the application program at compile time and (2) adjusting the clock speed based on the real execution path taken at run time. In the first step, using the predicted execution path, the remaining predicted execution cycles are calculated at a basic code block. For example, worstcase execution path (WCEP) is a metric that can be used. With the predicted value, the initial clock frequency and its corresponding voltage are set assuming that the task execution will follow the predicted execution path. The predicted execution path is called as the reference because the clock speed is determined based on the execution path. At run time, if the actual execution deviates from the (predicted) reference path (say, by a branch instruction), the clock speed can be adjusted depending on the difference between the remaining execution cycles of the reference path and those of the newly deviated execution path. If the new execution path takes significantly longer to complete its execution than the reference execution path, the clock speed 
should be increased to meet the deadline constraint. On the other hand, if the new execution path can finish its execution earlier than the reference execution path, the clock speed can be lowered to save energy. For run-time clock speed adjustment, voltage scaling code is inserted into the selected program locations at compile time. The branching edges of the control flow graph, i.e., branch or loop statements, are the candidate locations for inserting voltage scaling calls. Although WCEP-based DVS reduces the energy consumption significantly while guaranteeing the deadline, this is a pessimistic approach because it always predicts that the longest path will be executed. If the average-case execution path (ACEP) is used as a reference path, a more efficient voltage schedule can be generated. (The ACEP is an execution path that will be most likely to be executed.) To find the average-case execution path, the profile information on the program execution should be used.

Memory-aware DVS differs from path-based DVS in the type of CPU slacks being exploited. While path-based DVS takes advantage of the difference between the predicted execution path and the real execution path of applications, the memory-aware class exploits slacks from the memory stalls. Once the program regions in which the CPU is mostly idle because of memory stalls are identified, then they can be slowed down for energy reduction. If the system architecture supports the overlapped execution of the CPU and memory operations, such a CPU slowdown will not result in serious system performance degradation, as the slow CPU speed would be hidden behind the memory hierarchy accesses that are on the critical path. There are two kinds of approaches to identify the memory-bound regions: analyzing a program at compile time and monitoring run-time hardware events. The compiler-directed approach [78] partitions a program into multiple regions. It assigns a different slowdown factor to each region in order to maximize the overall energy savings without violating the global performance penalty constraint. The granularity of the region needs to be large enough to compensate for the overhead of voltage and frequency adjustments. Event-driven DVS [79, 80] makes use of run-time information about the external memory access statistics. The technique relies on dynamically constructed regression models that allow the CPU to calculate the expected workload and slack time for the next time slot. This calculation is achieved by estimating and exploiting the ratio of the total off-chip access time to the total on-chip computation time.

Stochastic DVS estimates a program's execution time using stochastic techniques [81, 82]. It finds a speed schedule that minimizes the expected energy consumption while still meeting the deadline. A task starts executing at a low speed and then gradually accelerates its speed to meet the deadline. Finally, hybrid DVS overcomes the main limitation of intra-DVS techniques. Intratask techniques have no global view of the task set in multitask environments. Hybrid techniques therefore select between intra- and inter-DVS modes [83]. In the inter- mode, the slack time identified during the execution of a task is transferred to the following tasks. Therefore, the speed of the current task is not changed by the slack time produced by it. In the intra- mode, the slack time is used for the current task by reducing its own execution speed. 


\subsection{Conclusion}

Power consumption is one of the key design and optimization parameters in today's embedded sensing systems. Two approaches that enable systems to save power by adapting to changes in environment have been proposed: dynamic power management and dynamic voltage scaling. DVS techniques take advantage of workload variations within a single task execution as well as workload fluctuations from running multiple tasks, and adjusts the supply voltage, thus reducing the energy consumption of the processing part in the embedded system. DPM techniques identify idle system components using various techniques and place the identified components into low-power states. DPM algorithms can be applied to processing, communication, and other parts of the embedded system design. They typically provide larger power savings, but at a larger performance penalty. Since both approaches are based on the system idleness, DVS and DPM can be combined into a single power management framework. For example, shorter idle periods are more amiable to DVS, while longer ones are more appropriate for DPM. Thus, a combination of the two approaches is necessary for more powerefficient embedded systems.

\section{ENERGY HARVESTING METHODS AND APPLICATIONS FOR SHM}

The process of extracting energy from the environment or from a surrounding system and converting it to useable electrical energy is known as energy harvesting. Recently, there has been a surge of research in the area of energy harvesting. This increase in research has been brought on by modern advances in wireless technology and low-power electronics such as MEMS devices. Given the wireless nature of some emerging sensors, it becomes necessary that they contain their own power supply, which is, in most cases, conventional batteries. However, when the battery has consumed all of its power, the sensor must be retrieved and the battery replaced. Because of the remote placement of these devices, obtaining the sensor simply to replace the battery can become a very expensive and tedious, or even impossible, task. For instance, in civil infrastructure SHM applications, it is often desirable to embed the sensor units into the structures, making battery replacement unfeasible. If ambient energy in the surrounding medium can be obtained and utilized, this captured energy can then be used to prolong the life of the power supply or, ideally, provide unlimited energy for the lifespan of the electronic device. Given these reasons, the amount of research devoted to energy harvesting has been rapidly increasing, and the SHM and sensing network community have investigated energy harvesters as an alternative power source for the next generation of embedded sensing systems. The sensors and electronics powered by energy harvesters can be placed in any inaccessible location, for instance, on bridges, ships, or aerospace structures, to provide vital information on structural, environmental, and operational conditions.

The sources of typical ambient energies are sunlight, thermal gradient, human motion and body heat, vibration, and ambient RF energy. Several excellent articles reviewing possible energy sources for energy harvesting can be found in the literature [32, 84, 85, 86, 87, 88, 89]. Fry et al. [84] provides an overview of portable electric power sources that meet US military special operation requirements. The potential power sources surveyed by the report are thermoelectric generators, mechanical vibration devices such as piezoelectric devices and wind turbines, solar cells, and exotic portable power sources including tapping the laser beam and ambient electromagnetic radiation, as well as traditionally available portable power sources such as batteries and fuel cells. Although the report is concerned only with portable energy sources for 
military combat missions, it provides critical insight into future research trends in energy harvesting. For instance, the report defines a list of general attributes intended to suggest what a standard characterization of different portable energy supplies should include. The list includes electrical (energy density, total energy content, power density, maximum voltage and current, RF emission power, electrical interconnects), physical (size/shape, weight), environmental (acoustic emission power, mechanical shock tolerance, electrical shock tolerance, water resistance, operating temperature range), operational (energy requirements for recharging, orientation), maintenance (testing requirements), safety, and disposal. The report also categorizes the energy harvesters by their technical maturity, indicating those that still require considerable development before their feasibility for use in the field can be demonstrated. Piezoelectric devices that have been extensively investigated by many researchers in the early 2000s were set to this category. The report concludes with the suggestion that the development of a general standard to address the technical capabilities of energy sources should be pursued in such a way that system designers and integrators are able to assemble components to produce a satisfactory final design. It is still questionable if such design standards currently exist after the tremendous research efforts dedicated to energy harvesting.

Roundy [86] compares the energy density of available and portable energy sources, shown in Table 5.1. He concludes that for a device whose desired lifetime is in the range of 1 year or less, battery technology alone is sufficient to provide enough energy. However, if a device requires a longer service life, which is often the case, an energy harvester can provide a better solution than battery technologies. Paradiso and Starner [89] point out that battery technology has evolved very slowly in mobile computing: battery energy density has increased only by a factor of 3 since 1990. Over the same time period, disk storage density has increased by a factor of 1300 and CPU speed by a factor of nearly 800. They also provide the energy harvesting capabilities of different sources (shown in Table 5.2), which are slightly different from those suggested by Roundy [86]. Glynne-Jones and White [85], Qiwai et al. [87], and Mateu and Moll [88] also summarize the basic principles and components of energy harvesting techniques, including piezoelectric, electrostatic, magnetic induction, and thermal energy. A common suggestion listed in these articles is the combined use of several energy harvesting strategies in the same device so that the harvesting capabilities in many different situations and applications can be increased. Furthermore, energy consumption can be minimized in an effort to close the gap between required and harvested energy [88].

This section provides an up-to-date assessment of available energy harvesting methods suitable for potential SHM sensing applications. This section is not intended to provide an exhaustive literature review, as this area is very broad and useful review articles are already available in the literature. Instead, this section will provide a concise introductory survey on the topic and outline the current status of energy harvesting as applied to relevant themes in SHM.

\subsection{Converting Mechanical Vibration to Electrical Energy}

One of the most effective methods of implementing an energy harvesting system is to use mechanical vibration to apply strain energy to a piezoelectric material or to displace an electromagnetic coil. Energy generation from mechanical vibration usually uses ambient vibration around the energy harvesting device as an energy source and then converts it into useful electrical energy. The research in this area has made use of mechanical vibration in order to quantify the efficiency and amount of energy capable of being generated and converted, as well as the amount needed to power various electronic systems. 
Table 5.1. Comparison of energy sources. (Source: Roundy [86])

\begin{tabular}{|c|c|c|c|c|}
\hline \multirow{8}{*}{ 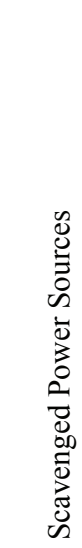 } & & $\begin{array}{c}\text { Power Density } \\
\left(\mu W / \mathrm{cm}^{3}\right) \\
1 \text { Year Lifetime }\end{array}$ & $\begin{array}{c}\text { Power Density } \\
\left(\mu \mathrm{W} / \mathrm{cm}^{3}\right) \\
10 \text { Year Lifetime }\end{array}$ & Source of Information \\
\hline & Solar (Outdoors) & $15,000-$ direct sun & 15,000 - direct sun & Commonly Available \\
\hline & Solar (Indoors) & 6 - office desk & 6 - office desk & Roundy [86] \\
\hline & Vibrations & 200 & 200 & Roundy et al. [99] \\
\hline & Acoustic Noise & $0.003 @ 75 \mathrm{~dB}$ & $0.003 @ 75 \mathrm{~dB}$ & Theory \\
\hline & & $0.96 @ 100 \mathrm{~dB}$ & $0.96 @ 100 \mathrm{~dB}$ & \\
\hline & Daily Temp. Variation & 10 & 10 & Theory \\
\hline & Temperature Gradient & $15 @ 10^{\circ} \mathrm{C}$ gradient & $15 @ 10^{\circ} \mathrm{C}$ gradient & Stordeur and Stark 1997 [90] \\
\hline \multirow{5}{*}{ 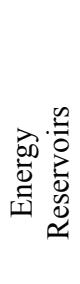 } & Shoe Inserts & 330 & 330 & Starner 1996 [95] \\
\hline & Batteries (nonrecharge Lithium) & 45 & 3.5 & Commonly Available \\
\hline & Batteries (rechargeable Lithium) & 7 & 0 & Commonly Available \\
\hline & Fuel Cells (methanol) & 280 & 28 & Commonly Available \\
\hline & Nuclear Isotopes (Uranium) & $6 \times 10^{6}$ & $6 \times 10^{5}$ & Commonly Available \\
\hline
\end{tabular}

Table 5.2. Energy harvesting demonstrated capabilities. (Source: Paradiso and Starner [89])

\begin{tabular}{|c|c|}
\hline Energy Source & Performance \\
\hline Ambient radio frequency & $<1 \mu \mathrm{W} / \mathrm{cm}^{2}$ \\
Ambient light & $100 \mathrm{~mW} / \mathrm{cm}^{2}($ directed toward bright sun) \\
& $100 \mu \mathrm{W} / \mathrm{cm}^{2}$ (illuminated office) \\
Thermoelectric & $60 \mu \mathrm{W} / \mathrm{cm}^{2}$ \\
Vibrational microgenerators & $4 \mu \mathrm{W} / \mathrm{cm}^{3}($ human motion $-\mathrm{Hz})$ \\
& $800 \mu \mathrm{W} / \mathrm{cm}^{3}($ machines $-\mathrm{kHz})$ \\
$1 \mathrm{~mW} / \mathrm{cm}^{2}$ \\
Ambient airflow \\
Push buttons & $50 \mu \mathrm{J} / \mathrm{N}$ \\
Hand generators & $30 \mathrm{~W} / \mathrm{kg}$ \\
Heel strike & $7 \mathrm{~W}$ potentially available $(1 \mathrm{~cm} \mathrm{deflection} \mathrm{at} 70 \mathrm{~kg}$ per $1 \mathrm{~Hz}$ walk $)$ \\
\hline
\end{tabular}

The concept of utilizing piezoelectric material for energy generation has been studied by many researchers over the past few decades. Piezoelectric materials form transducers that are able to interchange electrical energy and mechanical motion or force. These materials, therefore, can be used as mechanisms to transfer ambient vibration into electrical energy that may be stored and used to power other devices. A full description of the piezoelectric effect and the methods used to model these materials' behavior is beyond the scope of this report. However, a significant number of journal papers and conference proceedings develop accurate models and discuss the fundamentals of these materials in great detail [20, 91, 92]. Furthermore, an overview of the application of piezoelectric transducers as energy harvesters has been recently given by Sodano et al. [32] and duToit et al. [93]. 
One early study into energy harvesting by Hausler et al. [94] investigated the ability to generate energy from the expansion and contraction of the rib cage during breathing. A prototype of the energy harvesting system was constructed using polyvinylidene fluoride (PVDF) film and was implemented in vivo on a mongrel dog. The prototype was demonstrated to produce a peak voltage of $18 \mathrm{~V}$, which corresponded to a power of about $17 \mu \mathrm{W}$. The work of Starner [95] brought the possibility of energy harvesting locations around the human body to the attention of many researchers. The paper contained a survey of various power generation methods ranging from body heat and breath to finger and upper limb motion. An analysis of the power available from each of the different sources was presented. He calculates that approximately $67 \mathrm{~W}$ of power is lost during walking and that a piezoelectric device mounted inside a shoe with a conversion efficiency of $12.5 \%$ could achieve $8.4 \mathrm{~W}$ of power. Kymissis et al. [96] developed a piezoelectric system that would harvest the energy lost during walking and used it to power a radio transmitter, shown in Figure 5.1. The devices that were considered included a Thunder actuator constructed of piezoceramic composite material located in the heel and a multilayer PVDF foil laminate patch located in the sole of the shoe. The peak powers were observed to approach $20 \mathrm{~mW}$ for the PVDF stave and $80 \mathrm{~mW}$ for the piezoelectric actuator. However, due to slow excitation, the average power generated from both the PVDF and the Thunder actuator was significantly lower, approximately $1 \mathrm{~mW}$ and $2 \mathrm{~mW}$, respectively, shown in the figure. It was found that the two piezoelectric devices used produced sufficient energy to power a transmitter that could send a 12-bit RFID code every 3-6 steps. Another investigation into the use of piezoelectric materials for power harvesting from the motion of humans and animals was performed by Ramsey and Clark [97], who studied the ability to power an in vivo MEMS application. The research used a thin square plate driven by blood pressure to provide energy and was shown to be capable of powering the electronics if they were used intermittently. Although these wearable energy harvesters are not suitable for powering SHM sensor nodes, which should rely on ambient vibration of structures, these works demonstrated the potential of piezoelectric energy harvesting devices as an alternative energy source for self-powered electronics. Further, the feasibility of using the harvested energy for wirelessly transmitting data was demonstrated and subsequently gained the attention of many researchers in the area of self-powered wireless sensors.
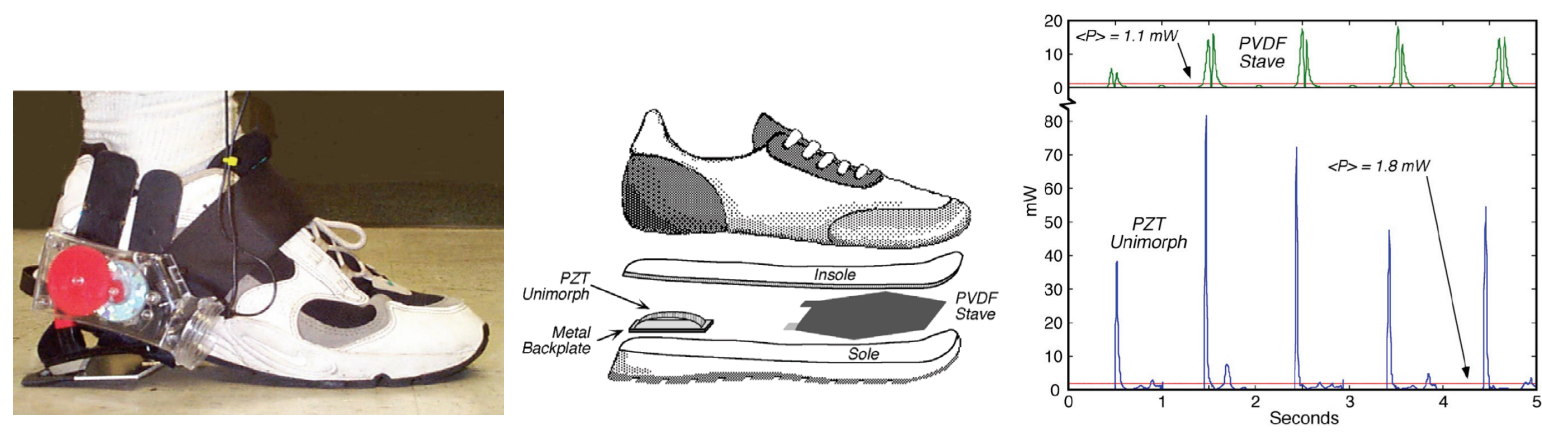

Figure 5.1. Schematic and results of energy harvesting shoe. (Source: Kymissis et al. [96]) 
Umeda et al. [98] quantified the amount of energy that could be produced when a steel ball impacted a piezoelectric plate. The authors used an equivalent circuit model to predict the energy while modifying numerous parameters in the system to find the best combination of parameters for energy harvesting. It was determined that the efficiency increased if the mechanical quality factor (the reciprocal of internal friction) of the piezoelectric materials increased, the electromechanical coupling coefficient increased, and the dielectric loss decreased. Roundy et al. [99] studied low-level vibrations as a power source for wireless sensor nodes. In this study, two piezoelectric patches were attached to a steel cantilevered beam and used to collect mechanical energy from vibrations. By using an excitation force designed to simulate the vibration of an operating microwave oven, the piezoelectric material achieved a $70 \mu \mathrm{W} / \mathrm{cm}^{3}$ power density.

Another example of energy harvesting is the development of an electrical power generating system that extracts energy from the flow of moving water in pipes, streams or currents [100]. The generating systems are eel-like structures made from PVDF films. These "eels" are scalable in size and have the capacity to generate milliwatts to many watts depending on the system size and the water flow velocity. An illustration of the eel concept developed by Ocean Power Technologies, Inc. is shown in Figure 5.2.

Sodano et al. [101] estimated the power output from a piezoelectric cantilever auxiliary structure attached to an automobile compressor. A $40-\times 62-\mathrm{mm}$ piezoelectric patch mounted to a fixed-free, $40-\times 80-\mathrm{mm}$ plate was able to charge a $40-\mathrm{mAh}$ button cell battery in one hour. Sodano et al. [102] also formulated a model of a power harvesting system that consisted of a cantilever beam with piezoelectric patches attached. The model was verified on a cantilever beam experiencing a base excitation from the clamped condition. The model was found to accurately estimate the energy generated and was also used to demonstrate the damping effect of a piezoelectric energy harvester. The development of an accurate analytical model to estimate the power output from the piezoelectric transducers and to understand the effects of several components, including mechanical and electrical loads and electrical circuits, has received considerable attention by energy harvesting researchers. As such, various efforts on analytical modeling and analysis of piezoelectric energy harvesting can be found in the literature [93, 103, $104,105,106,107]$.

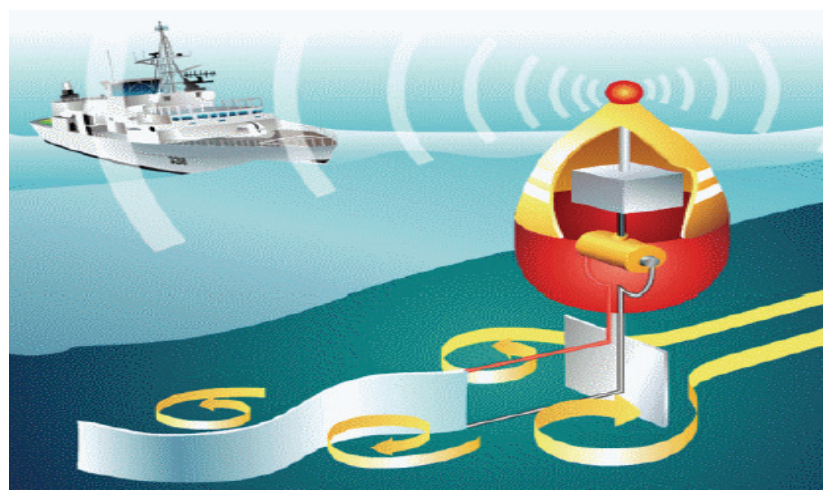

Figure 5.2. Energy harvesting "eel" concept. (Source: Ocean Power Technologies, Inc.) 
The efficiency of the piezoelectric material in a stack configuration for the purpose of electric energy generation was analyzed by Goldfarb and Jones [108]. An analytical model is presented and the fundamental problem with generating electrical power from the piezoelectric material is that it stores the majority of the energy produced and returns it to the excitation source that initially caused the charge to be generated. Therefore, it is suggested that the maximum efficiency of power generation can be achieved by minimizing the amount of energy stored inside the piezoelectric material. It was found that at frequencies above $100 \mathrm{~Hz}$, the efficiency of the stack actuator was negligible and that the highest efficiency was obtained at a $5 \mathrm{~Hz}$, which is much lower than the first mechanical and electromechanical resonances of the stack. Although the piezoelectric stack configuration utilizes the higher electromechanical coupling mode $\left(\mathrm{d}_{33}\right)$ compared to that of the patch configuration $\left(\mathrm{d}_{31}\right)$, the patch configuration holds great advantages in energy conversion because the excitation is more easily achieved by environmental sources [88, 97]. Accordingly, a cantilever beam with the piezoelectric patches attached in either a unimorph or a bimorph form is the most common configuration for energy harvesting. Others utilized the shape of membranes under pressure loading [106, 109] and plates with a Helmholtz resonator under fluid/acoustic loading [110]. Recently, the development of MEMS-scale micro power generator has received considerable attention, as piezoelectric materials are suitable for microfabrication $[93,111,112$, 113]. For instance, the microscale piezoelectric harvester developed by Jeon et al. [111], shown in Figure 5.3, generated a maximum DC voltage of $3 \mathrm{~V}$ and a maximum continuous electrical power of $1 \mu \mathrm{W}$ under the first resonance frequency of $13.9 \mathrm{kHz}$.

Other than traditional piezoceramic and PVDF materials, several researchers have investigated the energy harvesting performance of lead magnesium niobate-lead titanate (PMN-PT) single-crystal devices [103, 114], a macro-fiber composite actuator [101], and a "cymbal" piezoelectric transducer [115], which all exhibit much higher electromechanical coupling properties than traditional transducers and hence show much better performance. For instance, a cymbal transducer with a $29-\mathrm{mm}$ diameter and $1-\mathrm{mm}$ thickness produced $39 \mathrm{~mW}$ power at a frequency of $100 \mathrm{~Hz}$ under a force of $7.8 \mathrm{~N}$, which is much higher than values reported in the literature from traditional piezoelectric materials.

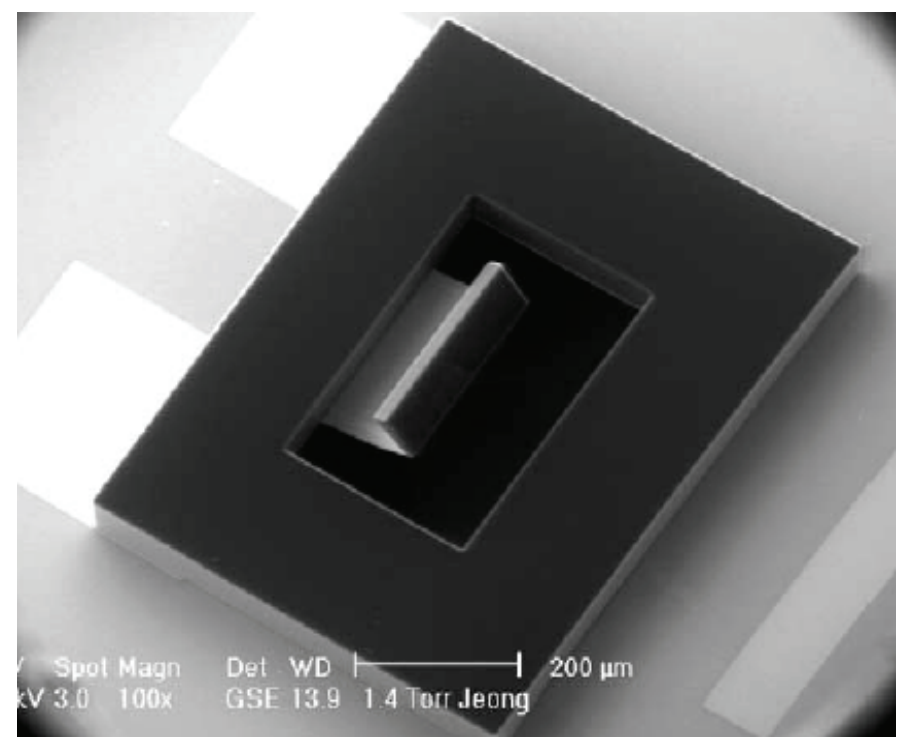

Figure 5.3. The fabricated microscale piezoelectric generator. (Source: Jeon et al. [111], reprinted with permission from Elsevier.) 
To achieve higher efficiency, it is necessary to match the resonance frequency of the transducer with the most distinct frequency of the vibration source. Cornwell et al. [116] adjusted various mechanical parameters, including the resonant frequency and the location of a harvester, to maximize the strain induced in the piezoelectric element in order to improve power output. The power generation was increased by a factor of 25 when the frequency of the harvesting device was well tuned to that of the structure. Roundy and Wright [105] also suggested that the harvesting system should be designed such that the harvester could be excited at its resonance. The proof mass was used to maximize the power output, shown in Figure 5.4. The vibration present in a structure is, however, usually much lower than the resonance of a harvesting device and often changes during operation; therefore, this vibration does not always effectively couple energy to the harvester. The optimization of the transducer setup and geometry is one of the most challenging tasks during the design, but it has received less attention from researchers.

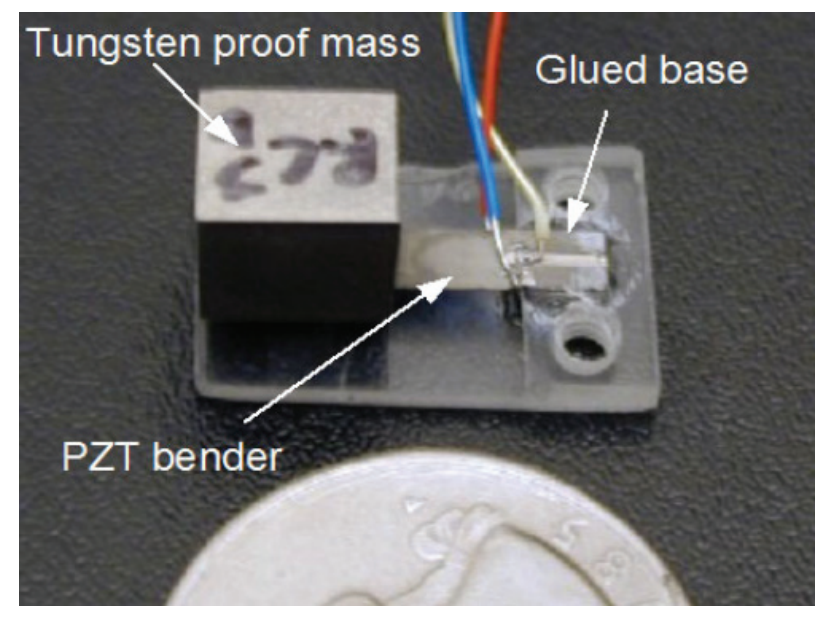

Figure 5.4. A piezoelectric generator with a proof mass. (Source: Roundy and Wright [105])

With respect to ambient vibration, there is another possible way of converting mechanical energy into electricity. The electromagnetic systems are composed of a coil and a permanent magnet attached to a spring. The mechanical movement of the magnet, which is caused by structural vibration, induces a voltage at the coil terminal and this energy can be delivered to an electrical load. The amount of power produced is maximum at resonance of a device and proportional to the square of the peak mass displacement. Furthermore, a large proof mass (a magnet) with large coil areas will perform better than smaller ones, although the size and displacement will be limited by the spring and the housing of the device. Williams and Yates [117] proposed a device that generates electricity using an electromagnetic transducer. A harmonic analysis of the generator was performed and it was determined that the amount of power generated was proportional to the cube of the vibration frequency, which illustrated that the generator was likely to perform poorly at low frequencies. It was also determined that a low damping factor within the electromagnetic system was required to maximize power generation, therefore, the design must allow for large deflections of the mass. For a typical device the predicted power generation was $1 \mu \mathrm{W}$ at an excitation frequency of $70 \mathrm{~Hz}$ and $0.1 \mathrm{~mW}$ at $330 \mathrm{~Hz}$, with a mechanical deflection of $50 \mu \mathrm{m}$. Yuen et al. [118] developed an electromagnetic-based micro energy converter that can be packaged into an AA battery-size container. The converter is able to charge a capacitor to a $1.6 \mathrm{~V} \mathrm{DC}$ level in less than $1 \mathrm{~min}$. The device was used to serve as a power 
supply for a wireless temperature sensing system. Glynne-Jones [119] designed a miniature electromagnetic power generator, shown in Figure 5.5, which is based around four magnets coupled to a cantilevered coil. The device, with a volume of $3.15 \mathrm{~cm}^{3}$, could produce a peak power of $3.9 \mathrm{~mW}$ with an average power of $157 \mu \mathrm{W}$ when mounted on the engine block of the car. Mizuno and Chetwynd [120] also investigated an electromagnetic micro generator with a predicted power output of $6 \mathrm{nW}$ for a typical single-element generator with a very low voltage. The authors suggested deploying a "stacked" array configuration to increase the output. Recently Stephen [121] has analyzed the dynamics of an electromagnetic energy generator in detail. He concluded that the maximum power is delivered when the resistance of an electrical load is equal to the sum of the coil's internal resistance and the electrical analogue of the mechanical damping coefficient. There were conflicting claims in the past, some suggesting that the impedance of the electrical load should be matched to the source impedance of the coil, while others suggest that power delivery is optimized when mechanical and electrical damping ratios are equal.

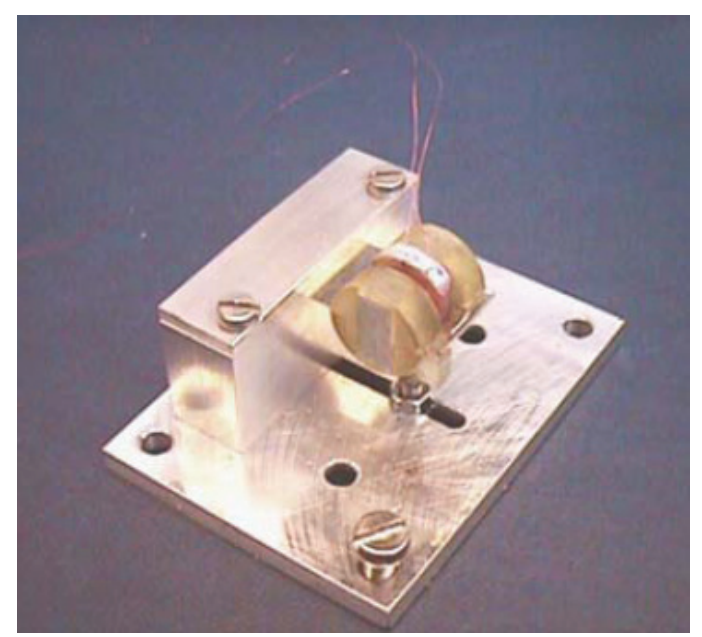

Figure 5.5. An electromagnetic generator.

(Source: Glynne-Jones et al. [119], reprinted with permission from Elsevier.)

Poulin et al. [122] presented a comparative study of electromagnetic and piezoelectric energy conversion systems. These authors found that the two systems are in complete duality in every respect (some elements shown in Table 5.3) after a quantitative study using realistic values of geometrical and physical parameters. The authors suggested that the piezoelectric system is well suited to energy generation for microsystems because of a higher power density, and they recommended electromagnetic systems for medium-scale applications. Roundy [123] pointed out that although there have been many publications in energy harvesting, a solid basis for comparison between basic technologies has not been well documented. He described several basic theories in energy harvesting, including electromagnetic, piezoelectric, and magnetostrictive harvesters. Magnetostrictive materials exhibit the coupling property between magnetic and mechanical domains. Only a few studies explored the energy harvesting potential of magnetostrictive materials [124]. Roundy concluded that, in addition to the input vibration, the power output depends on the system coupling coefficient, the quality factor of the device, the mass density of the generator, and the degree to which the electrical load maximizes the power transmission. He also concluded that the voltage output of electromagnetic devices scales down as size decreases, while for piezoelectric transducers, current, not voltage, will scale down because of the capacitive nature 
of the devices. duToit et al. [93] summarized some experimental and analytical results on vibration-based energy harvesting devices published in the literature. The device sizes vary from micro $\left(0.01 \mathrm{~cm}^{3}\right)$ to macro scale $\left(75 \mathrm{~cm}^{3}\right)$, and the energy generated ranges from $1 \mu \mathrm{W}$ to a couple of $\mathrm{mW}$. However, they conclude that it is a somewhat daunting task to compare the performance of those energy harvesting systems because they use different energy conversion schemes, which vary in size and mass; they have different input frequency spectra; and the various systems and structures that harvesters were installed in. These parameters are not always clearly documented in the published papers. The authors suggested that the power density $\left(\mathrm{W} / \mathrm{cm}^{3}\right.$ or $\mathrm{W} / \mathrm{kg}$ ) or the efficiency parameter would be good indicators for comparing the performance of each device.

Table 5.3. Comparison elements between electromagnetic and piezoelectric systems. (Source: Poulin et al. [122], reprinted with permission from Elsevier.)

\begin{tabular}{|c|c|c|}
\hline System & Electromagnetic & Piezoelectric \\
\hline Constraint & Low & High \\
Displacement & High & Low \\
Voltage & Adjustable & High \\
Current & Adjustable & Low \\
Resonant Frequency & Adjustable & High \\
Output impedance & Resistive & Capacitive \\
Adapted Load & Adjustable & High \\
\hline
\end{tabular}

Because vibration-based energy harvesters are still in the development stage, only a few commercial solutions are available. Most research efforts are still in proof-of-concept demonstrations in a laboratory setting. Microstrain, Inc. [125] developed a prototype of piezoelectric-based energy harvester, shown in Figure 5.6. The sensor node is equipped with temperature and humidity sensors with wireless telemetry. It is claimed that the piezoelectric harvester can produce up to $2.7 \mathrm{~mW}$ of instant power at $57 \mathrm{~Hz}$ vibration. Perpetuum, Inc. [126] commercialized electromagnetic energy converters, which are capable of generating up to $3.3 \mathrm{~V}$ and $5 \mathrm{~mW}$ of instant power under the $100 \mathrm{mg}$ vibration. The frequency could be tuned to in the range of 47-100 Hz. Ferro Solution, Inc. [127] also produced electromagnetic generators that have a $9.3 \mathrm{~mW}$ power capability with $100 \mathrm{mg}$ input vibration.

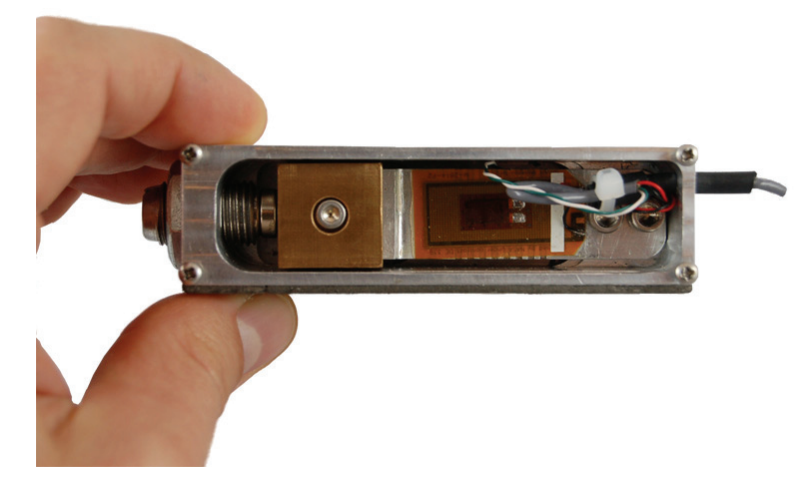

Figure 5.6. A prototype of sensor node with piezoelectric energy harvester. (Source: Image courtesy Microstrain, Inc., [125], reprinted with permission.) 


\subsection{Converting Thermal Energy to Electrical Energy}

A second method of obtaining energy from ambient sources is through the use of thermoelectric generators that capitalize on thermal gradients. Thermoelectric generators (TEGs) use the Seebeck effect, shown in Figure 5.7, which describes the current generated when the junction of two dissimilar metals experiences a temperature difference. Using this principle, numerous p-type and n-type junctions are arranged electrically in series and thermally in parallel to construct the TEG. Thus, when an electrical current is applied to the TEG a thermal gradient is generated, allowing the device to function as a small solid state heat pump. Inversely, if a thermal gradient is applied to the device, it will generate an electrical current that can be utilized to power other electronics.

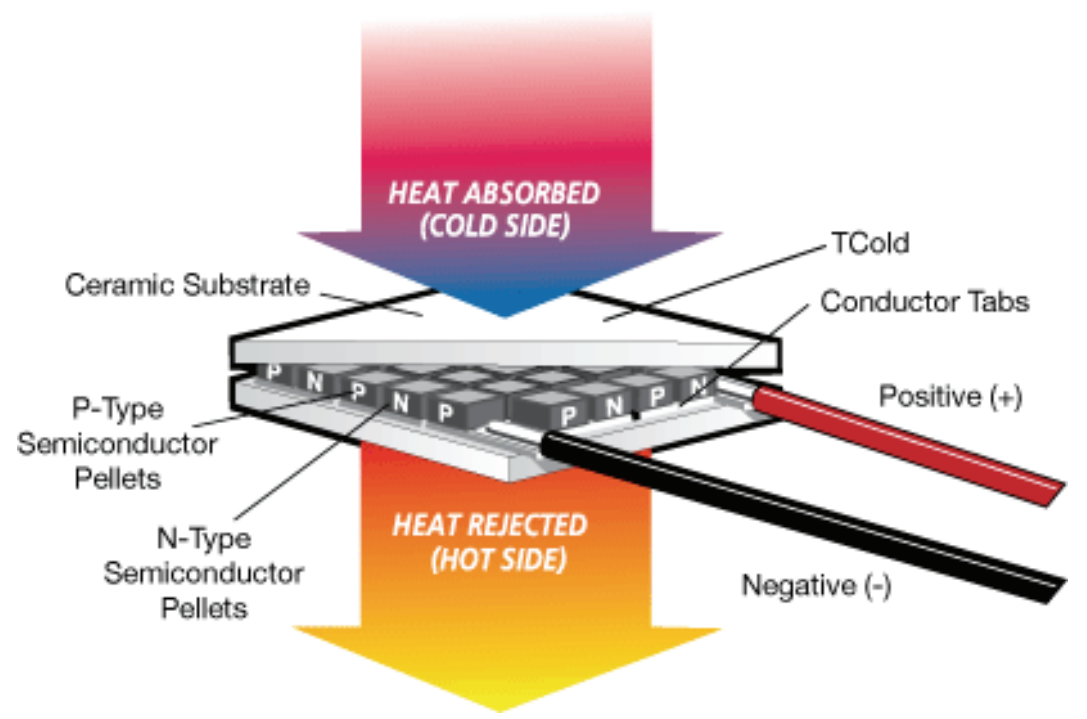

Figure 5.7. Schematic of the Seebeck effect. (Source: www.tellurex.com)

Thermoelectric generators have been used for capturing ambient energy in various applications. Lawrence and Snyder [128] suggest a potential method of retrieving electrical energy from the temperature difference that exists between the soil and the air. To test their concept, a prototype was built without the thermoelectric generator and the heat flow was measured to estimate the amount of power that could be obtained. The results showed that a maximum instantaneous power of approximately $0.4 \mathrm{~mW}$ could be generated by the thermoelectric device. Rowe et al. [129] investigate the ability to construct a large thermoelectric generator capable of supplying 100 watts of power from hot waste water. The system tested used numerous thermoelectric devices placed between two cambers, one with flowing hot water and the other with cold water flowing in the opposite direction, thus maximizing the heat exchange. With a total of 36 modules, each with 31 thermocouples, 95 watts of power could be generated. Fleming et al. [130] investigated the use of TEG for powering microscale air vehicles. A TEG was mounted on the exhaust system of an internal combustion engine that was shown to generate $380 \mathrm{~mW}$ of power. Several authors have studied the use of thermoelectric generators for obtaining waste energy from the exhaust of automobiles. Birkholz et al. [131]worked with Porsche to develop a TEG unit that would fit around the exhaust pipe. The unit was experimentally tested and found to generate an open circuit voltage of 22 volts and a total power of $58 \mathrm{~W}$. Similarly, Matsubara [132] constructed an exhaust system using ten TEG modules and a liquid heat exchanger to maximize 
the thermal gradient. The system was tested on a $2000 \mathrm{cc}$ class automobile and shown to produce $266 \mathrm{~W}$ of power. Bass et al. [133] investigated the placement of a thermoelectric generator in the vertical muffler of a class 8 diesel truck. The system generated $1 \mathrm{~kW}$ of power, thus allowing it to be employed as a substitute for the truck's alternator. By removing the alternator from the engine, the power delivered to the driveshaft was increased by three to five horsepower, providing an increase in fuel efficiency and a reduction in emissions. TEGs are now commonly used in hybrid vehicles to recharge supercapacitors/batteries. For more information on TEG applications in automobiles, see Vázquez et al. [134].

As described, the idea to use thermoelectric devices to capture ambient energy from a system is not a new concept. However, in many cases, the research efforts utilize liquid heat exchangers or forced convection that significantly improves heat flow and power generation but requires complex cooling loops and systems. Therefore, Sodano et al. [135] investigated the use of TEGs as energy harvesting devices that do not have an active heat exchanger but function as a completely passive energy scavenging system. Two potential applications are investigated, utilizing solar radiation and harvesting of waste heat. For each application, an experimental prototype was constructed and tested to determine the effectiveness in recharging a discharged nickel metal hydride battery. The results showed that the thermoelectric generator does produce significantly more power than a piezoelectric device and that the charge time needed to recharge a battery is significantly lower.

The TEG is a mature technology and a reliable energy converter with no moving parts compared to vibration-based harvesters. The TEG has been actively studied for the last three decades and the literature in this area is extensive. One of the drawbacks of this technology is low efficiency $(<5 \%)$ if there is a small temperature gradient present. Further, the fabrication cost is high, and the volume and weight are still too large for microscale sensing systems. Therefore, with the recent advances made in nanotechnologies, the fabrication of MEMS-scale TEG devices have been actively studied [136, 137, 138]. For instance, $0.5 \mathrm{~cm}^{2}$ of a new thermoelectric thin film developed by Applied Digital Solutions produces $1.5 \mu \mathrm{W}$ of power with only a $5^{\circ} \mathrm{C}$ temperature gradient [139].

It is worthwhile to note that TEGs have long been used in NASA space vehicles, such as the Voyager and Cassini probes, as sunlight is not always available for vehicles traveling to the outer reaches of the solar system and beyond. These vehicles use heat generated by the decay of radioactive materials (e.g., plutonium-238) to produce electricity using the TEG. These systems can generate high power in the few watts to kilowatts range for over a decade. However, these devices are not useful in low-power applications because as the radioactive material's size decreases, the surface-to-volume ratio of the heat source increases, which imposes difficulties in maintaining the necessary temperature gradient and efficiency for TEGs. For microscale applications using radioactive material sources, such as tritium, two different harvesting approaches have been proposed. One approach is using the beta-voltaic effect, which works much like a solar cell [140]. Although the beta-voltaic effect suffers from low efficiencies, Sun et al. [141] improved the efficiency by a factor of 10 with a new wafer design that provides more reactive surface to interact with the decay particles, and hence harnesses the energy from the tritium more effectively. Their commercial product, called Betabatt, can provide an energy density of $125 \mu \mathrm{W} / \mathrm{cm}^{3}$ for 12-20 years [142]. Another approach commonly employed with microscale radioactive power sources to harness energy is to combine the radioactive materials with a piezoelectric harvester. Such systems are referred to as a radioisotope-powered piezoelectric generator $[143,144]$. The principle behind this technique is to capture the kinetic 
energy of particles emitted by radioactive materials to actuate a piezoelectric cantilever beam that produces electricity in the range of tens of $\mu \mathrm{W}$. With the overall conversion efficiency of $4 \%$, the device, called a "nuclear micro-battery," with $10 \mathrm{mg}$ of polonium-210 contained in a $1-\mathrm{cm}^{3}$ housing can produce $50 \mathrm{~mW}$ of electric power for four months. An ongoing effort is an attempt to boost the efficiency of this device to $20 \%$.

The use of radioactive materials for energy harvesting provides certain advantages over traditional chemical batteries. Radioactive materials have much higher energy densities and last as long as it takes for the isotope and its daughter isotopes to decay to a stable state, albeit with diminishing energy levels over this time. Many chemical batteries, however, have limited shelflives of three to four years because of self-discharging. Other issues that must be considered when using radioactive materials as an energy source are the types of decay products that are produced, the half-lives of the materials, and the ability of the TEG to accommodate a timevarying energy flux. Ideally, the isotope should be primarily an alpha or beta emitter, otherwise there may be a need to harden the electronics for gamma or neutron decay products. Also, the isotope's decay chain must be studied to make sure that the isotope does not decay into daughter isotopes that are gamma or neutron emitters. The half-life will determine the length of time that the material will produce energy, but this energy production will decrease with time; these factors must be considered for a particular application.

Although there will be public perception concerns with regards to environmental and safety issues for such devices, there is a precedent for using these materials in common household and office devices such as smoke detectors and illuminated signs. Currently, research efforts associated with small-scale radioactive energy harvesting devices focus on reducing the cost and improving the efficiency of these devices. The atomic batteries have potential applications in MEMS devices, SHM embedded sensing networks, or human medical sensing applications.

\subsection{RF Energy Harvesting}

Another way of supplying power to sensor networks is that of wireless energy transmission. In this case, power is generated elsewhere and transmitted to a sensor node by some form of electromagnetic wave or RF radiation. This concept can utilize two different RF energy sources, ambient or controlled RF sources. Previous studies showed that electronics can be used to efficiently capture ambient radiation sources and convert them to useful electricity. Harrist [145] attempted to charge a cellular phone battery by capturing ambient $915-\mathrm{MHz} \mathrm{RF}$ energy. Although he was not able to fully charge the battery, he observed $4 \mathrm{mV}$ per second charging time from a typical cellular phone battery. Although there are several electronics that may derive their required power from ambient RF sources, the amount of captured energy is extremely low, typically in the range of a few $\mu \mathrm{W}$. Therefore, the technology that has received the most attention is the microwave transmission with controlled or so called "beamed" RF sources. A source antenna transmits microwaves across the atmosphere or space to a receiver, which can either be a typical antenna with rectifying circuitry to convert the microwaves to DC power or a rectenna (rectifying antenna) that integrates the technology to receive and directly convert the microwaves into DC power. 
A pair of excellent survey articles was written to discuss the history of microwave power $[146,147]$. With the use of rectennas, efficiencies in the $50 \%-80 \%$ range for DC to DC conversion have been achieved. Significant testing has also been done across long distances and with $\mathrm{kW}$ power levels [148]. The study showed the feasibility of the wireless energy delivery systems for actuating large devices, including DC motors and piezoelectric Thunder actuators. Briles et al. [149] invented a RF wireless energy delivery system for underground gas or oil recovery pipes. The RF energy is generated on the surface and travels through the conductive pipe, which acts as an antenna or a waveguide. The sensor module in the bottom of the pipe captures this energy and uses it to power the electrical equipment. A schematic of this concept is shown in Figure 5.8. With a $100 \mathrm{~W}$ transmitted power from the surface, it was estimated that around $48 \mathrm{~mW}$ of instant power can be captured after traveling $1.6 \mathrm{~km}$ along pipe.

Current research efforts in RF wireless energy transmission focus on improving the conversion efficiency and attempting to maximize the output power by designing efficient antennas and rectannas. In particular, circular polarized antennas are being implemented in the rectenna design because they avoid the directionality of other antenna designs [150, 151, 152]. An array of rectennas is increasingly used to improve the output power [153] and several new rectenna design schemes are proposed $[154,155]$. Different elements are also used for efficient rectification [156, 157] in attempts to obtain optimum output power, and these research trends are similar to those typically pursued in the energy harvesting arena.

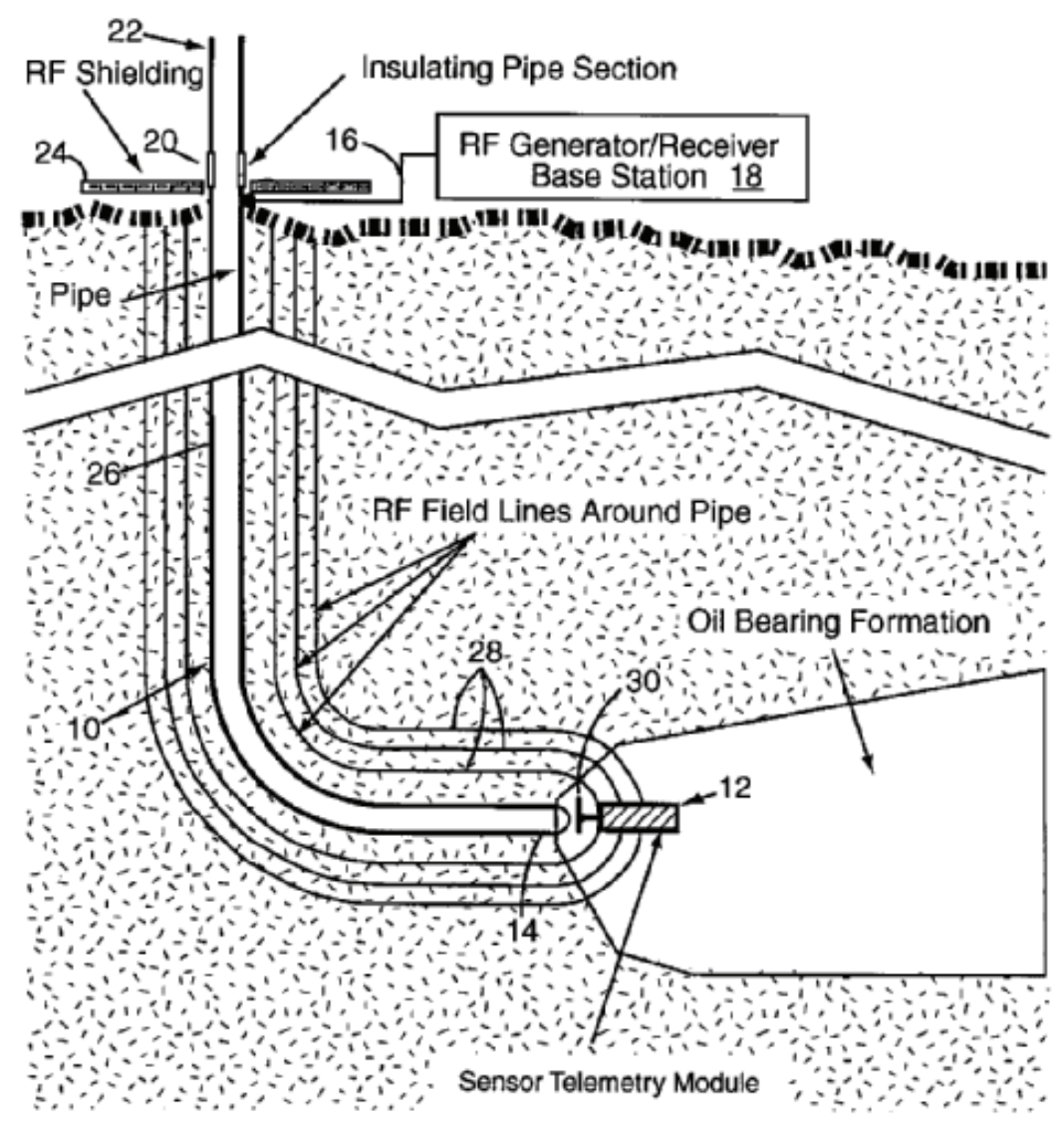

Figure 5.8. A schematic of RF energy delivery system for a down-well pipe. (Source: Briles et al. [149]) 
Originally considered for alleviating the wiring harness in space structures or microscale aerial vehicles and for providing extremely low power as is typically used in RFID tags in the 1to $100-\mu \mathrm{W}$ range, the application of an RF wireless energy transmission system for powering electronics typically used in distributed sensing networks has not been studied substantially in the past. In particular, the application of this technology for SHM sensor nodes in order to alleviate the challenges associated with power supply issues has never been addressed in the literature. A new SHM sensing network proposed by UCSD and LANL researchers that integrates energy transmission between the host and sensor node and uses this energy to both power the sensing circuit and transmit the signal back to the host was described in Section 3.4. The researchers at the Engineering Institute experimentally investigated the RF wireless energy transmission as an alternative power source for wireless SHM sensor nodes [158, 159]. First, a miniaturized active SHM sensor node based on the piezoelectric impedance method was developed, which requires approximately $200 \mathrm{~mW}$ of power to operate. Ten-GHz X-band signals were used as an RF energy propagation frequency in order to facilitate the design of small antennas with high gain. A layout of the RF power delivery system is given in Figure 5.9. Rectenna performance was evaluated based on how quickly the rectenna can supply the required voltage and on how many receive/transmit operations can be successfully completed with this telemetry. In this setup, $1 \mathrm{~W}$ of $\mathrm{X}$-band radiation is transmitted from a horn antenna over a distance of $0.6 \mathrm{~m}$. At the receiving end, the $\mathrm{DC}$ voltage from the rectenna is used to charge up a $0.1 \mathrm{~F}$ supercapacitor. Once a sufficient amount of voltage has built up in the supercapacitor, the stored energy is used to power a MaxStream XBee radio. The experiment results showed that the supercapacitor is able to charge the $0.1 \mathrm{~F}$ supercapacitor to $3.3 \mathrm{~V}$ in $200 \mathrm{~s}$. The average delivered power was estimated at $2.5 \mathrm{~mW}$. This same average power was analytically estimated to be $85 \mathrm{~mW}$ by the one-way radar equation. Possible reasons for the discrepancy include errors in the efficiency estimate for the antennas; variability in the efficiency of the circuit, such as the voltage drops caused by the diodes; electromagnetic reflections off the walls causing destructive interference; and, undoubtedly, an impedance mismatch between the horns and the circuits. Once the capacitor is charged, the radio is able to successfully receive and transmit 256 bytes of data using the asynchronous RS-232 protocol over a distance of $5.2 \mathrm{~m}$. This experiment has shown that RF power delivery can be used to successfully operate the radio, which is the largest power consumer in an SHM sensor node [158].

As illustrated, wireless energy delivery has promise for providing power to SHM or any other long-term wireless sensor nodes. This wireless energy transmission can be used for SHM sensor array interrogation, where both power and activity commands (if desired) are conveyed to the node from a mobile host. For example, an unmanned aerial vehicle whose autopilot and GPS units are programmed to seek out specific coordinates populated by sensors can carry an RF source to activate each sensor. The sensor nodes that are powered by the RF energy will perform the intended measurement, analyze the data on a local computer embedded in the sensor node, and then send the results of the computation to the processor on the mobile host. The advantage of this transmission system is that power does not have to be embedded with the sensing system but rather is transported to the node's vicinity and then wirelessly transmitted to the sensor node. It is anticipated that such a sensor network will have improved reliability and will have inherent advantages when monitoring must be performed in locations that are physically difficult to access. Further, the researchers at the Engineering Institute proposed a hybrid system that couples traditional energy harvesting systems at the node level with the RF energy delivered by the robotic vehicle. 


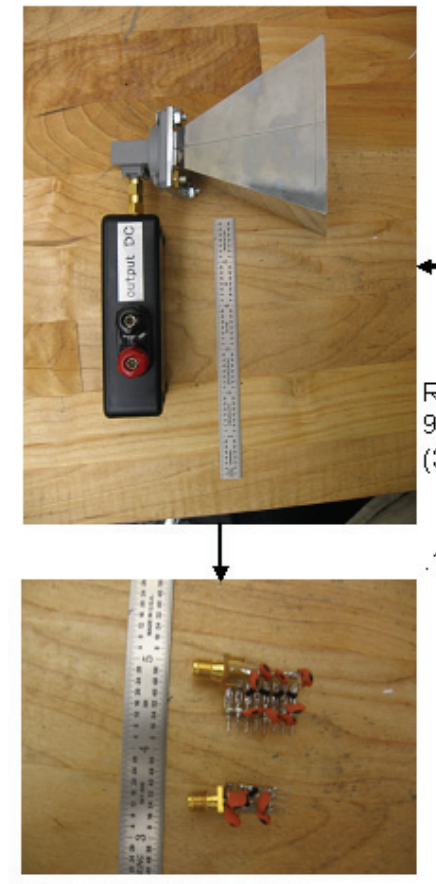

Voltage Rectifier Circuits,

Top, 8X multiply,

Bottom, 4X multiply

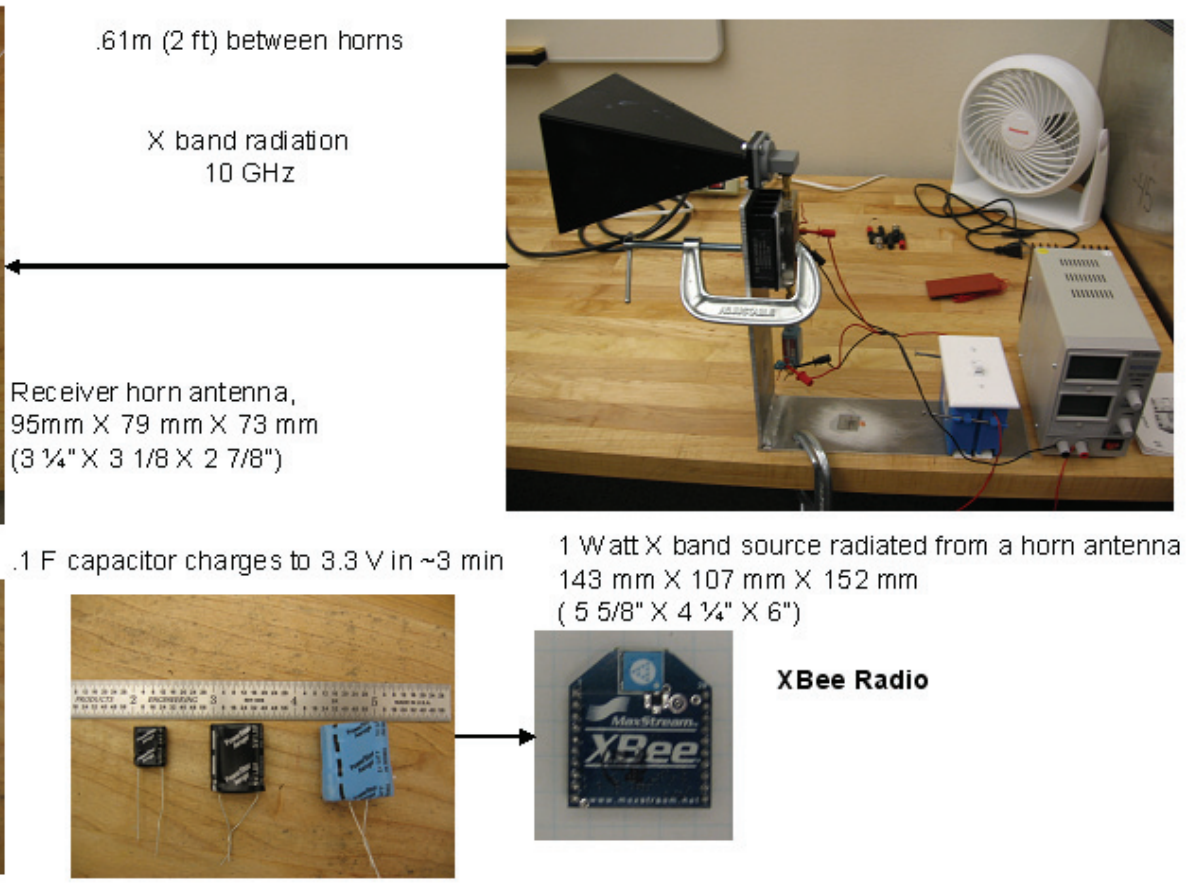

Supercapacitors: . 1F, 1 F, .47 F from left to right

Figure 5.9. RF energy delivery test setup. (Source: Mascarenas [158])

\subsection{Power Conditioning and Storage}

This section will look at research into various types of power storage mediums and different circuits developed to maximize the electric power generated. A typical energy harvesting circuit is shown in Figure 5.10. The AC signal generated by the energy harvesting medium is first fullwave rectified and then stored in a capacitor, where it is subsequently used to charge the battery or any other energy storage medium. Some additional elements, including DC-DC step-down converters, voltage regulators, charge controllers, and charge pump circuits are employed to this circuit to maximize the power flow. All of the circuitry is designed so that the impedance of the harvesting medium and charging circuit can be matched, a more consistent and efficient DC signal can be generated, and the efficient control of energy accumulation can be achieved.

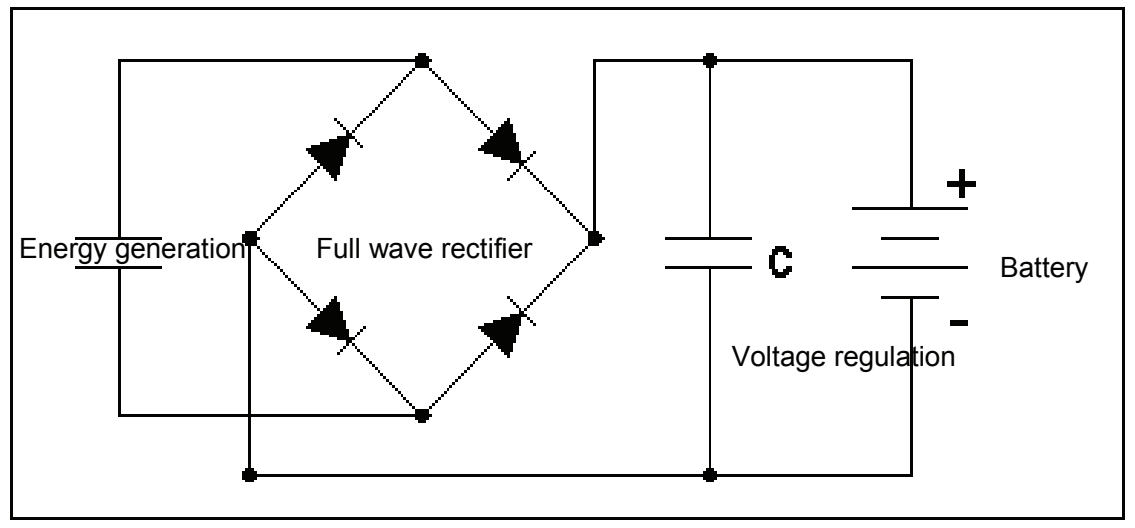

Figure 5.10. Schematic of energy harvesting circuit. 
Much of the research into energy harvesting has dealt with optimizing the power harvesting configuration or developing circuitry to store the energy. However, some researchers have looked into the possibility of using the circuitry to extract more energy from the piezoelectric material. One such study was performed by Kasyap et al. [160], who used the concept that the energy transfer from the piezoelectric to the load is maximized when the impedance of the two are matched to develop a circuit whose impedance could be modified. The authors provide a description of the fly-back converter circuit and the equations needed to set the circuit impedance to the desired value. Ottman et al. [161] studied the use of an adaptive step-down DC-DC converter to maximize the power output from a piezoelectric device. It was found that at very high levels of excitation, the power output could be increased by as much as $400 \%$. However, this study did have a drawback: the additional electronic components required to optimize the power output dissipated energy. This additional circuitry needed an open circuit voltage greater than 10 volts for an increase in the generated power. To overcome this problem, Hofmann et al. [162] modified the circuit by removing the adaptive circuitry and used a fixed switching frequency. However, the improvements made to the circuit now required more than 25 volts open circuit for increased power to be supplied to the load. Furthermore, the level of excitation necessary to produce greater than 25 volts open circuit is far greater than present in any typical vibrating machinery, making the circuitry unrealistic.

One common issue identified for energy harvesting devices is that the amount of energy generated by harvesting mediums is not sufficient to power most electronic devices. Thus, for energy harvesting technology to make its way into the commercial market, methods of accumulating and storing the harvested energy until a sufficient amount can be recovered to power the portable electronics are the key to a successful power harvesting system [32]. One of the first researchers to realize the need for power storage circuitry was Starner [95], who studied the use of piezoelectric materials for harvesting numerous sources of energy around the body, including limb and finger motion. Additionally, the idea of using a capacitor and rechargeable battery for power harvesting was discussed, with some advantages and disadvantages of each listed. This concept was taken a step farther by Umeda et al. [98], who followed their earlier study with an investigation into the use of a capacitor with piezoelectric materials. They theoretically and experimentally tested the circuit in various configurations to determine the optimal design. Shortly after the publication of this work, a power harvesting patent was issued to Kimura [163] for a means of storing the rectified energy from a piezoelectric device in a capacitor. However, a circuit containing only a single capacitor is not sufficient to provide power to other electronic devices without additional circuitry. Therefore, Kymissis et al. [96] developed a piezoelectric system that would harvest the energy lost during walking and use it to power a radio transmitter. Their circuit also used a capacitor as the storage medium, but the additional components allowed it to charge to a desired level before discharging.

While significant headway has been made in the field of energy harvesting, Sodano et al. [32] saw the use of the capacitor as a fundamental problem with the research that had been performed in power storage methods. Because of the poor energy storage characteristics of the capacitor, it could only be used to send out short pulses of energy, which severely limited the number of applications for energy harvesting. Therefore, they investigated the ability to use the energy from the piezoelectric material to recharge a discharged battery. Their study showed that a watch battery could be recharged from a completely discharged state in less than one hour by vibrations consistent in amplitude with those found on a typical vibrating machine. Furthermore, the authors compared this new concept to the more traditional method of storing the energy in a 
capacitor and found that the use of a battery provided more flexibility in the electronics to be powered. Guan and Liao [164] compared the performance of energy storage devices, including conventional capacitors, rechargeable batteries, and supercapacitors, for energy harvesting applications. They concluded that the supercapacitors are more attractive than rechargeable batteries because supercapacitors have higher charge/discharge efficiency, higher adaptability, and much longer lifespans. However, they also have a higher self-discharge rate.

For the RF transmission, the efficiency of the RF energy transfer method lies in the intelligent design of an efficient antenna, along with a circuit capable of converting and amplifying low-amplitude, high-frequency AC signals to DC voltage. The efficiency of the wireless energy harvesting device will be dependent upon the impedance and the shape of the antenna and the impedance of the harvesting circuit. If the impedance of the antenna matches that of the harvesting circuit, the transmitted RF signals will have minimal reflection, thus, a large portion of RF energy will be captured and converted to useful electrical energy. The antenna requires good grounding, and further, the captured energy should be immediately rectified; otherwise, the energy could be easily dissipated as heat, resulting in a poor efficiency. The rectification is a major source of energy loss in harvesting devices. In order to reduce the loss from the rectification diodes, Schottky barrier diodes have been typically used. Compared to other energy generators, TEG does not require a means of rectification because the output of the TEG is a DC signal, which simplifies the associated electronic circuit design.

\subsection{Applications to SHM}

Although the energy harvesting techniques are still in a development stage, several conceptual designs for SHM applications have been proposed. Elvin et al. [165] proposed a selfpowered damage detection sensor using piezoelectric patches. A network of self-powered strain energy sensors were embedded inside a structure, and a moving cart capable of applying a timevarying dynamic load was driven over the structure. The harvesters convert this applied load into electricity and the sensors measure the strain induced by the moving cart, which is also equipped with a receiver and a signal processing unit, as shown in Figure 5.11. The energy generated from the piezo-generator was accumulated in a capacitor. A switch was added to the circuitry to allow the capacitor to charge to a predetermined value of 1.1 volts, at which point the switch would
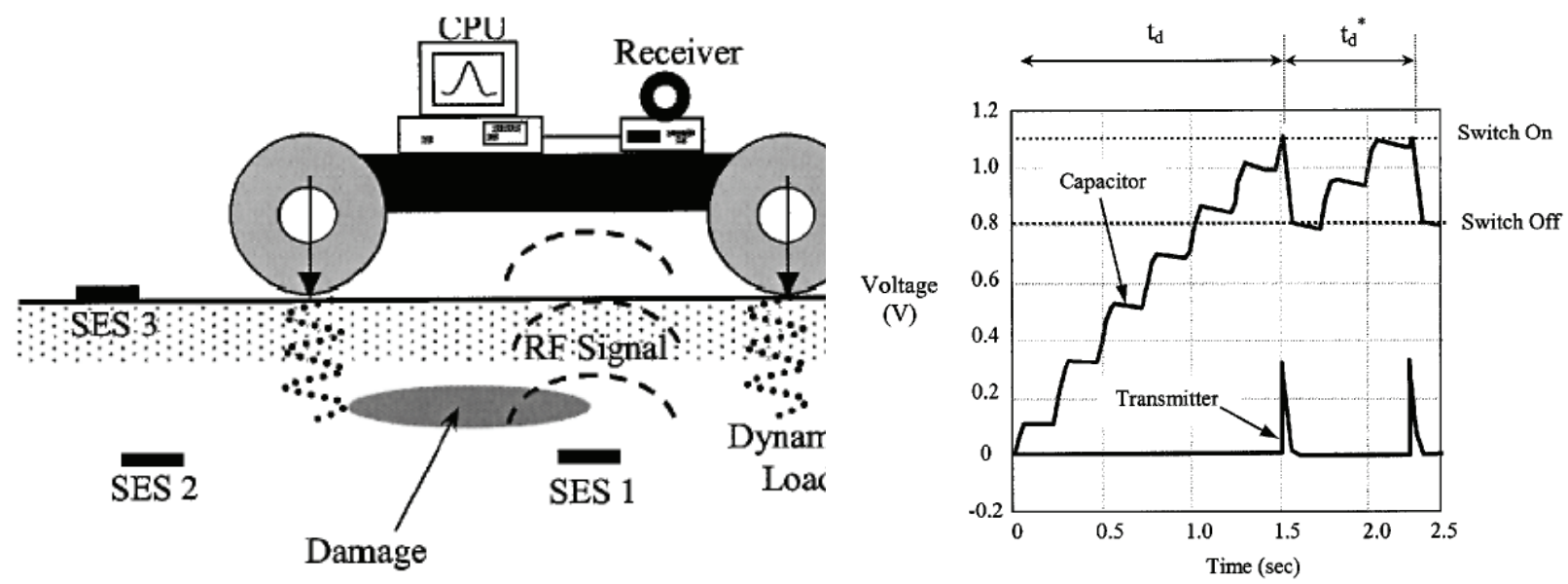

Figure 5.11. Implementation of self-powered sensors for damage detection. (Source: Elvin et al. [165], reprint permission is granted by the Council of the Institution of Mechanical Engineers.) 
open and the capacitor would discharge through the transmitter. Once the capacitor had discharged to a value of 0.8 volts, the switch would close and the capacitor would be allowed to recharge and repeat the process, as shown in Figure 5.11. The operation of the energy harvesting system was found to provide the required energy to power the circuitry and transmit a signal containing information regarding the strain of the beam over a distance of $2 \mathrm{~m}$. James et al. [166] also proposed two prototypes of self-powered systems for condition monitoring applications. The devices, using a low-power accelerometer as a sensor, are powered by a vibration-based electromagnetic generator, which provides a constant power of $2.5 \mathrm{~mW}$. The first system uses a liquid crystal display of the system output, and the second uses an infrared link to transmit the data. However, the systems are not equipped with a local computing capability and only send out the direct sensor readings.

Discenzo et al. [167] developed a prototype self-powered sensor node that performs sensing and local processing and telemeters the result to a central node for pump condition monitoring applications, shown in Figure 5.12. A wireless mote system was integrated with a piezoelectric energy harvesting technique. The device was mounted on an oil pump, which operates $130 \mathrm{~Hz}$, and a cantilever piezoelectric beam tuned to the same frequency was embedded with the sensor node to extracted energy from the pump vibration. The device was installed on an oil tanker for a fourmonth period to measure the vibration data. The maximum power output of $40 \mathrm{~mW}$ was achieved. However, the output power was only $10 \%$ of the amount expected under these operating conditions. The low power output was believed to come from the slight discrepancy in the excitation frequency and the piezoelectric device's resonant frequency. The authors suggested the development of an adaptive-tuning harvester to accommodate uncertainties in the environment and in equipment operation.

Pfeifer et al. [168] investigated the development of self-powered sensor tags that can be used to monitor the health of a structure. A microcontroller, which consumes $40 \mu \mathrm{W}$ to operate, was powered by a piezoelectric patch $(7.5 \times 5 \mathrm{~cm})$. Once powered, the microcontroller operates the sensor array, performs the local computing, and saves the results of computation into a RFID tag. By storing the data in nonvolatile memory, the data can be retrieved by a mobile host even if the sensor node does not have enough power to operate. In a laboratory setting, the piezoelectric harvester can deliver enough energy to the microcontroller for 17 seconds of operation. A schematic of this system is shown in Figure 5.13.

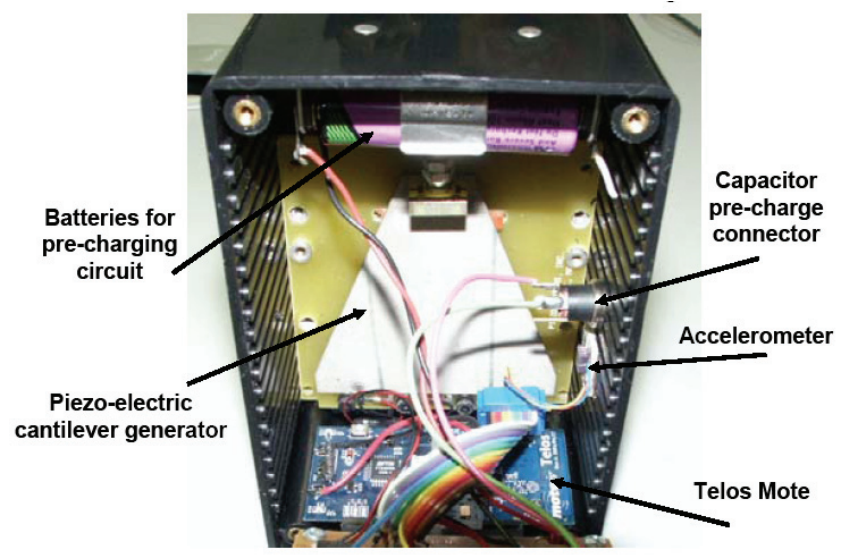

Figure 5.12. Self-powered sensor node.

(Source: Discenzo et al. [167], reprinted with permission of Sound and Vibration Magazine.) 


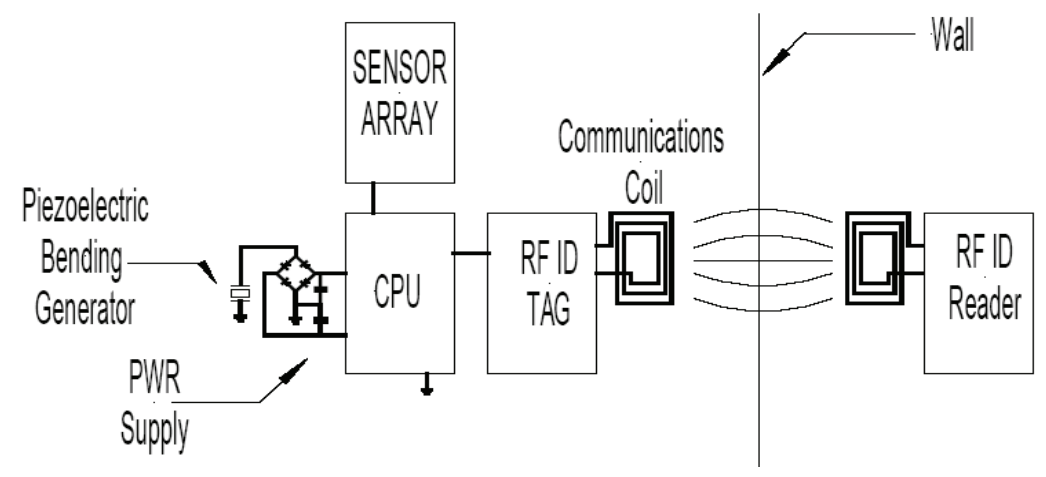

Figure 5.13. Diagram of self-powered sensor system. (Source: Pfeifer et al. [168])

Ha and Chang [169] assessed the suitability and efficiency of energy harvesting techniques for an SHM system based on a network of piezoelectric sensors and actuators. They concluded that total power requirements of the piezoelectric Lamb-wave based SHM system far exceed the current energy harvesting capability. However, they suggested that a passive sensing system, which uses passive acoustic emission and detects an accidental impact event, would be a good candidate for energy harvesting technology because of the low power requirements and very low duty-cycle.

\subsection{Conclusion}

Energy harvesting is slowly coming into full view of the SHM and more general sensing network communities. With continual advances in wireless sensor/actuator technologies, improved signal processing techniques, and the continued development of power efficient electronics, energy harvesting will continue to attract the attention of researchers and field engineers. However, it should be emphasized that the energy harvesting still remains in its infancy and only a few successful examples are in practice. Still, a tremendous research effort is required to convert, optimize, and accumulate the necessary amount of energy to power such electronics.

\section{FUTURE RESEARCH NEEDS AND CHALLENGES}

This section outlines future research areas for energy harvesting in order to transition the current state-of-the-art to full-scale deployment in the current practice of SHM and sensing networks.

As identified, the major limitations facing researchers in the field of energy harvesting revolve around the fact that the energy generated by harvesting devices is far too small to directly power most electronics. Therefore, efficient and innovative methods of storing electrical energy are the key technologies that will allow energy harvesting to become a source of power for electronics and wireless sensors. Energy storage mediums, including rechargeable batteries, capacitors, and ultracapacitors, should be carefully selected depending on a specific application. For instance, ultracapacitors, which have extremely high energy storage capacity, could be used as effectively as batteries for many low-power applications. Ultracapacitors possess the ability to deliver bursts of high power, can be recharged rapidly from any energy source, and are capable 
over 600,000 charge cycles [84]. Furthermore, they are robust and rigorous under harsh environmental conditions. Another exciting possibility is the emerging technology of flexible, thin-film batteries [170] or power-fiber batteries [171] that can be fully integrated into energy harvesting mediums, forming the concept of structural batteries or harvesting batteries. For instance, $5-\times 5$-cm patches consisting of 300 power fibers are projected to store $25 \mathrm{mWh}$ of energy [171]. This novel design will reduce the mass and volume of the energy supply for microscale sensing systems where the dimension constraints are critical. It can be also envisioned that, if sufficient ambient energy is available, multiple recharging circuits for two or more batteries (or capacitors) could be installed, allowing one battery to be in the charging stage while the other is in the operational stage, in an effort to provide a constant and virtually endless power supply to the system. It should also be emphasized that, for any storage medium, the duty cycle of the application must be considered, as this factor drastically changes the design parameters and associated electronics. It is necessary to match the duty cycle to the time required to store enough energy until it is needed by electronics. It is also worthwhile pursuing a hybrid system that integrates energy harvesters with a RF wireless energy delivery system. The energy delivery system can be used to convey activity commands or it may provide additional energy if the harvester does not have enough energy to operate a sensor node.

Research on energy harvesting materials has focused mainly on determining the extent of power capable of being generated rather than investigating applications and uses of the harvested energy. The practical applications for energy harvesting systems, such as wireless self-powered SHM sensing networks, must be clearly identified with emphasis on power management issues. Application-specific, design-oriented approaches are needed to help with the practical use of these technologies. It was suggested that the biggest roadblock for using energy harvesting devices is the lack of clear design guidelines that help determine how to characterize the ambient energy, what circuits and storage devices are best for a given application, and what strategies are best to integrate the harvesting devices into embedded sensor units. Additionally, the intended location of the energy harvesting system must be identified so that its placement can be optimized and the excitation range realized to allow for tuning the energy harvesting device. Developing such guidelines demands substantial research efforts to define the key parameters and predictive models affecting efficient energy harvesting.

Reliability is an essential requirement for any energy source. Because many vibration-based harvesters are designed to operate at their resonances, the systems will be inherently unstable after long operation cycles. Also, energy sources for field use should be able to withstand harsh environmental conditions. The reliability and robustness must be proved before the energy harvesting techniques can be used in practice.

Few studies addressed the integrated use of available energy harvesting devices. Each energy harvesting scheme needs to be compared precisely to the other methods and, if necessary, integrated to maximize the energy generation under a given environmental condition. To realize this integration, a general standard should be established to address the technical capabilities of each energy source for system integrators so that they can easily assemble components for final design, as suggested by Fry et al. [84]. 
The goal of maximizing the amount of harvested energy involves several factors, including electronics optimization, characterization of the available ambient energy, selection and configuration of energy harvesting materials, and integration with storage mechanisms. Few studies have addressed these issues in an integrated manner from the multidisciplinary engineering perspective.

Finally, it has been identified that, although several energy harvesting devices have been developed and fabricated as prototypes, the performance of these techniques in real operational environments needs to be verified and validated.

\section{SUMMARY}

This report is the first in an anticipated series of reports that will be generated from the LANL/UCSD Engineering Institute's annual workshops. The plan is to have the focus of these workshops rotate on an annual basis between topics related to sensing and data acquisition, data interrogation, and predictive modeling as these topics relate to structural health monitoring and damage prognosis. The material summarized herein was developed based on information exchanges during the first 2.5-day workshop that took place from June 28-30, 2005, at Los Alamos National Laboratory. This workshop focused on sensing and data acquisition and specifically addressed energy harvesting for embedded structural health monitoring (SHM) sensing systems.

A statistical pattern recognition paradigm for SHM is first presented and the concept of energy harvesting for embedded sensing systems is addressed with respect to the data acquisition portion of this paradigm. Next, various existing and emerging sensing modalities used for SHM and their respective power requirements are summarized. Currently, these systems almost exclusively measure kinematic quantities. It was pointed out that more recently active approaches to sensing are being developed that couple local actuation capabilities with the sensing system.

Next, sensor network paradigms that have been employed for SHM were discussed along with emerging network paradigms that are being considered for future SHM applications. Currently, wired networks are still the most prevalent systems employed for SHM. Alternatively, recent SHM research is focusing on the use of wireless sensor nodes that are primarily powered by batteries. These networks are being developed to accommodate active as well as traditional passive sensing. It is envisioned that future SHM sensing systems will be hybrid systems that couple local active sensing to identify the onset of damage with passive global sensing that will assess the impact of that damage on system-level performance. Clearly, to have a truly embedded sensing system that requires relatively little maintenance, one needs to replace the battery, which can be depleted on a fairly short time scale, with an energy harvesting system.

The sensor network summary was followed by a detailed discussion of energy requirements for the various components of the network. Here not only the energy demands of the various components making up the sensor network are considered, but the duty cycle associated with a particular sensing application is also addressed. Included in this discussion are two approaches that enable the system to save power. These approaches are dynamic power management and dynamics voltage scaling. Because both methods take advantage of component idleness, they can be combined into a single power management framework. It is concluded that the relative 
strengths of these two power management methods dictate that a hybrid approach will yield a more power-efficient embedded sensing system.

Various approaches to energy harvesting and energy storage are then discussed and limitations associated with the current technology are addressed. The approaches can be grouped into those that convert mechanical energy into electrical energy and those that convert thermal energy into electrical energy. This discussion also addresses current energy harvesting applications and system integration issues, with a summary of applications to SHM sensing systems. While it is noted that there is tremendous research into the development of energy harvesting schemes for large-scale alternative sources such as wind turbines and solar cells and that these large-scale systems have made the transition from research to commercial products, energy harvesting for embedded sensing systems is still in it infancy. Also, there is no clearly defined design process to develop such energy harvesting for embedded sensing systems.

The report concludes by defining some future research directions and possible technology demonstrations that are aimed at transitioning the concept of energy harvesting for embedded SHM sensing systems from laboratory research to field-deployed engineering prototypes. In this regard, the Engineering Institute is leading the development of an RF energy delivery system that will bring power to the sensor nodes using an unmanned aerial vehicle.

\section{REFERENCES}

1. K. Worden and J.M. Dulieu-Barton, 2004, "An Overview of Intelligent Fault Detection in Systems and Structures," International Journal of Structural Health Monitoring, 3(1) pp. 85-98.

2. C.R. Farrar, S.W. Doebling, and D.A. Nix, 2001, "Vibration-Based Structural Damage Identification," Philosophical Transactions of the Royal Society: Mathematical, Physical \& Engineering Sciences, 359(1778) pp. 131-149.

3. H. Sohn, C.R. Farrar, F.M. Hemez, D.D. Shunk, D.W. Stinemates, and B. R. Nadler, 2004, "A Review of Structural Health Monitoring Literature from 1996-2001," Los Alamos National Laboratory report LA-13976-MS.

4. S.W. Doebling, C.R. Farrar, M.B. Prime, and D. Shevitz, 1996, "Damage Identification and Health Monitoring of Structural and Mechanical Systems From Changes in their Vibration Characteristics: A literature Review," Los Alamos National Laboratory report LA-13070-MS.

5. G. Park, H. Sohn, C.R. Farrar, and D.J. Inman, 2003, “Overview of Piezoelectric ImpedanceBased Health Monitoring and Path Forward," Shock and Vibration Digest, 35(6) pp. 451-463.

6. H. Sohn, G. Park, J.R. Wait, N. Limback, and C.R. Farrar, 2004, "Wavelet-Based Signal Processing for Detecting Delamination in Composite Plates," Smart Material and Structures, 13(1) pp. 153-160.

7. S.S. Kessler, S.M. Spearing, and C. Soutis, 2002, "Damage Detection in Composite Materials Using Lamb Wave Methods," Smart Materials and Structures, 11(2) pp. 269-278.

8. J.B. Ihn and F.K. Chang, 2004, "Detection and Monitoring of Hidden Fatigue Crack Growth Using a Built-in Piezoelectric Sensor/Actuator Network: II. Validation Using Riveted Joints and Repair Patches," Smart Materials and Structures, 13(3) pp. 621-630. 
9. C.R. Farrar, H. Sohn, and K. Worden, 2001, "Data Normalization: A Key to Structural Health Monitoring," Proc. of the Third International Structural Health Monitoring Workshop, Stanford, CA. DEStech Publications, Inc., Lancaster, PA pp. 1229-1238.

10. C.-Y. Chong and S.P. Kumar, 2003, "Sensor Networks: Evolution, Opportunities, and Challenges," Proc. of the IEEE, 91(8), pp.1247-1256.

11. G. Kulwanoski and J. Schnellinger, 2004 "The Principles of Piezoelectric Accelerometers," Sensors Magazine, 21(2), pp. 27-33.

12. R. Howe, 1995, "Polysilicon Integrated Microsystems: Technologies and Applications," Proc. of Int. Solid-State Sensors and Actuators Conference, Transducers'95, pp. 43-46.

13. J. Bernstein, 2003, “An Overview of MEMS Inertial Sensing,” Sensors, 20 (2), pp. 14-21

14. M.D. Todd, 2004, "Optical-Based Sensing," in Damage Prognosis, Edited by D.J. Inman, C.R. Farrar, V. Lopes, and V. Steffen, pp. 343-362, John Wiley and Sons, Inc., pp. 343-362.

15. M. D. Todd, G. A. Johnson, and B. L. Althouse, 2001, "A Novel Bragg Grating Sensor Interrogation System Utilizing a Scanning Filter, a Mach-Zehnder Interferometer, and a $3 \times 3$ Coupler," Measurement Science and Technology, 12(7) pp. 771-777.

16. S. E. Park and T. R. Shrout, 1997, "Ultrahigh Strain and Piezoelectric Behavior in Relaxor Based Ferroelectric Single Crystals," Journal of Applied Physics, 82 pp. 1804-1811.

17. V. Giurgiutiu, Z. Chaudry, and C. A. Roger, 1997, "Design of Displacement Amplified Induced Strain Actuators for Maximum Energy Output," ASME Journal of Mechanical Design, 119 pp. 511-517.

18. P.D. Wilcox, M.J.S. Lowe, and P. Cawley, 2005, "Omni-Directional Guided Wave Inspection of Large Metallic Plate Structures Using an EMAT Array," IEEE Trans. on Ultrason. Ferroelec. and Freq. Cont. 52, pp. 653-665.

19. V. Giurgiutiu, A. Zagrai, J.J. Bao, 2002 "Piezoelectric Wafer Embedded Active Sensors for Aging Aircraft Structural Health Monitoring," International Journal of Structural Health Monitoring, 1, pp. 41-61.

20. C. Niezrecki, D. Brei, S. Balakrishnan, and A. Moskalik, 2001, "Piezoelectric Actuation: State of the Art," Shock and Vibration Digest, 33(4) pp. 269-280.

21. Y.Q. Ni, B.S. Wang, and J.M. Ko, 2001, "Simulation Studies of Damage Location in Tsing Ma Bridge Deck," Proc., Nondestructive Evaluation of Highways, Utilities, and Pipelines IV, SPIE, Bellingham, Wash., pp. 312-323.

22. M. Lin, X. Qing, A. Kumar, and S. Beard, 2001, "SMART Layer and SMART Suitcase for Structural Health Monitoring Applications," Proc. of SPIE Smart Structures and Materials Conf., Vol. 4332 pp. 98-106.

23. N.A. Tanner, J.R. Wait, C.R. Farrar, and H. Sohn, 2003, "Structural Health Monitoring Using Modular Wireless Sensors," Journal of Intelligent Material Systems and Structures, 14(1) pp. 43-56. 
24. J.P. Lynch, K.H. Law, A.S. Kiremidjian, E. Carryer, T.W. Kenny, A. Partridge, and A. Sundararajan, 2002, "Validation of a Wireless Modular Monitoring System for Structures," SPIE 9th Annual International Symposium on Smart Structures and Materials, San Diego, CA, USA, March 17-21, 2002.

25. B.F. Spencer, M.E. Ruiz-Sandoval, and N. Kurata, 2004, "Smart Sensing Technology: Opportunities and Challenges," Structural Control and Health Monitoring, 11(4) pp. 349-368.

26. C.R. Farrar, D.W. Allen, G. Park, S. Ball, and M.P. Masquelier, 2006, "Coupling Sensing Hardware with Data Interrogation Software for Structural Health Monitoring," Shock and Vibration, 13 (4), pp. 519-530.

27. J.P. Lynch, A. Partridge, K.H. Law, T.W. Kenny, A.S. Kiremidjian, and E. Carryer, 2003, "Design of a Piezoresistive MEMS-Based Accelerometer for Integration with a Wireless Sensing Unit for Structural Monitoring," ASCE Journal of Aerospace Engineering, 16 pp. 108-114.

28. J.P. Lynch and K.J. Loh, 2006, "A Summary Review of Wireless Sensors and Sensor Networks for Structural Health Monitoring," The Shock and Vibration Digest, 38, pp. 91-128.

29. J.R. Dove, G. Park, and C.R. Farrar, 2005, "Hardware Design of Hierarchal Active-Sensing Networks for Structural Health Monitoring," Smart Materials and Structures, 15, pp. 139-146.

30. G. Park, C.R. Farrar, C.A. Rutherford, and A.N. Robertson, 2006, "Piezoelectric Active Sensor Self-Diagnostics Using Electrical Admittance Measurements," ASME Journal of Vibrations and Acoustics, 128, pp. 469-476.

31. G. Kerschen, P.D. Boe, J. Golinval, and K. Worden, 2005, "Sensor Validation Using Principal Component Analysis," Smart Materials and Structures, 14(1) pp. 36-42.

32. H.A. Sodano, D.J. Inman, and G. Park, 2004, "A Review of Power Harvesting from Vibration Using Piezoelectric Materials," The Shock and Vibration Digest, 36(3) pp. 197-205.

33. M. Srivastave, 2001, Sensors Tutorial in $7^{\text {th }}$ Annual Intl. Conf. on Mobile Computing and Networks (MOBICOM'01).

34. A. Chandrakasan and R. Brodersen, 1995, Low power digital CMOS design, Kluwer.

35. J. Rabaey and M. Pedram (Editors), 1996, Low power design methodologies, Kluwer.

36. W. Nabel and J. Mermet (Editors), 1997, Lower power design in deep submicron electronics, Kluwer.

37. C. Ellis, 1999, "The Case for Higher-Level Power Management," 7th IEEE Workshop on Hot Topics in Operating Systems, pp. 162-167.

38. L. Benini and G. De Micheli, 1997, Dynamic Power Management: Design Techniques and CAD Tools, Kluwer.

39. M.B. Srivastava, A.P. Chandrakasan, and R.W. Brodersen, 1996, "Predictive System Shutdown and Other Architectural Techniques for Energy Efficient Programmable Computation," IEEE Trans. VLSI Systems, 4(1) pp. 42-55.

40. C.-H. Hwang and A. Wu, 1997, “A Predictive System Shutdown Method for Energy Saving of Event-Driven Computation," Int. Conf. on Computer Aided Design, pp. 28-32. 
41. L.S. Brakmo, D.A. Wallach, and M.A. Viredaz, 2004, " $\mu$ Sleep: A Technique for Reducing Energy Consumption in Handheld Devices," Proc. USENIX/ACM Int. Conf. Mobile Systems, Applications, \& Services, pp. 12-22.

42. N. Kamijoh, T. Inoue, C.M. Olsen, M.T. Raghunath, and C. Narayanaswami, 2001, "Energy Tradeoffs in the IBM Wristwatch Computer," Proc. IEEE Int. Symp. Wearable Computers, pp. 133-140.

43. K. Li, R. Kumpf, P. Horton, and T.E. Anderson, 1994, "A Quantitative Analysis of Disk Drive Power Management in Portable Computers," Proc. USENIX Winter Tech. Conf., pp. 279-291.

44. A. Karlin, M. Manesse, L. McGeoch, and S. Owicki, 1994, "Competitive Randomized Algorithms for Nonuniform Problems," Algorithmica, pp. 542-571.

45. F. Douglis, P. Krishnan, and B.N. Bershad, 1995, “Adaptive Disk Spin-Down Policies for Mobile Computers," Proc. 2nd USENIX Symp. Mobile \& Location-Independent Computing, pp. 121-137.

46. D.P. Helmbold, D.D.E. Long, and B. Sherrod, 1996, "A Dynamic Disk Spin-Down Technique for Mobile Computing," Proc. ACM Ann. Int. Conf. Mobile Computing \& Networking, pp. 130-142.

47. P. Krishnan, P. Long, and J. Vitter, 1999, “Adaptive Disk Spindown via Optimal Rent-toBuy in Probabilistic Environments," Algorithmica, 23(1) pp. 31-56.

48. S. Irani, S. Shukla, and R. Gupta, 2003, “Online Strategies for Dynamic Power Management in Systems with Multiple Power-Saving States," ACM Trans. Embedded Computing Systems, 2(3) pp. 325-346.

49. D. Ramanathan, S. Irani, and R. Gupta, 2000, "Latency Effects of System Level Power Management Algorithms," Proc. IEEE/ACM Int. Conf. Computer-Aided Design, pp 350-356.

50. R. Golding, P. Bosch, and J. Wilkes, 1995, "Idleness is not Sloth," in Proc. USENIX Winter Tech. Conf., pp. 201-212.

51. M.B. Srivastava, A.P. Chandrakasan, and R.W. Brodersen, 1996, "Predictive System Shutdown and Other Architectural Techniques for Energy Efficient Programmable Computation," IEEE Trans. VLSI Systems, 4(1) pp. 42-55.

52. C.-H. Hwang and A. Wu, 1997, “A Predictive System Shutdown Method for Energy Saving of Event-Driven Computation," Int. Conf. on Computer Aided Design, pp. 28-32.

53. L. Zhong and N.K. Jha, 2004 "Dynamic Power Optimization for Interactive Systems," in Proc. Int. Conf. VLSI Design, pp. 1041-1047.

54. T. Simunic, S. Boyd, and P. Glynn, 2004, "Managing Power Consumption in Networks on Chips," IEEE Transactions on VLSI, pp. 96-107.

55. L. Benini, G. Paleologo, A. Bogliolo, and G. DeMicheli, 1999, "Policy Optimization for Dynamic Power Management," IEEE Transactions on Computer-Aided Design, 18(6), pp. 813-833 
56. E. Chung, L. Benini, and G. De Micheli, 1999, "Dynamic Power Management for Nonstationary Service Requests," Design, Automation and Test in Europe, pp. 77-81.

57. Q. Qiu and M. Pedram, 1999, "Dynamic Power Management Based on Continuous-Time Markov Decision Processes,” Design Automation Conf., pp. 555-561.

58. T. Simunic, L. Benini, P. Glynn, and G. De Micheli, 2001, "Event-Driven Power Management," IEEE Transactions on CAD, pp.840-857.

59. Q. Qiu and M. Pedram, 2000, "Dynamic Power Management of Complex Systems Using Generalized Stochastic Petri Nets," Design Automation Conf. pp. 352-356.

60. V. Paxson and S. Floyd, 1995, "Wide Area Traffic: The Failure of Poisson Modeling," IEEE Transactions on Networking, 3(3), pp. 226-244.

61. S. Shukla and R. Gupta, 2001, "A Model Checking Approach to Evaluating System Level Power Management for Embedded Systems," Proceedings of $6^{\text {th }}$ IEEE Int. High-Level Design Validation and Test Workshop, pp. 53-57.

62. Http://www.acpi.info/DOWNLOADS/ACPIspec30b.pdf, last accessed on 01/25/2007.

63. Y.-H. Lu, L. Benini, and G. De Micheli, 2002, "Power-Aware Operating Systems for Interactive Systems," IEEE Trans. VLSI Systems, 10(2) pp.119-134.

64. J. Flinn and M. Satyanarayanan, 2002, "Managing Battery Lifetime with Energy-Aware Adaptation," ACM Trans. Computer Systems, 22(2) pp.137-179.

65. W. Yuan, K. Nahrstedt, S. Adve, D. Jones, and R. Kravets, 2006, "GRACE-1: Cross-Layer Adaptation for Multimedia Quality and Battery Energy," IEEE Trans. Mobile Computing, 5(7), pp. 799-815.

66. D. Bertozzi, L. Benini, and B. Ricco, 2002, "Power Aware Network Interface Management for Streaming Multimedia," Proc. IEEE Wireless Communication Network Conf., pp. 926-930, Orlando, FL.

67. A. E. Papathanasiou and M.L. Scott, 2004, "Energy Efficient Prefetching and Caching," in Proc. USENIX Ann. Tech. Conf., pp. 255-268.

68. L. Cai and Y.-H. Lu, 2005, "Energy Management Using Buffer Memory for Streaming Data," IEEE Trans. Computer-Aided Design of IC \& Systems, 24(2) pp.141-152.

69. J. Kim and T. Simunic Rosing, 2006, "Power-Aware Resource Management Techniques for Low-Power Embedded Systems," Handbook of Real-time Embedded Systems, Edited by I. Lee, J. Y-T. Leung, S. Son, CRC Press, in press.

70. Y. Shin, K. Choi, and T. Sakurai, 2000, "Power Optimization of Real-Time Embedded Systems on Variable Speed Processors," Proc. of the Int. Conf. on Computer-Aided Design, pp. $365-368$.

71. F. Gruian, 2001, "Hard Real-Time Scheduling Using Stochastic Data and DVS Processors," in Proc. of Int. Symposium on Low Power Electronics and Design, pp. 46-51. 
72. W. Kim, J. Kim, and S.L. Min, 2002, “A Dynamic Voltage Scaling Algorithm for DynamicPriority Hard Real-Time Systems Using Slack Time Analysis," in Proc. of Design, Automation and Test in Europe (DATE'02), pp. 788-794.

73. H. Aydin, R. Melhem, D. Mosse, and P.M. Alvarez, 2001, "Dynamic and Aggressive Scheduling Techniques for Power-Aware Real-Time Systems," in Proc. of IEEE Real-Time Systems Symposium, pp. 95-105.

74. S. Lee and T. Sakurai, 2000, "Run-Time Voltage Hopping for Low-Power Real-Time Systems," in Proc. of Design Automation Conference, pp. 806-809.

75. D. Mosse, H. Aydin, B. Childers, and R. Melhem, 2000, "Compiler-Assisted Dynamic Power-Aware Scheduling for Real-Time Applications," in Proc. of Workshop on Compiler and OS for Low Power (http://www.cs.pitt.edu/PARTS/papers/COLP00_mosse.pdf, last accessed on 01/25/2007).

76. N. AbouGhazaleh, B. Childers, D. Mosse, R. Melhem, and M. Craven, 2002, "Energy Management for Real-Time Embedded Applications with Compiler Support," in Proc. of Conference on Language, Compiler, and Tool Support for Embedded Systems, pp. 238-246.

77. D. Shin, J. Kim, and S. Lee, 2001, "Intra-Task Voltage Scheduling for Low-Energy Hard Real-Time Applications," IEEE Design and Test of Computers, 18(2), pp. 20-30.

78. C-H. Hsu and U. Kremer, 2003, "The Design, Implementation, and Evaluation of a Compiler Algorithm for CPU Energy Reduction," in Proc. of ACM SIGPLAN Conference on Programming Languages, Design, and Implementation, pp. 38-48.

79. K. Choi, R. Soma, and M. Pedram, 2005, "Fine-Grained Dynamic Voltage and Frequency Scaling for Precise Energy and Performance Trade-Off Based on the Ratio of Off-Chip Access to on-Chip Computation Times," IEEE Transactions on Computer Aided Design, 24(1), pp. 18-28.

80. A. Weissel and F. Bellosa, 2002, "Process Cruise Control: Event-Driven Clock Scaling for Dynamic Power Management," in Proc. of International Conference on Compilers, Architecture, and Synthesis for Embedded Systems, pp. 238-246.

81. J. R. Lorch and A.J. Smith, 2001, "Improving Dynamic Voltage Scaling Algorithms with PACE," Proc. Of ACM SIGMETRICS Conference, pp. 50-61.

82. F. Gruian, 2001, "Hard Real-Time Scheduling Using Stochastic Data and DVS Processors," in Proc. of International Symposium on Low Power Electronics and Design, pp. 46-51.

83. D. Shin, W. Kim, J. Jeon, and J. Kim, 2003, "SimDVS: an Integrated Simulation Environment for Performance Evaluation of Dynamic Voltage Scaling Algorithms," Lecture Notes in Computer Science, 2325, pp. 141-156.

84. D.N. Fry, D.E. Holcomb, J.K. Munro, L.C. Oakes, and M.J. Maston, 1997, Compact Portable Electric Power Sources, Oak Ridge National Laboratory report, ORNL/TM-13360.

85. P. Glynne-Jones and N.M. White, 2001, "Self-Powered Systems: A Review of Energy Sources," Sensor Review, 21, pp. 91-97. 
86. S.J. Roundy, 2003 Energy Scavenging for Wireless Sensor Nodes with a Focus on Vibration to Electricity Conversion, Ph.D. Dissertation, Department of Mechanical Engineering, University of California, Berkeley.

87. M.A. Qiwai, J.P. Thomas, J.C. Kellogg, and J. Baucom, 2004, "Energy Harvesting Concepts for Small Electric Unmanned Systems," Proc. of SPIE, 5387, pp. 84-95.

88. L. Mateu and F. Moll, 2005, "Review of Energy Harvesting Techniques and Applications for Microelectronics," Proc. of SPIE, 5837, pp. 359-373.

89. J.A. Paradiso and T. Starner, 2005, "Energy Scavenging for Mobile and Wireless Electronics," IEEE Pervasive Computing, 4, pp.18-27.

90. M. Stordeur and I. Stark, 1997, "Low Power Thermoelectric Generator-Self-Sufficient Energy Supply for Micro Systems," Proc. of 16th International Conference on Thermoelectrics, pp. 575-577.

91. D.J. Inman and H.H. Cudney, 2000, Structural and Machine Design Using Piezoceramic Materials: a Guide for Structural Design Engineers, Final Report NASA Langley Grant NAG-1-1998.

92. J. Sirohi and I. Chopra, 2000, "Fundamental Understanding of Piezoelectric Strain Sensors," Journal of Intelligent Material Systems and Structures, 11, pp. 246-257.

93. N.E. duToit, B.L. Wardle, and S.G. Kim, 2005 "Design Considerations for MEMS-Scale Piezoelectric Mechanical Vibration Energy Harvesters," Integrated Ferroelectrics, 71, pp. 21-160.

94. E. Hausler and E. Stein, 1984, "Implantable Physiological Power Supply with PVDF Film," Ferroelectrics, 60, pp. 277-282.

95. T. Starner, 1996, "Human-Powered Wearable Computing," IBM Systems Journal, 35, pp. $18-628$.

96. J. Kymissis, C. Kendall, J. Paradiso, and N. Gershenfeld, 1998, "Parasitic Power Harvesting in Shoes," Proceedings of 2nd IEEE International Symposium on Wearable Computers, October 19-20th, Pittsburg, PA, pp. 132-139.

97. M.J. Ramsey, and W.W. Clark, 2001, "Piezoelectric Energy Harvesting for Bio MEMS Applications," Proc. of SPIE, 4332, Newport Beach, CA, pp. 29-438.

98. M. Umeda, K. Nakamura, and S. Ueha, 1996, “Analysis of Transformation of Mechanical Impact Energy to Electrical Energy Using a Piezoelectric Vibrator," Japanese Journal of Applied Physics, 35, pp. 3267-3273.

99. S. Roundy, P. Wright, and J. Rabaey, 2003, “A Study of Low Level Vibrations as a Power Source for Wireless Sensor Nodes," Computer Communications, 23, pp.1131-1144.

100. http://www.oceanpowertechnologies.com/.

101. H.A. Sodano, D.J. Inman, and G. Park, 2005, "Comparison of Piezoelectric Energy Harvesting Devices for Recharging Batteries," Journal of Intelligent Material Systems and Structures, 16, pp. 799-807. 
102. H.A. Sodano, G. Park, and D.J. Inman, 2004. "Estimation of Electric Charge Output for Piezoelectric Energy Harvesting," Strain, 40, pp. 49-58.

103. F. Lu, H.P. Lee, and S.P. Lim, 2004, "Modeling and Analysis of Micro Piezoelectric Power Generators for Micro-Electromechanical-Systems Applications," Smart Materials and Structures, 13, pp. 57-63.

104. J. Twiefel, B. Richter, T. Hemsel, and J. Wallaschek, 2006, "Model-based Design of Piezoelectric Energy Harvesting Systems,” Proc. of SPIE, 6169, pp. 09.1-09.10.

105. S. Roundy and P.K. Wright, 2004, "A Piezoelectric Vibration Based Generator for Wireless Electronics," Smart Materials and Structures, 13, pp. 1131-1142.

106. S. Kim, W.W. Clark, and Q.M. Wang, 2005, “ Piezoelectric Energy Harvesting with a Clamped Circular Plate: Analysis," Journal of Intelligent Material Systems and Structures, 16, pp. 847-854.

107. J.W. Sohn, S.B. Choi, and D.Y. Lee, 2005, "An Investigation on Piezoelectric Energy Harvesting for MEMS Power Sources," Proc. of the Institution of Mechanical Engineers, Part C-Journal of Mechanical Engineering Science, 219, pp. 429-436.

108. M. Goldfarb and L.D. Jones, 1999, "On the Efficiency of Electric Power Generation with Piezoelectric Ceramic," ASME Journal of Dynamic Systems, Measurement, and Control, 121, pp. 566-571.

109. S. Kim, W.W. Clark, and Q.M. Wang, 2005, "Piezoelectric Energy Harvesting with a Clamped Circular Plate: Experimental Study," Journal of Intelligent Material Systems and Structures, 16, pp. 855-864.

110. S. Horowitz, K.A. Kasyap, F. Liu, D. Johnson, T. Nishida, K. Ngo, M. Sheplak, and L. Cattafesta, 2002, "Technology Development for Self-Powered Sensors," Proc. of 1st Flow Control Conference, AIAA-2022-2702.

111. P. Glynne-Jones, S. Beeby, and N.M. White, "Toward a Piezoelectric Vibration-powered Microgenerator," IEEE Proc.-Science, Measurement and Technology, 148, pp. 68-72.

112. Y.B. Jeon, R. Sood, J.H. Jeong, and S.G. Kim, 2005, "MEMS Power Generator with Transverse Mode Thin Film PZT," Sensors and Actuators, 122, pp. 16-22

113. B.S. Lee, J.J. He, W.J. Wu, and W.P. Shih, 2006, "MEMS Generator of Power Harvesting by Vibrations using Piezoelectric Cantilever Beam with Digitate Electrode," Proc. of SPIE, 6169, 121-129.

114. Y.K. Hong and K.S. Moon, 2005, "Single Crystal Piezoelectric Transducers to Harvest Vibration Energy," Proc. of SPIE, 6048, pp. E.1-E.7.

115. H.W. Kim, A. Batra, S. Priya, K. Uchino, D. Markley, R.E. Newnham, and H.F. Hofmann, 2004, "Energy Harvesting Using a Piezoelectric "Cymbal" Transducer in Dynamic Environment," Japanese Journal of Applied Physics, 43, pp. 6178-6183.

116. P.J. Cornwell, J. Goethal, J. Kowko, and M. Damianakis, 2005, "Enhancing Power Harvesting Using a Tuned Auxiliary Structure," Journal of Intelligent Material Systems and Structures, 16, pp. 825-834. 
117. C.B. Williams and R.B. Yates, 1996, "Analysis of a Micro-Electric Generator for Microsystems," Sensors \& Actuators, 52, pp. 8-11.

118. S.C.L. Yuen, J.M.H. Lee, M.H.M, Lee, G.M.H. Chan, F.K. Lei, P.H.W. Leong, W.J. Li, and Y. Yeung, 2004, "AA Size Micro Power Conversion Cell for Wireless Applications," Proc. of the World Congress on Intelligent Control and Automation (WCICA), 6, pp. 5629-5634.

119. P. Glynne-Jones, M.J. Todor, S.P. Beeby, and N.M. White, 2004, "An Electromagnetic, Vibration-Powered Generator for Intelligent Sensor Systems," Sensors \& Actuators, 110, pp. 44-349.

120. M. Mizuno and D.G. Chetwynd, 2003, "Investigation of a Resonance Microgenerator," Journal of Micormechanics and Microengineering, 13, pp. 209-216.

121. N.G. Stephen, 2006 “On Energy Harvesting from Ambient Vibration,” Journal of Sound and Vibration, 293, pp. 409-425.

122. G. Poulin, E. Sarraute, and F. Costa, 2004 "Generation of Electrical Energy for Portable Devices Comparative Study of an Electromagnetic and a Piezoelectric System," Sensors \& Actuators, 116, pp. 61-471.

123. S. Roundy, 2005, “On the Effectiveness of Vibration-based Energy Harvesting," Journal of Intelligent Material Systems and Structures, 16, pp. 09-824.

124. M.E. Staley, A.B. Flatau, 2005, "Characterization of Energy Harvesting Potential of Terfenol-D and Galfenol,” Proc. of SPIE, V.5764, pp. 630-640.

125. http://www.microstrain.com/ last accessed on 01/25/2007.

126. http://perpetuum.co.uk/ last accessed on 01/25/2007.

127. http://www.Ferrosi.com/ last accessed on 01/25/2007.

128. E.E. Lawreence and G.J. Snyder, 2002, "A Study of Heat Sink Performance in Air and Soil for Use in a Thermoelectric Energy Harvesting Device," Proc. of the 21st International Conference on Thermoelectronics, Portland, OR, pp. 446-449.

129. M.D. Rowe, G. Min, S.G. Williams, A. Aoune, K. Matsuura, V.L. Kuznetsov, and L.W. Fu, 1997, "Thermoelectric Recovery of Waste Heat - Case Studies," Proc. of the $32^{\text {nd }}$ Intersociety Energy Conversion Engineering Conference, July 27-Aug 1st, Honolulu, HI, pp. 1075-1079.

130. J. Fleming, W. Ng, and S. Ghamaty, 2004, "Thermoelectric-Based Power System for Unmanned-Air-Vehicle/Microair-Vehicle Applications," Journal of Aircraft, 41, pp. 674-676.

131. U. Birkholz, U. E. Grob, M. Riffel, H. Roth, and U. Stohrer 1988, "Conversion of Waste Exhaust Heat in Automobile Using FeSi2 Thermoelements," Proc. of the 7th International Conference on Thermoelectric Energy Conversion, Arlington, VA, pp. 124-128.

132. K. Matsubara, 2002, "Development of a High Efficient Thermoelectric Stack for a Waste Exhaust Heat Recovery of Vehicles," Proc. of the 21st International Conference on Thermoelectronics, August 25-29th, Portland, OR, pp. 418-423.

133. J.C. Bass, N.B. Elsner, and F.A. Leavitt, 1994, "Performance 1kW Thermoelectric Generator for Diesel Engines," Proc. of AIP Conference, 316, pp. 295-298. 
134. J. Vázquez, M.A. Sanz-Bobi, R. Palacios, and A. Arenas, 2002, "State of the Art of Thermoelectric Generators Based on Heat Recovered from the Exhaust Gases of Automobiles," Proc. of the 7th European Workshop on Thermoelectrics, Pamplona, Spain, Paper No. 17.

135. H.A. Sodano, R. Dereux, G.E. Simmers, and D.J. Inman, 2004, "Power Harvesting Using Thermal Gradients for Recharging Batteries," Proc. of 15th International Conference on Adaptive Structures and Technologies, October 25-27 2004, Bar Harbor, ME.

136. H. Bottner, 2003, “Thermoelectric Micro Devices: Current State, Recent Developments and Future Aspects for Technological Progress and Applications," Proc. of 21st International Conference on Thermoelectric, pp. 511-518.

137. G. J. Snyder, J.R. Lim, CK. Huang, and J.P. Fleurial, 2003, “Thermoelectric Microdevice Fabricated by a MEMS-like Electrochemical Process," NatureMaterials, 2, pp. 528-531.

138. V. Jovanovic and S. Ghamaty, 2006, "Design, Fabrication and Testing of EnergyHarvesting Thermoelectric Generators," Proc. of SPIE, 6173, pp. G.1-G.8.

139. http://www. adsx.com, last accessed on 01/25/2007.

140. H. Guo and A. Lal, 2003, "Nanopower Betavoltaic Microbatteries," Proc. of 12th International Conference on Transducer, Solid-State Sensors, Actuators, and Microsystems, pp. 6-39.

141. W. Sun, N.P. Kherani, K.D. Hirschman, L.L. Gadeken, and P.M. Fauchet, 2005, “A ThreeDimensional Porous Silicon p-n Diode for Betavoltaics and Photovoltaics," Advanced Materials, 17, pp. 1230-1233.

142. http:// www.betabatt.com, last accessed on 01/25/2007.

143. A. Lal and J. Blanchard, 2004, “The Daintiest Dynamos," IEEE Spectrum, pp. 36-41.

144. A. Lal, R. Duggirala, H. Li, 2005, "Pervasive Power: A Radioisotope-Powered Piezoelectric Generator,” IEEE Pervasive Computing, pp. 53-61.

145. D.W. Harrist, 2004, Wireless Battery Charging System Using Radio Frequency Energy Harvesting, M.S. thesis, Department of Electrical Engineering, University of Pittsburgh, Pittsburgh, PA.

146. W.C. Brown, 1996, "The History of Wireless Power Transmission," Solar Energy, 56, pp. 3-21.

147. G.E. Maryniak, 1996, "Status of International Experimentation in Wireless Power transmission," Solar Energy, 56, pp. 87-91.

148. S. Choi, K. Song, W. Golembiewskii, S.H. Chu, G. King, 2004, "Microwave Powers for smart material actuators," Smart Materials and Structures, 13, pp. 38-48.

149. S.D. Briles, D.L. Neagley, D.M. Coates, S.M. Freund, 2004, Remote Down-Hole Well Telemetry, United States Patent \# 6,766,141.

150. B. Strassner, and Chang, K., 2003, "5.8 GHz Circularly Polarized Dual-Rhombic-loop Traveling-wave Rectifying Antenna for Low Poer-Density Wireless Power Transmission Applications," IEEE Transaction on Microwave Theory and Techniques, 51, pp. 1548-1553. 
151. M. Ali, G. Yang, and R. Dougal, 2005, “A new Circularly Polarized Rectenna for Wireless Power Transmission and Data Communication," IEEE Antennas Wireless Propagation, 4, pp. 205-208.

152. Y.J. Ren, K. Chang, 2006, "5.8 GHz Circularly Polarized Dual-Diode Rectenna and Rectenna Array for Microwave Power Transmission," IEEE Transactions on Microwave Theory and Technique, 54, pp. 1495-1502.

153. J. Kim, S.Y. Yang, D.D. Song, S. Jones, S.H. Choi, 2006, "Performance Characterization of Flexible Dipole Rectennas for Smart Actuator Use," Smart Materials and Structures, 15, pp. 809-815.

154. J.Y. Park, S.M. Han, and T. Itoh, 2004, "A Rectenna Design with Harmonic-Rejecting Circular-Sector Antenna," IEEE Antennas and Wireless Propagation Letters, 3, pp. 52-54.

155. C.H. Chin, Q. Xue, C.H. Chan, 2005, "Design of a $5.8 \mathrm{GHz}$ Rectenna Incorporating a New patch Antenna," IEEE Antennas and Wireless Propagation Letters, 4, pp. 175-178.

156. L.W. Epp, A.R. Khan, H.K. Smith, and R.P. Smith, 2000, “A Compact Dual-Polarized 8.51 $\mathrm{GHz}$ Rectenna for High-voltage Actuator Applications," IEEE Transactions on Microwave Theory and Technique, 48, pp. 111-119.

157. J. Zbitou, M. Latrach, S. Toutain, 2006, "Hybrid Rectenna and Monolithic Integrated ZeroBias Microwave Rectifier," IEEE Transactions on Microwave Theory and Technique, 54, pp. 147-152.

158. D.L. Mascarenas, 2006, Development of an Impedance-Based Wireless Sensor Node for Monitoring of Bolted Joint Preload, M.S. Thesis, Dept. of Structural Engineering, University of California, San Diego. (LA-14303-T).

159. G. Park, T.G. Overly, M. Nathnagel, C.R. Farrar, D.L. Mascarenas, M.D. Todd, 2006, “A Wireless Active-Sensor Node for Impedance-Based Structural Health Monitoring," Proc. of USKorea Smart Structures Technology for Steel Structures, Seoul, Korea, pp. 53-62.

160. A. Kasyap, J. Lim, D. Johnson, S. Horowitz, T. Nishida, K. Ngo, M. Sheplak, and L. Cattafesta, 2002, "Energy Reclamation from a Vibrating Piezoceramic Composite Beam," Proc. of 9th International Congress on Sound and Vibration, Orlando, FL, Paper No. 271.

161. G.K. Ottman, H. Hofmann, A.C. Bhatt, and G.A. Lesieutre, 2002, "Adaptive Piezoelectric Energy Harvesting Circuit for Wireless, Remote Power Supply," IEEE Transactions on Power Electronics, 17, pp. 669-676.

162. H. Hofmann, G.K. Ottman, and G.A. Lesieutre, 2002, "Optimized Piezoelectric Energy Circuit Using Step-Down Converter in Discontinuous Conduction Mode," IEEE Transactions on Power Electronics, 18, pp. 696-703.

163. M. Kimura, 1998. Piezoelectric Generation Device, United States Patent Number $5,801,475$.

164. M. Guan, and W.H. Liao, 2006, "On the Energy Storage Devices in Piezoelectric Energy Harvesting," Proc. of SPIE, 6169, pp. C.1-C.9. 
165. N. Elvin, A. Elvin, and D.H. Choi, 2003, "A Self-Powered Damage Detection Sensor," Journal of Strain Analysis, 38, pp. 115-124.

166. E.P. James, M.J. Tudor, S.P. Beeby, N.R. Harris, P. Glynne-Jones, J.N. Ross, and N.M. White, 2004. "An Investigation of Self-Powered Systems for Condition Monitoring Applications," Sensors \& Actuators, 110, pp. 171-176.

167. F.M. Discenzo, D. Chung, and K.A. Loparo, 2006, "Pump Condition Monitoring Using Self-Powered Wireless Sensors," Sound and Vibration, 40 (5), pp. 12-15.

168. K.B. Pfeifer, S.K. Leming, and A.N. Rumpf, 2001, Embedded Self-Powered Micro Sensors for Monitoring the Surety of Critical Buildings and Infrastructures, Sandia National Laboratories report, SAND2001-3619.

169. S. Ha, and F.K. Chang, 2005, "Review of Energy Harvesting Methodologies for Potential SHM Applications," Proc. of 2005 International Workshop on Structural Health Monitoring, pp. 1451-1460.

170. http://www.cymbet.com/, last accessed on 01/25/2007.

171. http://www.itnes.com/, last accessed on 01/25/2007. 


\section{WORKSHOP PARTICIPANTS}

\begin{tabular}{|c|c|}
\hline Name & Affiliation \\
\hline Avitabile, Peter & University of Massachusetts \\
\hline Ayers, James & Oregon State University \\
\hline Chalermek, Intanagonwiwat & University of California, San Diego \\
\hline Clark, William & University of Pittsburgh \\
\hline Cornwell, Phillip & Rose-Hulman Institute of Technology \\
\hline Drewniak, James & University of Missouri, Rolla \\
\hline Elvin, Niell & Michigan State University \\
\hline Flatau, Alison & University of Maryland, College Park \\
\hline Ganley, Jeffrey & Air Force Weapons Laboratory \\
\hline Hodgkiss, Bill & University of California, San Diego \\
\hline Kamyabi, Bozorcmehr & University of California, San Diego \\
\hline Le, Triet & Oregon State University \\
\hline Masquelier, Michael & Motorola, Inc. \\
\hline Mossi, Karla & Virginia Commonwealth University \\
\hline Niezrecki, Christopher & University of Massachusetts, Lowell \\
\hline Nishida, Toshikazu & University of Florida \\
\hline Peairs, Daniel & Virginia Tech \\
\hline Sanders, Brian & Air Force Office of Scientific Research \\
\hline Sang-Gook, Kim & MIT \\
\hline Sazonvo, Edward & Clarkson University \\
\hline Rosing, Tajana & University of California, San Diego \\
\hline Sanders, Brian & AFRL \\
\hline Sodano, Henry & Michigan Technological University \\
\hline Sumali, Hartono (Anton) & Sandia National Laboratories \\
\hline Todd, Michael & University of California, San Diego \\
\hline Bement, Matthew & LANL/INST-OFF \\
\hline Dreicer, Jared & LANL/PADSTE \\
\hline Hundhausen, Jason & LANL/WT-7 \\
\hline Lashley, Jason C. & LANL/MST-6 \\
\hline Mielke, Angela & LANL/ISR-3 \\
\hline Nadler, Brett & LANL/WT-4 \\
\hline Park, Gyuhae & LANL/INST-OFF \\
\hline Puckett, Anthony & LANL/WT-2 \\
\hline Rutherford, Mandy & LANL/WT-2 \\
\hline Smith, Jim & LANL/MST-6 \\
\hline Swift, Greg & LANL/MPA-10 \\
\hline Thien, Andrew & LANL/WT-2 \\
\hline
\end{tabular}




\section{DISTRIBUTION}

Los Alamos National Laboratory

Sami Ayyorgun MS B-287

Carl Beard, MS E585

Doug Beason, MS A135

John Benner, MS A115

Steve Black, MS C926

Mike Burns, MS A135

Scott Doebling, MS T-080

Allen Graham, MS T-011

Scott Gibbs, MS C-921

Gary Grider, MS B-272

Francois Hemez, MS F-699

Nick Hengartner, MS M-997

Paul Johnson, MS D-443

Kevin Jones, MS H-809

Brett Knapp, MS A-109

Mike Mallory, MS E-585

Glen Mara, MS A-107

Ryan Maupin, MS T-080

Charles McMillan, MS A-113

Albert Migliori, MS E-536

Bill Priedhorsky, MS A-135

Ed Rodríguez, MS P-946

Nancy Sauer, MS T-001

John Schultze, MS P-001

David Sharp, MS B-213

Denise Liechty, MS D-471

Terry Wallace, MS A-127

Paul Wantuck, MS C-921

David Watkins MS M-001 


\section{External}

Doug Adams

Purdue University

School of Mechanical Engineering,

West Lafayette, IN 47907-1077

Tom Baca

Structural Dynamics Engineering Department MS 0557

Sandia National Laboratory

Albuquerque, NM 87185-5800

Tom Carne

Dept. 2741

Sandia National Laboratory

Albuquerque, NM 87185-5800

Fu-Kuo Chang

Dept. of Aeronautics and Astronautics

Stanford University

Stanford, CA 94305

KyuZong Cho

Dept. of Mechanical Engineering

Chonnam National University

Kwangju, Korea, 500-757

Ahmed Elgamal

University of California, San Diego

9500 Gilman Drive \# 0085

La Jolla, CA 92093-0085

Dave Ewins

Imperial College

Mechanical Engineering Dept.

Exhibition Road

London SW7 2BX, UK

Lothar Gaul

Institut A fur Mechanik

Universitat Stuttgart

Pfaffenwaldring 9

70550 Stuttgart, Germany

Spilios D. Fassois

Stochastic Mechanical Systems (SMS)

Department of Mechanical and

Aeronautical Engineering

University of Patras

26500 Patras, Greece
Gregory Fenves

Department of Civil and Environmental Engineering, MC 1710

University of California

Berkeley, CA 94720-1710

Mike Friswell

Dept. of Mechanical Engineering

University of Wales, Swansea

Singleton Park,

Swansea, SA2 8PP

UK

Claus-Peter Fritzen

Institute of Mechanics and Automatic Control

University of Siegen

Paul-Bonatz-Str. 9-11

D-57068 Siegen, Germany

Victor Giurgiutiu

Air Force Office of Scientific Research

875 North Randolph Street, Suite 325

Arlington, VA 22203

Jean-Claude Golinval

Université de Liège

Département d'Aérospatiale, Mécanique

et Matériaux

Institut de Mécanique et Génie Civil

1, Chemin des Chevreuils, B52/3

4000 Liège, Belgium

Alfredo Guemes

UPM

ETSI Aeronautics

Madrid, 28016

Spain

Dan Inman

Virginia Polytechnic Institute of State Univ.

310 New Engineering Building

Mail code 0261

Blacksburg, VA 24061-0219

Kumar Jata

AFRL

$223010^{\text {th }}$ Street

Wright-Patterson Air Force Base, OH 45433 
Daniel Kammer

University of Wisconsin-Madison

Dept. of Engr. Mech. and Astronautics

3352 Engr. Hall, 1415 Johnson Drive

Madison, WI 53706

Tom Kenny

Stanford University,

Department of Mechanical Engineering, Terman 540,

MC 4021,

Stanford, CA 94305-4021

Anne Kiremidjian

Department of Engineering

Stanford University

Terman Engineering Center 238

Stanford, CA 94305-4020

Jim Larson

Air Force Research Laboratory, AFRL/MLLMN

Wright-Patterson Air Force Base, OH 45433

Kincho Law

Dept. of Civil Engineering

Stanford University

Stanford, CA 94305-4020

Les Lee

Air Force Office of Scientific Research

875 North Randolph Street, Suite 325

Arlington, VA 22203

Don Leo

DARPA

3701 North Fairfax Drive

Arlington, VA 22203-1714

Nick Lieven

Dept. of Aerospace Engineering

University of Bristol, Queen's Bldg.

Bristol, BS8 1TR, UK

Paul Linden

University of California, San Diego

9500 Gilman Drive \# 0411

La Jolla, CA 92093-0411

Shih-Chi Liu

National Science Foundation

4201 Wilson Blvd.

Arlington, VA 22230
Jerome Lynch

Department of Civil and Environmental Engineering 2328 G. G. Brown Building

University of Michigan, Ann Arbor

Ann Arbor, MI 48109-2125

Keith Marzullo

University of California, San Diego

Department of Computer Science and Engineering

EBU 3B 3126

La Jolla, CA 92093-0404

Sami Masri

University of Southern California

Department of Civil Engineering

MC 2531

Los Angeles, CA 90089-2531

Randy Mayes

Sandia National Laboratory

MS0557

PO Box 5800

Albuquerque, NM 87185

David McCallen

Center for Complex Distributed Systems

Lawrence Livermore National Laboratory

Livermore, CA 94550

Akira Mita

Graduate School of Science and Technology, Keio

University

8-14-1 Hiyoshi, Kohoku-ku

Yokohama 223-8522

Japan

Richard Pappa

NASA Langley Research Center

MS 230

Hampton, VA 23681

Michael Pecht

1103 Engineering Laboratory Bldg

University of Maryland,

College Park, MD 20742-3035

Charlie Pickrel

Structural Dynamics Laboratory

Boeing Commercial Airplanes Group

Mail Code 1W-06, P.O. Box 3707

Seattle, WA 98124-2207 
Darryll J. Pines

Dept. of Aerospace Engineering

Rm 3154 Engineering Classroom Bldg

University of Maryland

College Park, MD 20742

Ramesh Rao

University of California, San Diego

9500 Gilman Dr.

La Jolla, CA 92093-0436

Mark Schulz

Department of Mechanical and Nuclear Engineering

University of Cincinnati

Cincinnati, OH 45221-0072

Frieder Seible

University of California, San Diego

9500 Gilman Dr. \#0403

La Jolla, CA 92093-0403

Bill Spencer

Department of Civil and Environmental Engineering 205 North Mathews Ave

University of Illinois, Urbana-Champaign

Urbana, IL 61801-2352

Brain Wardle

Department of Aeronautics and Astronautics

MIT

77 Massachusetts Avenue, 33-314

Cambridge, MA 02139

Ming Wang

Dept. of Civil and Material Engineering (M/C 246)

842 West Taylor St.

Chicago, Illinois 60607-7023
Ed White

Boeing Corp.

P.O. Box 516

St. Louis, MO 63166

Keith Worden

The University of Sheffield

Department of Mechanical and Process Engineering PO Box 600

Mappin St

Sheffield, S1 3JD UK

Paul Wright

Department of Mechanical Engineering

5133 Etcheverry Hall, Mailstop 1740

University of California, Berkeley

Berkeley, CA 94720-1740

Paul Yu

University of California, San Diego

9500 Gilman Dr. \#0407

La Jolla, CA 92093-0407

Chung-Bang Yun

Dept. of Civil Engineering

Korean Institute of Advanced Science and

Technology

373-1, Kusong-dong, Yusong-gu,

Taejon, KOREA, 305-701

Dave Zimmerman

University of Houston

Department of Mechanical Engineering

Houston, TX 77204-4792 

This report has been reproduced directly from the best available copy. It is available electronically on the Web (http://www.doe.gov/bridge).

Copies are available for sale to U.S. Department of Energy employees and contractors from:

Office of Scientific and Technical Information

P.O. Box 62

Oak Ridge, TN 37831

(865) 576-8401

Copies are available for sale to the public from: National Technical Information Service

U.S. Department of Commerce

5285 Port Royal Road

Springfield, VA 22161

(800) 553-6847 
- Los Alamos NATIONAL LABORATORY

EST.1943 